TRANSACTIONS OF THE

AMERICAN MATHEMATICAL SOCIETY

Volume 349, Number 9, September 1997, Pages 3409-3468

S $0002-9947(97) 01845-X$

\title{
ELLIPTIC THREE-FOLDS II: MULTIPLE FIBRES
}

\author{
MARK GROSS
}

\begin{abstract}
Let $f: X \rightarrow S$ be an elliptic fibration with a section, where $S$ is a projective surface and $X$ is a projective threefold. We determine when it is possible to perform a logarithmic transformation along a closed subset $Z \subseteq S$ to obtain a new elliptic fibration $f^{\prime}: X^{\prime} \rightarrow S$ which now has multiple fibres along $Z$. This is done in the setting of Ogg-Shafarevich theory. We find a number of obstructions to performing such a logarithmic transformation, the very last of which takes values in the torsion part of the codimension 2 Chow group of $X$.
\end{abstract}

\section{INTRODUCTION}

Suppose that we would like to classify algebraic elliptic three-folds, i.e. algebraic three-folds $X$ with a fibration $f: X \rightarrow S$ whose general fibre is an elliptic curve. In analogy with the classification of elliptic surfaces, there are three steps:

(1) Classify elliptic fibrations with section. These can all be obtained birationally using Weierstrass models.

(2) Classify elliptic fibrations $f^{\prime}: X \rightarrow S$ without multiple fibres which have a given jacobian fibration $f: J \rightarrow S$. This has already been carried out in [8]; the set of such elliptic fibrations is parametrized up to birational equivalence by the Tate-Shafarevich group $\amalg_{S}(A)$, where $A$ is the generic fibre of $f$.

(3) Understand how to perform logarithmic transformations. In the case of complex elliptic surfaces, a logarithmic transformation is an operation for producing multiple fibres over points in the base curve. It is a purely local operation, which can then be patched to give a new complex elliptic surface, which may or may not be algebraic. However, it is well understood when the resulting surface will be algebraic. In the case of threefolds, this is a considerably more complicated task. Now one should expect to perform logarithmic transformations along curves in the base surface, and so when dealing with a global base, one cannot expect a purely local description for a logarithmic transformation.

It is step 3 which this paper carries out. Instead of the local analytic description of logarithmic transformations given by Kodaira in [14] and extended to higher dimensions by N. Nakayama in [19], we take the algebraic approach of Ogg-Shafarevich theory. (See [20] and [21], and also [4].) In this point of view,

Received by the editors June 19, 1995.

1991 Mathematics Subject Classification. Primary 14J30.

This material is based upon work supported by the North Atlantic Treaty Organization under a Grant awarded in 1990. Research at MSRI supported in part by NSF grant \#DMS 9022140.

(C)1997 American Mathematical Society 
we begin with a jacobian elliptic fibration $f: J \rightarrow S$ with generic fibre $A$, and we compute $\amalg_{S-Z}(A)$, which is essentially the set of elliptic fibrations with multiple fibres only over $Z$.

More explicitly, as explained in $\S 1$, we can always assume $J$ and $S$ are nonsingular, and $f: J \rightarrow S$ is flat with discriminant locus $\Sigma$ such that the one dimensional component of $Z \cup \Sigma_{\text {red }}$ is a simple normal crossings (s.n.c.) divisor. This can be accomplished using [16] by blowing up the base, if necessary. If $A$ is the generic fibre of $f$, we can think of $A$ as an étale sheaf on $\eta$. If $i: \eta \rightarrow S$ is the inclusion of the generic point of $\eta$ in $S$, then $\amalg_{S}(A)=H^{1}\left(S, i_{*} A\right)$ (étale cohomology) is the Tate-Shafarevich group which essentially classifies, in this case, fibrations over $S$ with Jacobian $J \rightarrow S$ which have no multiple fibres. More precisely, it was shown in [8] that $\amalg_{S}(A)$ is the set of isomorphism classes $E$ of curves of genus one over $\eta$ with jacobian $A$, such that there exists a model $f^{\prime}: X \rightarrow S$ with $f^{\prime}$ relatively minimal in the sense of Mori with $X_{\eta}=E$ and $f^{\prime}: X \rightarrow S$ having only so-called locally trivial multiple fibres, i.e. multiple fibres for which there exists locally a rational section of the fibration. Such multiple fibres are necessarily isolated, and can only occur at very special places; for the most part, we ignore them. Thus, what we wish to calculate is the quotient

$$
\amalg_{S-Z}(A) / \amalg_{S}(A) .
$$

This will tell us which new fibrations can occur when we allow multiple fibres over $Z$. To do this, we use the exact sequence of local cohomology:

$$
0 \rightarrow H^{1}\left(S, i_{*} A\right) \rightarrow H^{1}\left(S-Z, i_{*} A\right) \rightarrow H_{Z}^{2}\left(S, i_{*} A\right) \rightarrow H^{2}\left(S, i_{*} A\right) .
$$

We first calculate $H_{Z}^{2}\left(S, i_{*} A\right)$ and obtain a group of possible global invariants. The obstruction to realising these global invariants lives in $H^{2}\left(S, i_{*} A\right)$. For example, if $Z$ is a smooth curve such that $f^{-1}(Z)$ is also smooth, then $H_{Z}^{2}\left(S, i_{*} A\right)$ is the torsion part of the Mordell-Weil group of the elliptic fibration $f^{-1}(Z) \rightarrow Z$. The situation becomes more complicated when $Z$ contains components of $\Sigma$, but in this case it is essentially a local calculation. We complete these calculations in $\S 2$.

The next problem is the obstruction in $H^{2}\left(S, i_{*} A\right)$. Without going into details now, there is an exact sequence

$$
0 \rightarrow G_{1} \rightarrow H^{2}\left(S, i_{*} A\right) \rightarrow G_{2}
$$

for certain groups $G_{1}$ and $G_{2}$ which can be calculated. The obstruction in $G_{2}$, which we call the first obstruction, is essentially discrete and can be completely calculated. Intuitively, this obstruction detects the following problem. If, say, $Z$ is a smooth curve which intersects some component $\Sigma$ only once, say a component $\Sigma_{1}$ for which $f^{-1}\left(\Sigma_{1}\right)$ consists of several components, we would expect that having multiple fibres along $Z$ would force us to rearrange those components in such a way that there is now some ramification in the map of these components to $\Sigma_{1}$ possibly violating Hurwitz's formula. This is not a precise statement, but gives the flavor of the first obstruction.

The obstruction in $G_{1}$ is more subtle. Given an element of $H_{Z}^{2}\left(S, i_{*} A\right)$ whose first obstruction is zero, one can construct a torsion codimension 2 algebraic cycle on $X$ corresponding to it. We show, using the Bloch map for torsion algebraic cycles, that this element of $H_{Z}^{2}\left(S, i_{*} A\right)$ can be realised only if the correspond- 
ing torsion cycle is rationally equivalent to zero, modulo a set of cycles contained in fibres of $f$. In fact, this obstruction in a sense has already appeared in the case that $S$ is a curve, except that in that case, the obstruction turns out to be always zero unless $J \rightarrow S$ is trivial, and thus any logarithmic transformation on a non-trivial fibration is algebraic. This is not the case once $S$ is a surface.

The calculations are all performed using local cohomology calculations on $J$ and $S$. In order to do this, we need very explicit descriptions of the geometry of $J$. For this, we rely heavily on the explicit resolutions of Weierstrass models given by Miranda in [16]. Without these explicit resolutions, there appears to be no way to obtain useful global information. One unpleasant side effect of the need to use such explicit resolutions is that some of the computations for $I_{M}$ fibres, $M \geq 1$, become very long. We leave many of these details to an appendix.

I would like to stress that the final results can be applied without using étale cohomology, the information that one needs being completely geometric. To calculate $H_{Z}^{2}\left(S, i_{*} A\right)$ one only needs to know the torsion part of certain Mordell-Weil groups of elliptic surfaces, as mentioned above, and a description of the discriminant locus of the elliptic fibration in question. To calculate the first obstruction one needs no additional information. The last obstruction requires information about rational equivalence on $J$. In many practical applications of these results, one can determine everything one needs to know. Examples are given throughout the text, and the reader can consult [12] for further examples.

The paper is organized as follows. $\S 1$ reviews basic results on models of elliptic fibrations and the Tate-Shafarevich groups. It closes with a number of basic computations of local cohomology which will be used throughout the paper. $\S 2$ then computes $H_{Z}^{2}\left(S, i_{*} A\right)$, and $\S \S 3$ and 4 detail the first and second obstructions, respectively. The appendix contains details about type $I_{M}$ fibres, $M \geq 1$.

There are two works which have considered this problem in more restricted circumstances. [9] considers the problem of logarithmic transformations if the elliptic fibration is the product of the base with an elliptic curve, but also constructs nonalgebraic examples. As already mentioned, [19] considers these questions locally, in all dimensions. Nakayama manages to treat all dimensions by relying on monodromy arguments rather than by using a model for the original elliptic fibration as we do. As a result, his methods do not extend to the global situation. We reproduce some of his results in dimension 3 using our own techniques to save some effort in the global calculations.

Finally, I would like to mention one important application of these results to the classification of three-folds. In [12], the results of this paper play a crucial role in giving a theoretical classification of elliptic Calabi-Yau manifolds. There all aspects of the current work are used in order to show, up to birational equivalence, that there are only a finite number of families of elliptic Calabi-Yau three-folds. Since this paper has been revised since [12] has appeared in print, I would like to note how some references to this paper have changed. References to $\S 4$ should now be references to $\S 3$, and references to $\S 5$ should now be references to $\S 4$. The reference to Theorem 4.5 should now be to Theorem 3.4, and the reference to Example 5.2 should now be to Theorem 4.3 or Example 4.5.

Much of this work was done while visiting Université de Paris VI; I would like to thank C. Peskine for his hospitality there. I also thank I. Dolgachev and A. Grassi for useful conversations concerning this work. 


\section{Preliminaries}

We adopt the following notation:

$S$ : a normal integral excellent scheme. In this paper $S$ will always be a variety over an algebraically closed field $k$ of characteristic 0 , or an open subscheme of the spectrum of the strict henselization of a local ring of such a variety.

$S^{(n)}$ : the set of points in $S$ of codimension $n$, i.e. such that $\operatorname{dim} \mathcal{O}_{S, s}=n$.

$\eta$ : the generic point of $S$.

$i: \eta \rightarrow S$ : the natural inclusion morphism.

$K=K(\eta)$ : the field of rational functions on $S$.

$A$ : a fixed abelian variety of dimension 1 over $K$.

$\mathcal{O}_{S, \bar{s}}$ : the strict henselization of the local ring $\mathcal{O}_{S, s}$.

$K_{\bar{s}}$ : the field of fractions of $\mathcal{O}_{S, \bar{s}}$.

$\eta_{\bar{s}}: \operatorname{Spec} K_{\bar{s}}$

$A_{\bar{s}}=A \times_{\eta} \eta_{\bar{s}}$.

$k(s)$ : the residue field of a point $s \in S$.

${ }_{n} G$ : the elements of an abelian group $G$ killed by multiplication by $n$.

All cohomology is étale unless otherwise stated.

We first recall some results from [8] on Ogg-Shafarevich theory for higher dimensional varieties. See [8] for details. We recall that given an elliptic curve $A$ over a field $K$ (in our case the function field of $S$ ), we put $W C(A)=H^{1}(\operatorname{Spec} K, A)$, which can be identified with the set of isomorphism classes of curves of genus one over $K$ which have $A$ as their jacobian. Here we think of $A$ as defining an étale sheaf over $\operatorname{Spec} K$.

If $s \in S$, there is a natural localization map

$$
W C(A) \rightarrow W C\left(A_{\bar{s}}\right)
$$

given by $E \mapsto E \times_{\eta} \eta_{\bar{s}}$, and we define $\amalg_{S}(A)=\operatorname{ker}\left(W C(A) \rightarrow \prod_{s \in S} W C\left(A_{\bar{s}}\right)\right)$. The cohomological interpretation is $\amalg_{S}(A)=H^{1}\left(S, i_{*} A\right)$. We recall how to calculate $\amalg_{S}(A)$ given the following hypothesis:

Hypothesis. There exists a flat proper fibration $f: X \rightarrow S$ with generic fibre equal to $A$, with $X$ and $S$ non-singular, and $f$ has a regular section $\sigma_{0}: S \rightarrow X$.

Then we have

Theorem 1.1. Let $P_{X / S}=R^{1} f_{*} \mathbf{G}_{m}$. Then there is an exact sequence

$$
0 \rightarrow \mathcal{E} \rightarrow P_{X / S} \rightarrow i_{*} i^{*} P_{X / S} \rightarrow 0
$$

such that $\mathcal{E}$ has support contained in the set

$$
\left\{s \in S \mid X_{\bar{s}} \text { is reducible }\right\} .
$$

Furthermore,

$$
\begin{gathered}
H^{i}\left(S, P_{X / S}\right)=H^{i+1}\left(X, \mathbf{G}_{m}\right) / H^{i+1}\left(S, \mathbf{G}_{m}\right), \\
H^{1}(S, \mathcal{E})=0, \quad H^{1}\left(S, i_{*} i^{*} P_{X / S}\right)=H^{1}\left(S, i_{*} A\right),
\end{gathered}
$$

and there is an exact sequence

$$
0 \rightarrow H^{2}\left(S, i_{*} A\right) \rightarrow H^{2}\left(S, i_{*} i^{*} P_{X / S}\right) \rightarrow H^{1}(S, \mathbf{Q} / \mathbf{Z})
$$


coming from the exact sequence

$$
0 \rightarrow i_{*} A \rightarrow i_{*} i^{*} P_{X / S} \rightarrow \mathbf{Z} \rightarrow 0 .
$$

This yields an exact sequence

$$
\begin{aligned}
0 \rightarrow \frac{H^{2}\left(X, \mathbf{G}_{m}\right)}{H^{2}\left(S, \mathbf{G}_{m}\right)} \rightarrow \amalg_{S}(A) \rightarrow H^{2}(S, \mathcal{E}) \rightarrow \frac{H^{3}\left(X, \mathbf{G}_{m}\right)}{H^{3}\left(S, \mathbf{G}_{m}\right)} \\
\quad \rightarrow H^{2}\left(S, i_{*} i^{*} P_{X / S}\right) \rightarrow H^{3}(S, \mathcal{E}) .
\end{aligned}
$$

Furthermore, if each reduced component of the discriminant locus $\Sigma$ of $f$ is normal, then

$$
H^{i}(S, \mathcal{E})=\prod_{t \in S^{(1)}} \operatorname{coker}\left(H^{i-1}(C(t), \mathbf{Q} / \mathbf{Z}) \rightarrow H^{i-1}(\bar{C}(t), \mathbf{Q} / \mathbf{Z})\right), \quad i \geq 2 .
$$

Here, $C(t)=\{t\}^{-}$. To define $\bar{C}(t)$, let $X_{t}^{i}$ be the components of the fibre $X_{t}, \tilde{X}_{t}^{i}$ the normalization of $X_{t}^{i}$, and $\tilde{X}_{t}^{i} \rightarrow t_{i} \rightarrow t$ the Stein factorization. Let $C\left(t_{i}\right)$ be the normalization of $C(t)$ in $t_{i}$, and set $\bar{C}(t)$ to be the disjoint union of the $C\left(t_{i}\right)$.

Proof. [8] , §1.

We recall some definitions from [8] :

Definition. A projective morphism $f: X \rightarrow S$ is called an elliptic fibration if its generic fibre is a smooth elliptic curve and all fibres are geometrically connected. The closed subset

$$
\Sigma=\left\{s \in S \mid X_{s} \text { is not regular }\right\}
$$

is called the discriminant locus. If $t \in S^{(1)}$, the fibre type of $t$ is the Kodaira fibre type $\left({ }_{m} I_{n}, I_{n}^{*}, I I, I I^{*}, I I I, I I I^{*}, I V, I V^{*}\right)$ of the central fibre of a relative minimal model (or Néron model) of $X(\bar{t})=X \times{ }_{S} \operatorname{Spec} \mathcal{O}_{S, \bar{t}}$. (See [1] , page 150, for a description of these singular fibres.) A collision is a singular point of $\Sigma$. The closed subset

$$
\Sigma^{m}=\left\{s \in S \mid f \text { is not smooth for any } x \in f^{-1}(s)\right\}
$$

is called the multiple locus of $f$. A fibre over a point $s \in \Sigma^{m}$ is called multiple. A fibre is called an isolated multiple fibre if it is over a zero-dimensional component of $\Sigma^{m}$. A section (resp. a birational section) of $f$ is a closed subscheme $Y$ of $X$ for which the restriction of $f$ to $Y$ is an isomorphism (resp. a birational isomorphism).

Next we review the definition of a Weierstrass model. See [7], [17], [18] for details. Let $\mathcal{L}$ be a line bundle on a scheme $S, a \in H^{0}\left(S, \mathcal{L}^{\otimes 4}\right)$, and $b \in H^{0}\left(S, \mathcal{L}^{\otimes 6}\right)$ such that $4 a^{3}+27 b^{2}$ is a non-zero section of $\mathcal{L}^{\otimes 12}$. Let $\mathbf{P}=\mathbf{P}\left(\mathcal{O}_{S} \oplus \mathcal{L}^{\otimes-2} \oplus \mathcal{L}^{\otimes-3}\right)$, $\pi: \mathbf{P} \rightarrow S$ the natural projection, and $\mathcal{O}_{\mathbf{P}}(1)$ be the tautological line bundle on $\mathbf{P}$. We define the scheme $W(\mathcal{L}, a, b)$ as a closed subscheme of $\mathbf{P}$ given by the equation $Y^{2} Z=X^{3}+a X Z^{2}+b Z^{3}$ where $X, Y$ and $Z$ are given by the sections of $\mathcal{O}_{\mathbf{P}}(1) \otimes \mathcal{L}^{\otimes 2}, \mathcal{O}_{\mathbf{P}}(1) \otimes \mathcal{L}^{\otimes 3}$, and $\mathcal{O}_{\mathbf{P}}(1)$ which correspond to the natural injections of $\mathcal{L}^{\otimes-2}, \mathcal{L}^{\otimes-3}$ and $\mathcal{O}_{S}$ into $\pi_{*} \mathcal{O}_{\mathbf{P}}(1)=\mathcal{O}_{S} \oplus \mathcal{L}^{\otimes-2} \oplus \mathcal{L}^{\otimes-3}$, respectively.

The structure morphism $W(\mathcal{L}, a, b) \rightarrow S$ is a flat elliptic fibration, called a Weierstrass fibration. It has a section $\sigma_{0}: S \rightarrow W(\mathcal{L}, a, b)$ defined by the $S$ point $(X, Y, Z)=(0,1,0)$. It is easy to see that $\sigma_{0}(S)$ lies in the smooth locus of $W(\mathcal{L}, a, b)$. We will call this section the section at infinity. Its conormal bundle is isomorphic to $\mathcal{L}$. 
The discriminant locus of $W(\mathcal{L}, a, b) \rightarrow S$ is equal to the support of the Cartier divisor defined by the section $\Sigma$ of $\mathcal{L}^{\otimes 12}$ given by $4 a^{3}+27 b^{2}$. This gives the discriminant locus a scheme structure. One can define the $J$-invariant $J$ of $W(\mathcal{L}, a, b)$ as a section of $\mathbf{P}^{1} S$ given by $J(s)=4 a^{3} /\left(4 a^{3}+27 b^{2}\right)$. If $a$ and $b$ do not have any common zeroes, then $J$ defines a morphism $J: S \rightarrow \mathbf{P}^{1}$. This is true in particular if the reduced discriminant locus $\Sigma_{r e d}$ is s.n.c.

For an interpretation of $\amalg_{S}(A)$, we restrict to $\operatorname{dim} X=3$, char $k=0$, in which case we restrict to a special sort of model, a Miranda model. This is obtained by blowing up the base $S$ until only certain sorts of collisions occur, in which case [16] gives an explicit flat resolution of the Weierstrass model.

Definition. A Miranda elliptic fibration is an elliptic fibration $f: X \rightarrow S$ such that

a) $X$ and $S$ are regular and $f$ is flat and has a regular section;

b) the discriminant locus $\Sigma_{\text {red }}$ has simple normal crossing;

c) All collisions are of type $I_{M_{1}}+I_{M_{2}}, I_{M_{1}}+I_{M_{2}}^{*}, I I+I V, I I+I_{0}^{*}, I I+I V^{*}$, $I V+I_{0}^{*}$ or $I I I+I_{0}^{*}$.

Theorem 1.2. Let $f: X \rightarrow S$ be a Miranda model, and $E \in \amalg_{S}(A)$. Then if $f^{\prime}: X^{\prime} \rightarrow S$ is a relatively minimal model (in the sense of Mori) with $X_{\eta}^{\prime}=E$, then $f^{\prime}$ has no multiple fibres, except possibly over collisions of type $I V+I_{0}^{*}$.

Proof. This is a part of Theorem 2.21 and Remark 2.22 of [8] .

For our purposes, it will be useful to modify this model slightly. First, as noted in [8], we have to avoid collisions of type $I_{M_{1}}+I_{M_{2}}$ where both $M_{1}$ and $M_{2}$ are odd, as these may not have a small resolution in the category of schemes. This may be done by blowing up the base. We also note that Miranda's resolution is obtained by resolving the singularities of the Weierstrass model. In resolving a collision of type $I_{M_{1}}+I_{M_{2}}$, where one has fibre type $I_{M_{1}}$ over one branch of $\Sigma$ meeting another branch of $\Sigma$ with fibre type $I_{M_{2}}$, one first blows up the singular curves over one branch, and then over the other branch, and one finds one has a non-singular model. The order, however, will affect the final result. We will assume that we resolve the curve with fibre type $I_{M}, M$ even, before any curve of fibre type $I_{M}, M$ odd. This means we have only one sort of resolution to worry about, and the details will be given in Table 1, at the end of the paper.

Another problem arises when $M$ is odd. If $C \subseteq S$ is a curve of fibre type $I_{M}$, $M=1$, then $f^{-1}(C)$ definitely is not normal; if $M>1$, some component of $f^{-1}(C)$ may not be normal. Indeed, the Miranda model is constructed as a double cover, and a fibre of type $I_{M}$ is a double cover of the dotted lines in Figure 1.1 if $M$ is even, where the solid lines represent the branch locus; and in Figure 1.2 if $M$ is odd (or in Figure 1.3 if $M=1$ ). In the odd case, the last component may or may not split into two surfaces over $C$; if it does not split, that component will not be normal, the singular locus being traced out by the point $P$. In any case, we construct a modified Miranda model by blowing up all curves traced out by the point $P$; this will remove any singularities, and $f^{-1}(C)$ becomes a s.n.c. divisor. We do this over all curves $C$ with $I_{M}$ odd, so that we can treat the odd case uniformly. Table 1 gives information about these modified models. Notice the modified model is still flat, so we can still apply Theorem 1.1.

The above discussion also shows that there are two cases if $C$ is a curve of type $I_{M}: f^{-1}(C)$ may split into $M$ components, or into $\lfloor M / 2\rfloor+1$ components (before 


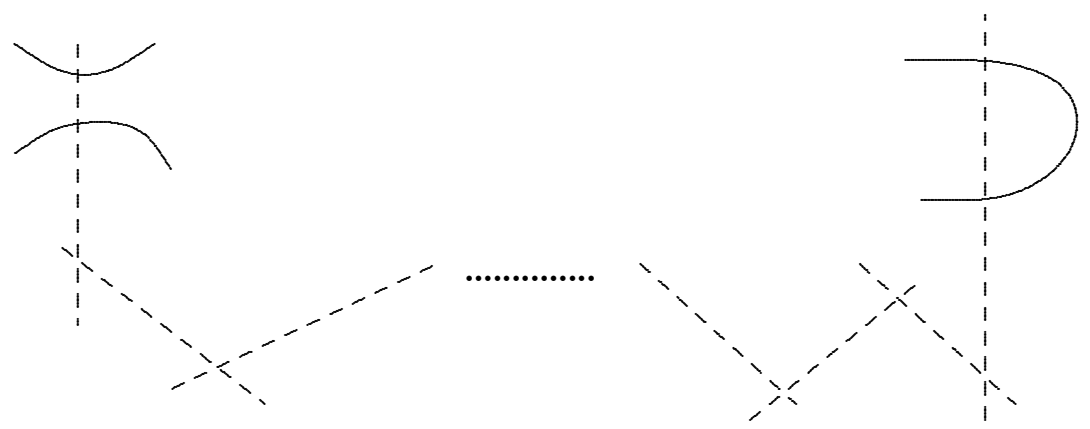

FiguRe 1.1

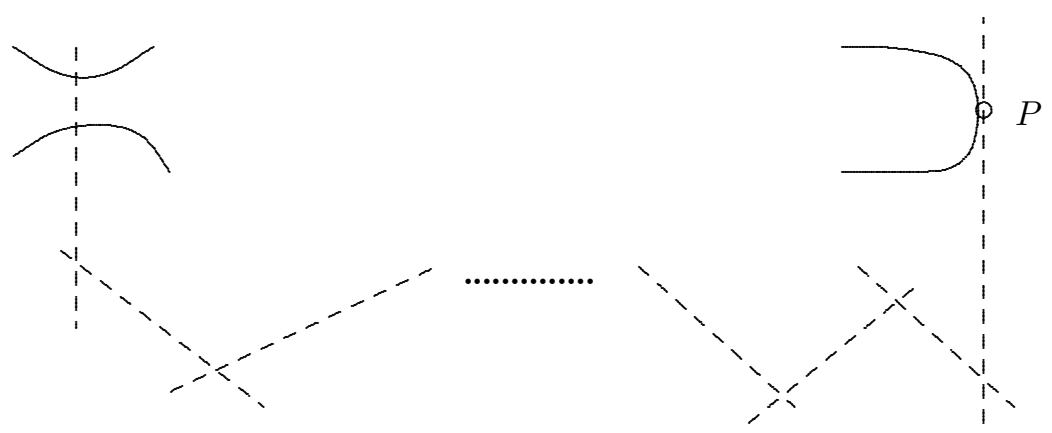

FiguRe 1.2

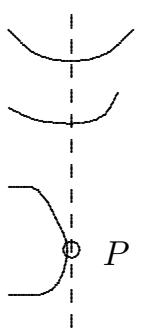

FiguRe 1.3

blowing up in the odd case). We refer to these as case $I$ and case $I^{*}$ respectively. After blowing up, we can describe these two cases as follows, putting

$$
M^{\prime}= \begin{cases}M & \text { if } M \text { is even } \\ M+1 & \text { if } M \text { is odd }\end{cases}
$$

Case I. $f^{-1}(C)$ splits into $M^{\prime}$ components, $Y_{1}, \ldots, Y_{M^{\prime}}$, each a ruled surface over $C, Y_{1}$ intersecting the zero-section of $f: X \rightarrow S$. In addition, $Y_{1} \cap \operatorname{Sing}\left(f^{-1}(C)\right)$ splits into two curves.

Case I*. $f^{-1}(C)$ splits into $M^{\prime} / 2+1$ components, $Y_{1}, \ldots, Y_{M^{\prime} / 2+1}$, with $Y_{1}$ and $Y_{M^{\prime} / 2+1}$ ruled surfaces over $C$ and $Y_{2}, \ldots, Y_{M^{\prime} / 2}$ ruled surfaces over a double cover 
of $C$. In addition, $Y_{1} \cap \operatorname{Sing}\left(f^{-1}(C)\right)$ is irreducible. (When $M=1$ or 2 , this is the only way to distinguish between Case $I$ and $I^{*}$. For fibre type $I_{1}$, the only way to distinguish between these two cases is to perform the blowing up to arrive at the modified Miranda model. We shall see that these two cases present very different behavior when trying to make logarithmic transformations along $C$.)

Finally, we note that if the curve $C$ intersects a curve of fibre type $I_{N}^{*}$ for some $N$, then it is necessarily of case $I^{*}$, as can be seen from Miranda's description of $I_{M}+I_{N}^{*}$ collisions ([16] or Table 2). Thus the notation $I$ and $I^{*}$.

Thus we will adopt the following for the remainder of the paper:

Additional Hypothesis. $f: X \rightarrow S$ is a modified Miranda model, $\operatorname{dim} X=3$, char $k=0$, and $Z \subseteq S$ is a closed set such that $Z \cup \Sigma_{\text {red }}$ is a simple normal crossing divisor plus a finite set of points.

Our goal is to calculate

$$
\frac{\amalg_{S-Z}(A)}{\amalg_{S}(A)} .
$$

Note that the assumption that $Z \cup \Sigma_{\text {red }}$ is s.n.c. is not problematic. Indeed, if it is not so, then we can blow up $S$ to obtain $f^{\prime}: X^{\prime} \rightarrow S^{\prime}$ such that $Z^{\prime} \cup \Sigma_{r e d}^{\prime}$ is s.n.c. where $Z^{\prime}$ is the total transform of $Z$. Then, assuming that we only blew up points in $Z$, we have $S^{\prime}-Z^{\prime}=S-Z$, and by [8], Theorem 2.21, $\amalg_{S^{\prime}}(A)=\amalg_{S}(A)$. Thus we have not changed our final result.

We end this section with a few results on local cohomology.

Let $X$ be an arbitrary scheme, and let $Z$ be a closed subset of $X, U=X-Z$, $i: Z \rightarrow X, j: U \rightarrow X$ the inclusions. The functor $i^{!}$which takes sheaves on $X$ to sheaves on $Z$ is defined by

$$
i^{!} \mathcal{F}=\operatorname{ker}\left(i^{*} \mathcal{F} \stackrel{\phi}{\longrightarrow} i^{*} j_{*} j^{*} \mathcal{F}\right)
$$

where $\phi$ is the natural functorial map. (See [15], pg. 76) Equivalently, $\left(i_{*} i \dot{\mathcal{F}}\right)(V)=$ $\operatorname{ker}\left(\mathcal{F}(V) \rightarrow \mathcal{F}\left(V \times_{X} U\right)\right) . H_{Z}^{p}(X, \mathcal{F})$ is the $p$ th derived functor of $\mathcal{F} \mapsto \Gamma\left(Z, i^{!} \mathcal{F}\right)=$ $\operatorname{ker}(\mathcal{F}(X) \rightarrow \mathcal{F}(U))$, and $\underline{H}_{Z}^{p}(X, \mathcal{F})=\left(R^{p} i^{!}\right) \mathcal{F}$. One has the long exact sequence

$$
\cdots \rightarrow H_{Z}^{p}(X, \mathcal{F}) \rightarrow H^{p}(X, \mathcal{F}) \rightarrow H^{p}(U, \mathcal{F}) \rightarrow \cdots .
$$

We will need information about the cohomology of the sheaf $\mathbf{G}_{m}$. Recall from [13] the following facts: $H^{1}\left(X, \mathbf{G}_{m}\right)=\operatorname{Pic} X$, and $H^{i}\left(X, \mathbf{G}_{m}\right)$ is torsion for $i \geq 2$ if $X$ is regular. From the Kummer sequence

$$
0 \rightarrow \mu_{n} \rightarrow \mathbf{G}_{m} \rightarrow \mathbf{G}_{m} \rightarrow 0
$$

we see that we have

$$
0 \rightarrow \operatorname{Pic} X / n \operatorname{Pic} X \rightarrow H^{2}\left(X, \mu_{n}\right) \rightarrow{ }_{n} H^{2}\left(X, \mathbf{G}_{m}\right) \rightarrow 0
$$

which, upon taking the direct limit over all $n$ and choosing identifications $\mu_{n} \cong$ $\mathbf{Z} / n \mathbf{Z}$ (assuming we are working over an algebraically closed ground field of characteristic 0 ) we obtain a sequence for $X$ regular

$$
0 \rightarrow \operatorname{Pic} X \otimes \mathbf{Q} / \mathbf{Z} \rightarrow H^{2}(X, \mathbf{Q} / \mathbf{Z}) \rightarrow H^{2}\left(X, \mathbf{G}_{m}\right) \rightarrow 0 .
$$

Similarly we have exact sequences

$$
0 \rightarrow H^{i-1}\left(X, \mathbf{G}_{m}\right) \otimes \mathbf{Q} / \mathbf{Z} \rightarrow H^{i}(X, \mathbf{Q} / \mathbf{Z}) \rightarrow H^{i}\left(X, \mathbf{G}_{m}\right) \rightarrow 0
$$


for $i \geq 3$, and since $H^{i-1}\left(X, \mathbf{G}_{m}\right)$ is torsion, this yields an isomorphism

$$
H^{i}(X, \mathbf{Q} / \mathbf{Z}) \stackrel{\cong}{\longrightarrow} H^{i}\left(X, \mathbf{G}_{m}\right), \quad i \geq 3 .
$$

Proposition 1.3 (Purity of the Brauer group). Let $X$ be a regular excellent scheme over a field of characteristic $0, Z$ a closed regular subscheme of pure codimension $d$. If $d \geq 2$, then

$$
\underline{H}_{Z}^{i}\left(X, \mathbf{G}_{m}\right)= \begin{cases}0 & \text { for } i \neq 2 d \\ (\mathbf{Q} / \mathbf{Z})_{Z} & \text { for } i=2 d .\end{cases}
$$

If $d=1$, then

$$
\underline{H}_{Z}^{i}\left(X, \mathbf{G}_{m}\right)=0, \quad i=0, i \geq 2,
$$

and

$$
\underline{H}_{Z}^{1}\left(X, \mathbf{G}_{m}\right)=\bigoplus_{i} \mathbf{Z}_{Z_{i}}
$$

where the $Z_{i}$ are the codimension one components of $Z$.

Proof. [13] , p. 134. •

We will need to calculate, given a closed set $Z \subseteq S$, the group $H_{Z}^{2}\left(S, P_{X / S}\right)$ and the sheaf $\underline{H}_{Z}^{2}\left(S, P_{X / S}\right)\left(P_{X / S}=R^{1} f_{*} \mathbf{G}_{m}\right.$ for a map $\left.f: X \rightarrow S\right)$. We will show how to calculate these under the assumption that $Z$ and $f^{-1}(Z)$ are s.n.c. divisors.

Proposition 1.4. Given a map $f: X \rightarrow S$ satisfying the Hypothesis, and $Z \subseteq S$ any closed subset, then

$$
H_{Z}^{2}\left(S, P_{X / S}\right) \cong \frac{H_{f^{-1}(Z)}^{3}\left(X, \mathbf{G}_{m}\right)}{H_{Z}^{3}\left(S, \mathbf{G}_{m}\right)}
$$

Proof. There is a spectral sequence

$$
H_{Z}^{p}\left(S, R^{q} f_{*} \mathcal{F}\right) \Rightarrow H_{f^{-1}(Z)}^{p+q}(X, \mathcal{F})
$$

because

$$
H_{Z}^{0}\left(S, f_{*} \mathcal{F}\right)=H_{f^{-1}(Z)}^{0}(X, \mathcal{F}) .
$$

Since $R^{q} f_{*} \mathbf{G}_{m}=0 \forall q>1$ by $[8,1.3]$, this spectral sequence yields the long exact sequence

$$
\begin{aligned}
\cdots & \rightarrow H_{Z}^{3}\left(S, \mathbf{G}_{m}\right) \rightarrow H_{f^{-1}(Z)}^{3}\left(X, \mathbf{G}_{m}\right) \rightarrow H_{Z}^{2}\left(S, P_{X / S}\right) \\
& \rightarrow H_{Z}^{4}\left(S, \mathbf{G}_{m}\right) \rightarrow H_{f^{-1}(Z)}^{4}\left(X, \mathbf{G}_{m}\right) \rightarrow \cdots
\end{aligned}
$$

Now, as $f: X \rightarrow S$ has a section $\sigma: S \rightarrow X$, one has a composition of natural maps

$$
H_{Z}^{i}\left(S, \mathbf{G}_{m}\right) \stackrel{f^{*}}{\longrightarrow} H_{f^{-1}(Z)}^{i}\left(X, \mathbf{G}_{m}\right) \stackrel{\sigma^{*}}{\longrightarrow} H_{Z}^{i}\left(S, \mathbf{G}_{m}\right)
$$

which must be an isomorphism; thus $f^{*}$ is injective for all $i$ and one obtains the desired result. 
Proposition 1.5. Let $X$ be a regular excellent scheme over an algebraically closed field of characteristic $0, Z$ a s.n.c. divisor with components $Z_{1}, \ldots, Z_{n}$. Then there is an exact sequence

$$
\begin{aligned}
0 \rightarrow \bigoplus_{i} H^{1}\left(Z_{i}, \mathbf{Q} / \mathbf{Z}\right) \rightarrow H_{Z}^{3}\left(X, \mathbf{G}_{m}\right) \rightarrow & \bigoplus_{i<j} H^{0}\left(Z_{i} \cap Z_{j}, \mathbf{Q} / \mathbf{Z}\right) \\
& \stackrel{d}{\longrightarrow} \bigoplus_{k} H^{2}\left(Z_{k}, \mathbf{Q} / \mathbf{Z}\right) \rightarrow H_{Z}^{4}\left(X, \mathbf{G}_{m}\right)
\end{aligned}
$$

where the map $d$ is defined as follows: let $d_{i j k}: H^{0}\left(Z_{i} \cap Z_{j}, \mathbf{Q} / \mathbf{Z}\right) \rightarrow H^{2}\left(Z_{k}, \mathbf{Q} / \mathbf{Z}\right)$ be the natural Gysin map, with $k=i$ or $j$. Then $(d)_{i j}=d_{i j i}-d_{i j j}$.

Proof. If $i: Z \rightarrow X$ is a closed immersion, define $\mathbf{Z}_{Z}=i_{*} i^{*} \mathbf{Z}$. If $Z \subseteq Z^{\prime} \subseteq X$, then there is a natural restriction map $\rho_{Z^{\prime}, Z}: \mathbf{Z}_{Z^{\prime}} \rightarrow \mathbf{Z}_{Z}$. Now, if $Z$ is s.n.c, there is a natural resolution

$$
0 \rightarrow \mathbf{Z}_{Z} \rightarrow A_{0} \rightarrow A_{-1} \rightarrow A_{-2} \rightarrow \cdots
$$

where

$$
A_{-n}=\bigoplus_{i_{0}<\cdots<i_{n}} \mathbf{z}_{Z_{i_{0} \ldots i_{n}}}
$$

with $Z_{i_{0} \ldots i_{n}}=Z_{i_{0}} \cap \cdots \cap Z_{i_{n}}$, and the map $d_{-n}: A_{-n} \rightarrow A_{-(n+1)}$ is defined by

$$
\left(d_{-n} s\right)_{i_{0} \ldots i_{n+1}}=\sum_{j=0}^{n+1}(-1)^{j} \rho_{Z_{i_{0} \ldots \hat{i}_{j} \ldots i_{n+1}}, Z_{i_{0} \ldots i_{n+1}}}\left(s_{i_{0} \ldots \hat{i}_{j} \ldots i_{n+1}}\right) .
$$

Exactness of (1.5) can be checked on stalks. One has $H_{0}(A)=.\mathbf{Z}_{Z}, H_{i}(A)=0,. i \neq$ 0 . Now if $\mathcal{F}$ is a sheaf on $X$, then

$$
\operatorname{Ext}^{i}\left(\mathbf{Z}_{Z}, \mathcal{F}\right)=H_{Z}^{i}(X, \mathcal{F})
$$

Let

$$
0 \rightarrow \mathcal{F} \rightarrow I^{\cdot}
$$

be an injective resolution for $\mathcal{F}$, and consider the bicomplex $M^{*}=\operatorname{Hom}\left(A ., I^{*}\right)$, with differentiation maps $d_{I}: \operatorname{Hom}\left(A_{p}, I^{q}\right) \rightarrow \operatorname{Hom}\left(A_{p+1}, I^{q}\right)$ and $d_{I I}: \operatorname{Hom}\left(A_{p}, I^{q}\right)$ $\rightarrow \operatorname{Hom}\left(A_{p}, I^{q+1}\right)$. One thus obtains two spectral sequences

$$
\begin{aligned}
{ }_{I} E_{1}^{p, q} & =\operatorname{Ext}^{q}\left(A_{p}, \mathcal{F}\right) \Rightarrow H^{p+q}\left(\operatorname{Tot}\left(M^{* *}\right)\right) \\
{ }_{I I} E_{1}^{p, q} & =\operatorname{Hom}\left(H_{q}(A .), I^{p}\right) \Rightarrow H^{p+q}\left(\operatorname{Tot}\left(M^{*}\right)\right)
\end{aligned}
$$

From the second, we deduce that $H^{p}\left(\operatorname{Tot}\left(M^{\cdot \cdot}\right)\right)=\operatorname{Ext}^{p}\left(\mathbf{Z}_{Z}, \mathcal{F}\right)=H_{Z}^{p}(X, \mathcal{F})$, so we obtain from the first a second quadrant spectral sequence

$$
E_{1}^{p, q}=\bigoplus_{i_{0}<\cdots<i_{-p}} H_{Z_{i_{0} \cdots i_{-p}}}^{q}(X, \mathcal{F}) \Rightarrow H_{Z}^{p+q}(X, \mathcal{F}) .
$$

Calculating the $E_{1}^{p, q}$ terms in our situation, using Proposition 1.3 and the spectral sequence

$$
E_{2}^{p, q}=H^{p}\left(Z, \underline{H}_{Z}^{q}\left(X, \mathbf{G}_{m}\right)\right) \Rightarrow H_{Z}^{p+q}\left(X, \mathbf{G}_{m}\right),
$$

one can calculate all the relevant terms to obtain the desired exact sequence, and, keeping in mind that $d: \bigoplus_{i<j} H_{Z_{i j}}^{4}\left(X, \mathbf{G}_{m}\right) \rightarrow \bigoplus_{k} H_{Z_{k}}^{4}\left(X, \mathbf{G}_{m}\right)$ is induced by the map $d_{0}: A_{0} \rightarrow A_{-1}$, we obtain the description of the map $d$. • 
Proposition 1.6. Let $f: X \rightarrow S$ satisfy the Hypothesis. If $Z \subseteq S$ is a s.n.c. divisor with $f^{-1}(Z)$ a s.n.c. divisor in $X$, with $Z=Z_{1} \cup \ldots \cup Z_{m}, f^{-1}\left(Z_{i}\right)=$ $Y_{i}^{1} \cup \ldots \cup Y_{i}^{n_{i}}$, and $k: f^{-1}(Z) \rightarrow Z$ the restriction of $f$, then we have a sheaf $\mathcal{F}$ defined by

$$
0 \longrightarrow \bigoplus_{i<j}(\mathbf{Q} / \mathbf{Z})_{Z_{i} \cap Z_{j}} \stackrel{\alpha}{\longrightarrow} \bigoplus_{(i, j)<\left(i^{\prime}, j^{\prime}\right)} k_{*}(\mathbf{Q} / \mathbf{Z})_{Y_{i}^{j} \cap Y_{i^{\prime}}^{j^{\prime}}} \longrightarrow \mathcal{F} \longrightarrow 0
$$

giving

$$
0 \longrightarrow \bigoplus_{i, j} R^{1} k_{*}(\mathbf{Q} / \mathbf{Z})_{Y_{i}^{j}} \longrightarrow \underline{H}_{Z}^{2}\left(S, P_{X / S}\right) \longrightarrow \mathcal{F} \stackrel{d}{\longrightarrow} \bigoplus_{i, j} R^{2} k_{*}(\mathbf{Q} / \mathbf{Z})_{Y_{i}^{j}}
$$

where $(i, j)<\left(i^{\prime}, j^{\prime}\right)$ if $i<i^{\prime}$ or $i=i^{\prime}$ and $j<j^{\prime}$, and the map $\alpha$, over a point $P$ of $Z_{i} \cap Z_{j}$, can be identified with

$$
\mathbf{Q} / \mathbf{Z} \rightarrow(\mathbf{Q} / \mathbf{Z})^{c}
$$

where $c$ is the number of irreducible components of the fibre over $P$, given by $a \mapsto$ $\left(m_{i} a\right)$ with $m_{i}$ being the multiplicity of the ith component of the fibre. The mapd is the relative version of the map d given in Proposition 1.5.

Proof. We have the diagram

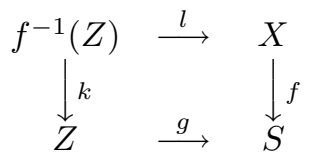

Note that $k_{*} l^{!}=g^{!} f_{*}$. Indeed, if $\mathcal{F}$ is a sheaf on $X, U$ étale over $S$, then

$$
\begin{aligned}
\left(g_{*} k_{*} l^{!} \mathcal{F}\right)(U) & =\left(f_{*} l_{*} l^{!} \mathcal{F}\right)(U) \\
& =\left(l_{*} l \cdot \mathcal{F}\right)\left(U \times_{S} X\right) \\
& =\operatorname{ker}\left(\mathcal{F}\left(U \times_{S} X\right) \rightarrow \mathcal{F}\left(U \times_{S} X-U \times_{S} f^{-1}(Z)\right)\right) \\
& =\operatorname{ker}\left(f_{*} \mathcal{F}(U) \rightarrow f_{*} \mathcal{F}\left(U-U \times_{S} Z\right)\right) \\
& =\left(g_{*} g^{!} f_{*} \mathcal{F}\right)(U) .
\end{aligned}
$$

Since $g_{*}$ is fully faithful ([15, II $\left.\left.3.14 \mathrm{~d}\right)\right]$ ), the claim follows.

We have the spectral sequence

$$
\left(R^{p} g^{!}\right)\left(R^{q} f_{*}\right) \mathbf{G}_{m} \Rightarrow R^{p+q}\left(g^{!} f_{*}\right) \mathbf{G}_{m}=R^{p+q}\left(k_{*} l^{!}\right) \mathbf{G}_{m},
$$

and since $f_{*} \mathbf{G}_{m}=\mathbf{G}_{m}$ and $R^{q} f_{*} \mathbf{G}_{m}=0 \forall q>1$ ([8], 1.4), the spectral sequence degenerates, giving, in part, the exact sequence

$$
\underline{H}_{Z}^{3}\left(S, \mathbf{G}_{m}\right) \rightarrow R^{3}\left(g^{!} f_{*}\right) \mathbf{G}_{m} \rightarrow \underline{H}_{Z}^{2}\left(S, R^{1} f_{*} \mathbf{G}_{m}\right) \rightarrow \underline{H}_{Z}^{4}\left(S, \mathbf{G}_{m}\right) .
$$

Now by the exact sequence (1.5) for $Z$ and the fact that

$$
\underline{\operatorname{Ext}}^{i}\left(\mathbf{Z}_{Z}, \mathcal{F}\right)=g_{*} \underline{\operatorname{Ext}}^{i}\left(\mathbf{Z}, g^{!} \mathcal{F}\right)=g_{*} \underline{H}_{Z}^{i}(S, \mathcal{F})
$$

([15] , pg. 242) we see that we have, by Proposition 1.3,

$$
\underline{H}_{Z}^{3}\left(S, \mathbf{G}_{m}\right)=\bigoplus_{i<j} \underline{H}_{Z_{i} \cap Z_{j}}^{4}\left(S, \mathbf{G}_{m}\right)=\bigoplus_{i<j}(\mathbf{Q} / \mathbf{Z})_{Z_{i} \cap Z_{j}} \quad \text { and } \quad \underline{H}_{Z}^{4}\left(S, \mathbf{G}_{m}\right)=0 .
$$

Now

$$
g_{*} R^{q}\left(g^{!} f_{*}\right) \mathbf{G}_{m}=R^{q}\left(g_{*} k_{*} l^{!}\right) \mathbf{G}_{m}=R^{q}\left(f_{*} l_{*} l^{!}\right) \mathbf{G}_{m}=R^{q}\left(f_{*} \underline{\operatorname{Hom}}\left(\mathbf{Z}_{f-1}(Z), \cdot\right)\right) \mathbf{G}_{m} .
$$


If $0 \rightarrow \mathbf{G}_{m} \rightarrow I^{\cdot}$ is an injective resolution of $\mathbf{G}_{m}$, then as in the proof of Proposition 1.5 we obtain the bicomplex $M^{*}=f_{*} \underline{\operatorname{Hom}}\left(A ., I^{*}\right)$ and thus obtain a spectral sequence

$$
\begin{aligned}
E_{1}^{p, q}=\bigoplus_{i_{0}<\ldots<i_{-p}} R^{q}\left(f_{*} \underline{\operatorname{Hom}}\left(\mathbf{Z}_{Y_{i_{0} \ldots i-p}}, \cdot\right)\right) \mathbf{G}_{m} & =\bigoplus_{i_{0}<\ldots<i_{-p}} g_{*} R^{q}\left(k_{*} l_{i_{0} \ldots i_{-p}}^{!}\right) \mathbf{G}_{m} \\
& \Rightarrow g_{*} R^{p+q}\left(k_{*} l^{!}\right) \mathbf{G}_{m}
\end{aligned}
$$

where $l_{i_{0} \ldots i_{n}}: Y_{i_{0} \ldots i_{n}} \rightarrow X$ is the inclusion, $Y_{i_{0} \ldots i_{n}}$ denoting the intersection of $n+1$ components of $f^{-1}(Z)$. Now one proceeds as before, using the spectral sequence

$$
\left(R^{p} k_{*}\right)\left(R^{q} l_{i_{0} \ldots i_{n}}^{!}\right) \mathbf{G}_{m} \Rightarrow R^{p+q}\left(k_{*} l_{i_{0} \ldots i_{n}}^{!}\right) \mathbf{G}_{m}
$$

to compute the various terms. We see that

$$
R^{q}\left(k_{*} l_{i_{0}}^{!}\right) \mathbf{G}_{m}= \begin{cases}0, & q=0, \\ \left(R^{q-1} k_{*}\right)\left(l_{i_{0}}^{*} \mathbf{Z}\right), & q \geq 1,\end{cases}
$$

with $\left(R^{q-1} k_{*}\right)\left(l_{i_{0}}^{*} \mathbf{Z}\right)=\left(R^{q-2} k_{*}\right)\left(l_{i_{0}}^{*} \mathbf{Q} / \mathbf{Z}\right)$ for $q \geq 3$, and

$$
R^{q}\left(k_{*} l_{i_{0} i_{1}}^{!}\right) \mathbf{G}_{m}= \begin{cases}0, & q \leq 3, \\ \left(R^{q-4} k_{*}\right)\left(l_{i_{0} i_{1}}^{*} \mathbf{Q} / \mathbf{Z}\right), & q \geq 4 .\end{cases}
$$

Dropping the $g_{*}$ 's by [15], II Cor 3.14 d), one thus obtains an exact sequence

$$
\begin{aligned}
& 0 \rightarrow \bigoplus_{i, j} R^{1} k_{*}(\mathbf{Q} / \mathbf{Z})_{Y_{i}^{j}} \rightarrow R^{3}\left(g^{!} f_{*}\right) \mathbf{G}_{m} \rightarrow \bigoplus_{(i, j)<\left(i^{\prime}, j^{\prime}\right)} k_{*}(\mathbf{Q} / \mathbf{Z})_{Y_{i}^{j} \cap Y_{i^{\prime}}^{j^{\prime}}} \\
& \rightarrow \bigoplus_{i, j} R^{2} k_{*}(\mathbf{Q} / \mathbf{Z})_{Y_{i}^{j}} .
\end{aligned}
$$

Take $\alpha$ to be the composed map

$$
\bigoplus_{i<j}(\mathbf{Q} / \mathbf{Z})_{Z_{i} \cap Z_{j}}=\underline{H}_{Z}^{3}\left(S, \mathbf{G}_{m}\right) \rightarrow R^{3}\left(g^{!} f_{*}\right) \mathbf{G}_{m} \rightarrow \bigoplus_{(i, j)<\left(i^{\prime}, j^{\prime}\right)} k_{*}(\mathbf{Q} / \mathbf{Z})_{Y_{i}^{j} \cap Y_{i^{\prime}}^{j^{\prime}}}
$$

where the first arrow is from (1.6). This map $\alpha$ takes the desired form, and is then injective since $f: X \rightarrow S$ has a section. This yields a diagram of exact sequences

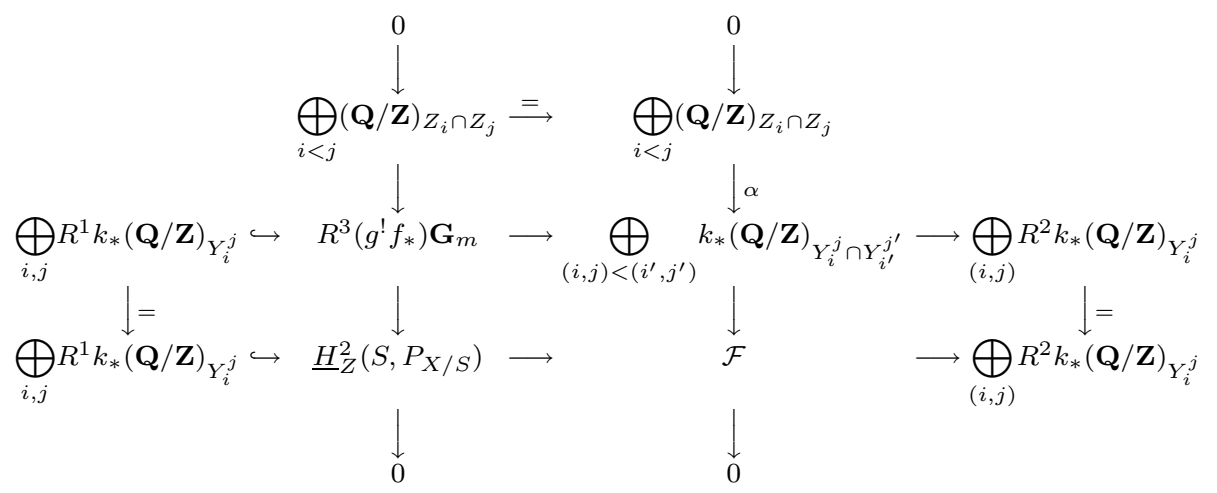

This yields the desired sequences. 


\section{The calculation of $H_{Z}^{2}\left(S, i_{*} A\right)$}

We continue to work in the setting of the Additional Hypothesis of $\S 1$.

Lemma 2.1. Let $i^{\prime}: \eta \rightarrow S-Z$ be the inclusion of the generic point in $S-Z$. Then

$$
\begin{gathered}
\left.\left(i_{*} A\right)\right|_{S-Z}=i_{*}^{\prime} A, \\
\left.P_{X / S}\right|_{S-Z}=P_{\left(X-f^{-1}(Z) / S-Z\right)}, \\
\left.\left(i_{*} i^{*} P_{X / S}\right)\right|_{S-Z}=i_{*}^{\prime} i^{\prime *}\left(\left.P_{X / S}\right|_{S-Z}\right) .
\end{gathered}
$$

In particular, $\amalg_{S-Z}(A)=H^{1}\left(S-Z, i_{*} A\right)$ and we have an exact sequence

$$
0 \rightarrow \amalg_{S}(A) \rightarrow \amalg_{S-Z}(A) \rightarrow H_{Z}^{2}\left(S, i_{*} A\right) \rightarrow H^{2}\left(S, i_{*} A\right) .
$$

Proof. Obvious.

\section{Proposition 2.2.}

a) $\underline{H}_{Z}^{0}\left(S, i_{*} A\right)=\underline{H}_{Z}^{1}\left(S, i_{*} A\right)=0$, and $H_{Z}^{2}\left(S, i_{*} A\right)=H^{0}\left(\underline{H}_{Z}^{2}\left(S, i_{*} A\right)\right)$.

b) $\underline{H}_{Z}^{i}\left(S, i_{*} A\right)=\underline{H}_{Z}^{i}\left(S, i_{*} i^{*} P_{X / S}\right)$ for $i \leq 2$, and in addition for $i=3$ if $Z$ is pure codimension 2 .

c) There is an exact sequence

$$
\begin{aligned}
0 & \rightarrow \underline{H}_{Z}^{2}(S, \mathcal{E}) \rightarrow \underline{H}_{Z}^{2}\left(S, P_{X / S}\right) \rightarrow \underline{H}_{Z}^{2}\left(S, i_{*} A\right) \\
& \rightarrow \underline{H}_{Z}^{3}(S, \mathcal{E}) \rightarrow \underline{H}_{Z}^{3}\left(S, P_{X / S}\right) \rightarrow \underline{H}_{Z}^{3}\left(S, i_{*} i^{*} P_{X / S}\right)
\end{aligned}
$$

Proof. a) If $j: Z \rightarrow S$ is the inclusion, then $j_{*} \underline{H}_{Z}^{i}\left(S, i_{*} A\right)$ is the sheaf associated with the presheaf $U \mapsto H_{U \times_{S} Z}^{i}\left(U,\left.\left(i_{*} A\right)\right|_{U}\right)$. Thus it is sufficient to show that $H_{Z}^{i}\left(S, i_{*} A\right)=0, i=0,1$ for any $Z$ and $S, S$ irreducible. Indeed, $H^{0}\left(S, i_{*} A\right)=$ $H^{0}\left(S-Z, i_{*} A\right)=A(K)$, and $\amalg_{S}(A) \rightarrow \amalg_{S-Z}(A)$ is necessarily an injection. This proves the claim. The last statement then follows from the spectral sequence

$$
H^{p}\left(Z, \underline{H}_{Z}^{q}\left(S, i_{*} A\right)\right) \Rightarrow H_{Z}^{p+q}\left(S, i_{*} A\right) .
$$

b) This follows from the exact sequence (1.2). Taking the long exact sequence obtained by applying the functor $j^{!}$, and using the fact that $\underline{H}_{Z}^{i}(S, \mathbf{Z})=\underline{H}_{Z}^{i-1}(S, \mathbf{Q} / \mathbf{Z})$ $=0$ for $i-1<2 \operatorname{codim}(Z)$ by purity, we obtain the desired isomorphisms.

c) This follows by applying $j$ ! to (1.1) and using a) and b).

We will first calculate $\underline{H}_{Z}^{2}\left(S, i_{*} A\right)$ and $\underline{H}_{Z}^{3}\left(S, i_{*} A\right)$ when $Z$ is codimension 2 . In this case, these sheaves are supported on a finite set of closed points and thus are entirely determined by their stalks at these points. These stalks have already been calculated in $[8]$.

Theorem 2.3. Suppose $\bar{f}: \bar{X} \rightarrow \bar{S}$ is a flat proper morphism of regular schemes with a section with $\bar{S}$ strictly local, of dimension 2 , with closed point $s$, and generic fibre $A$ an elliptic curve. Then there is an exact sequence, applying Theorem 1.1 to $f: X \rightarrow S$ where $S=\bar{S}-\{s\}$ and $X=\bar{X} \times_{\bar{S}} S$,

$$
0 \rightarrow \amalg_{S}(A) \rightarrow H^{2}(S, \mathcal{E}) \rightarrow H^{2}\left(S, P_{X / S}\right) \rightarrow H^{2}\left(S, i_{*} A\right) \rightarrow 0 .
$$


The groups $H^{2}(S, \mathcal{E})$ and $H^{2}\left(S, P_{X / S}\right)$ and the map between them are defined by the commutative diagram

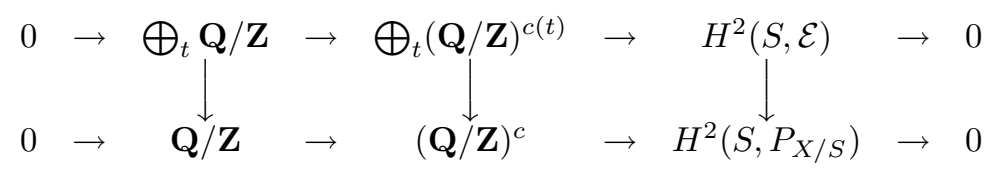

where the sum is over all $t \in S^{(1)}$ such that $X_{t}$ is not geometrically irreducible, $c(t)$ is the number of components of $X_{t}$, and $c$ is the number of components of the fibre $X_{0}$ over the closed point of $\bar{S}$. The first arrow in the first row is given for a given $t$ by

$$
a \mapsto\left(m_{i} r_{i} a\right)
$$

where $m_{i}$ is the multiplicity of $X_{t}^{i}$ and $r_{i}$ is the ramification index of $C\left(t_{i}\right) \rightarrow C(t)$ at $s$. The first arrow in the second row is given by $a \mapsto\left(m_{i} a\right)$ where $m_{i}$ is the multiplicity of the ith component of $X_{0}$. The first vertical arrow is summation and the second takes $X_{t}^{i}$ to the sum of components of the central fibre of $\bar{X}_{t}^{i} \rightarrow$ $C\left(t_{i}\right)$ taken with the appropriate multiplicities, where $\bar{X}_{t}^{i}$ is the normalization of the closure of $X_{t}^{i}$ in $\bar{X}$.

Proof. See [8] , Theorem 3.1. (One can reconstruct the proof efficiently by using Propositions 1.3, 1.4 and 3.1 of this paper.)

Theorem 2.4. Given the hypothesis of Theorem 2.3, assume furthermore that $\Sigma$ consists of two components meeting transversally, of fibre type $\alpha$ and $\beta$ respectively. Then the values of $H^{1}\left(S, i_{*} A\right)=H_{s}^{2}\left(\bar{S}, i_{*} A\right)$ and $H^{2}\left(S, i_{*} A\right)=H_{s}^{3}\left(\bar{S}, i_{*} A\right)$ are given by the following table, depending on $\alpha$ and $\beta$. This covers all possible collisions occurring in a Miranda model.

$\begin{array}{lll}\alpha, \beta & H^{1}\left(S, i_{*} A\right) & H^{2}\left(S, i_{*} A\right) \\ I_{M_{1}}, I_{M_{2}}, M_{1}, M_{2} \geq 1 & 0 & \mathbf{Q} / \mathbf{Z} \\ \left.\text { (not both } M_{1} \text { and } M_{2} \text { odd }\right) & & \\ I_{M_{1}}, I_{M_{2}}^{*}, M_{1} \text { odd } & 0 & 0 \\ I_{M_{1}}, I_{M_{2}}^{*}, M_{1}>0, \text { even } & \mathbf{Z} / 2 \mathbf{Z} & 0 \\ I I, I V & 0 & 0 \\ I I, I_{0}^{*} & 0 & 0 \\ I I, I V^{*} & 0 & 0 \\ I V, I_{0}^{*} & 0 & 0 \\ I I I, I_{0}^{*} & \mathbf{Z} / 2 \mathbf{Z} & 0\end{array}$

Proof. One calculates using Theorem 2.3 and our modification of the explicit resolution given by Miranda. In the cases $I_{M_{1}}+I_{M_{2}}$ and $I_{M_{1}}+I_{M_{2}}^{*}$ we give some details of the resolution, because they will be vital cases later. In Table 1, we give some details for $I_{M_{1}}+I_{M_{2}}$. The central fibre consists of a cycle of $\mathbf{P}^{1}$ 's, $f_{1}, \ldots, f_{M_{1}^{\prime}+M_{2}}$, $M_{1}^{\prime}=M_{1}$ if $M_{1}$ is even and $M_{1}+1$ if $M_{1}$ is odd. Each $Y_{i}$ or $Y_{j}^{\prime}$ specialises to a sum of these curves, with no multiplicities. This is given by the $f_{i}$ 's listed in the second column of the table. The rest of the information in the table can be ignored for now. So, for example, we work out the calculation for the collision $I_{2}+I_{2}$. We 
can give the matrix of the $\operatorname{map}(\mathbf{Q} / \mathbf{Z})^{4} \rightarrow(\mathbf{Q} / \mathbf{Z})^{4}$ by

$$
\begin{aligned}
& Y_{1} Y_{2} Y_{1}^{\prime} Y_{2}^{\prime} \\
& \begin{array}{l}
f_{1} \\
f_{2} \\
f_{3} \\
f_{4}
\end{array}\left(\begin{array}{llll}
1 & 0 & 0 & 1 \\
1 & 0 & 0 & 1 \\
1 & 0 & 1 & 0
\end{array}\right)
\end{aligned}
$$

In other words, $Y_{1}$ specialises to $f_{1}+f_{3}+f_{4}, Y_{2}$ specialises to $f_{2}$, etc. From this one obtains the desired results. The information for $I_{M_{1}}+I_{M_{2}}^{*}$ is presented in Table 2 in a similar fashion, except that now some of the central fibres occur with multiplicities in the specialisation. These are represented by coefficients in the entries in column 2. One such example has already been worked out in [8] .

We also calculate here the $I_{0}^{*}+I I I$ example. In Miranda's resolution, if $\Sigma=$ $\Sigma_{1} \cup \Sigma_{2}$ with $\Sigma_{1}$ of fibre type $I_{0}^{*}$ and $\Sigma_{2}$ of fibre type $I I I$, one has $f^{-1}\left(\Sigma_{1}\right)=$ $Y_{1}+Y_{2}+2 Y_{3}+Y_{4}$ where $Y_{1}, Y_{3}$ and $Y_{4}$ are ruled over $\Sigma_{1}$ and $Y_{2}$ is ruled over a double cover of $\Sigma_{1}$ branched at the closed point of $\bar{S}$. $f^{-1}\left(\Sigma_{2}\right)=Y_{1}^{\prime}+Y_{2}^{\prime}$, both ruled surfaces over $\Sigma_{2}$. The central fibre is

$$
\bar{X}_{m}=f_{1}+2 f_{2}+3 f_{3}+2 f_{4}+f_{5}
$$

with specialization matrix

$$
\begin{aligned}
& \begin{array}{llllll}
Y_{1} & Y_{2} & Y_{3} & Y_{4} & Y_{1}^{\prime} & Y_{2}^{\prime}
\end{array} \\
& f_{1}\left(\begin{array}{llllll}
1 & 0 & 0 & 0 & 1 & 0
\end{array}\right) \\
& f_{2}\left(\begin{array}{llllll}
2 & 0 & 0 & 0 & 0 & 2
\end{array}\right. \\
& \begin{array}{lllllll}
f_{3} & 1 & 1 & 0 & 0 & 0 & 3
\end{array} \\
& f_{4}\left(\begin{array}{llllll}
0 & 0 & 1 & 0 & 0 & 2 \\
0 & 0 & 0 & 1 & 0 & 1
\end{array}\right.
\end{aligned}
$$

from which we see that we obtain

$$
\left(\begin{array}{llllll}
0 & 1 / 2 & 0 & 1 / 2 & 0 & 1 / 2
\end{array}\right)
$$

as a representative of the non-zero element of $H_{s}^{2}\left(\bar{S}, i_{*} A\right) \subseteq H_{s}^{3}(\bar{S}, \mathcal{E})$.

Definition. A point $P \in S$ is obstructed if $\underline{H}_{P}^{3}\left(S, i_{*} A\right) \neq 0$.

Corollary 2.5. If $X \rightarrow S$ is a modified Miranda model, then the obstructed points are precisely the points which are at collisions of type $I_{M_{1}}+I_{M_{2}}$.

Proof. By Theorem 2.3, it is easy to see that if $P$ is not a collision point, then $\underline{H}_{P}^{2}\left(S, i_{*} A\right)=\underline{H}_{P}^{3}\left(S, i_{*} A\right)=0$. Otherwise, we refer to Theorem 2.4.

We now proceed to the case that $\operatorname{dim} Z=1$. The very simplest case proves to be virtually enough to complete the calculations, in conjunction with the above calculations.

Proposition 2.6. Let $Z \subseteq S$ be a smooth curve with $Z \cap \Sigma=\varnothing$. Then

$$
\underline{H}_{Z}^{2}\left(S, i_{*} A\right)=R^{1} k_{*} \mathbf{Q} / \mathbf{Z}
$$

where $k: f^{-1}(Z) \rightarrow Z$ is the restriction of $f: X \rightarrow S$.

Proof. Because the support of $\mathcal{E}$ is contained in $\Sigma$, the condition that $Z \cap \Sigma=$ $\varnothing$ implies that $\underline{H}_{Z}^{i}(S, \mathcal{E})=0 \forall i$. Thus, by Proposition $\left.2.2 \mathrm{c}\right), \underline{H}_{Z}^{2}\left(S, i_{*} A\right) \cong$ $\underline{H}_{Z}^{2}\left(S, P_{X / S}\right)$. The claim then follows immediately from Proposition 1.6. 
Recall that the Mordell-Weil group of a jacobian elliptic surface (i.e., an elliptic surface having a section) is the group of sections, or alternatively, the group of points of the generic fibre rational over the function field of the base. We shall always take the given section $\sigma_{0}: S \rightarrow X$ of the Hypothesis as the zero section.

Proposition 2.7. Let $k: Y \rightarrow C$ be a jacobian elliptic fibration with no reducible fibres, $\operatorname{dim} C=1$. Then

$$
H^{0}\left(C, R^{1} k_{*} \mathbf{Q} / \mathbf{Z}\right)=M W(Y / C)_{t o r s}
$$

the torsion part of the Mordell-Weil group of $Y$ over $C$.

Proof. $R^{1} k_{*} \mathbf{G}_{m}$ is the relative Picard sheaf, the sheaf associated to the presheaf

$$
U \mapsto \operatorname{Pic}\left(U \times_{C} Y\right) / \operatorname{Pic}(U) .
$$

Since $k: Y \rightarrow C$ has a section, this is in fact already a sheaf. By the Kummer sequence, we have

$$
0 \rightarrow R^{1} k_{*} \mu_{n} \rightarrow R^{1} k_{*} \mathbf{G}_{m} \rightarrow R^{1} k_{*} \mathbf{G}_{m},
$$

where the last arrow is given by multiplication by $n$. Thus $R^{1} k_{*} \mu_{n}$ is the sheaf given by

$$
U \mapsto{ }_{n}\left(\operatorname{Pic}\left(U \times{ }_{C} Y\right) / \operatorname{Pic} U\right)={ }_{n} M W\left(U \times{ }_{C} Y / U\right),
$$

the last equality since all fibres are irreducible. Finally, we have

$$
R^{1} k_{*} \mathbf{Q} / \mathbf{Z}=\lim _{\longrightarrow} R^{1} k_{*} \mu_{n}
$$

Proposition 2.8. Suppose that $Z \subseteq S$ consists of a union of non-singular curves $Z_{1}, \ldots, Z_{n}$ such that no $Z_{i}$ is contained in $\Sigma$ and $Z$ contains no obstructed points or points $P$ with $H_{P}^{2}\left(S, i_{*} A\right) \neq 0$. Then

$$
H_{Z}^{2}\left(S, i_{*} A\right)=\bigoplus_{i=1}^{n} M W\left(f^{-1}\left(Z_{i}\right) / Z_{i}\right)_{\text {tors }} .
$$

Proof. Let $Z^{\prime}=(\operatorname{sing} Z) \cup(Z \cap \Sigma)$. By the hypothesis, $H_{Z^{\prime}}^{2}\left(S, i_{*} A\right)=H_{Z^{\prime}}^{3}\left(S, i_{*} A\right)=$ 0 , and thus, by [15, III, 1.26], we have an exact sequence

$$
0=H_{Z^{\prime}}^{2}\left(S, i_{*} A\right) \rightarrow H_{Z}^{2}\left(S, i_{*} A\right) \rightarrow H_{Z-Z^{\prime}}^{2}\left(S-Z^{\prime}, i_{*} A\right) \rightarrow H_{Z^{\prime}}^{3}\left(S, i_{*} A\right)=0,
$$

so

$$
H_{Z}^{2}\left(S, i_{*} A\right) \cong H_{Z-Z^{\prime}}^{2}\left(S-Z^{\prime}, i_{*} A\right) .
$$

The latter is calculated by Propositions 2.6 and 2.7 :

$$
\begin{aligned}
H_{Z-Z^{\prime}}^{2}\left(S-Z^{\prime}, i_{*} A\right) & =\bigoplus_{i=1}^{n} H^{0}\left(Z_{i}-Z^{\prime}, R^{1} k_{i *} \mathbf{Q} / \mathbf{Z}\right) \\
& =\bigoplus_{i=1}^{n} M W\left(f^{-1}\left(Z_{i}-Z^{\prime}\right) / Z_{i}-Z^{\prime}\right)_{t o r s} \\
& =\bigoplus_{i=1}^{n} M W\left(f^{-1}\left(Z_{i}\right) / Z_{i}\right)_{\text {tors }}
\end{aligned}
$$

where $k_{i}: f^{-1}\left(Z_{i}-Z^{\prime}\right) \rightarrow Z_{i}-Z^{\prime}$ is the restriction. 
In other words, by removing unobstructed points from $S$, we do not change the calculation at all.

Since the $I_{M_{1}}+I_{M_{2}}$ collisions are obstructed, in order to understand what happens there, we need to do a local calculation at the collision point itself; we cannot hide the collisions as we did above. We note that the following local calculation agrees with that performed in [19] using different methods.

Proposition 2.9. Let $\bar{S}$ be strictly local of dimension $2, f: \bar{X} \rightarrow \bar{S}$ a modified Miranda model, $\Sigma=\Sigma_{1} \cup \Sigma_{2}, \Sigma_{1}$ and $\Sigma_{2}$ the two irreducible components of the discriminant locus, $\Sigma_{1}$ and $\Sigma_{2}$ meeting transversally with fibre type $I_{M_{1}}$ and $I_{M_{2}}$ respectively, $M_{1}, M_{2}>0$, and $M_{2}$ even. Then if $S=\bar{S}-\Sigma_{1} \cup \Sigma_{2}, X=S \times_{\bar{S}} \bar{X}$, we have

$$
\amalg_{S}(A) \subseteq \mathbf{Q} / \mathbf{Z} \oplus \mathbf{Q} / \mathbf{Z}
$$

with

$$
\amalg_{S}(A)=\left\{(b, c) \mid b, c \in \mathbf{Q} / \mathbf{Z} \text { and } M_{2} b=M_{1} c\right\} .
$$

Proof. By Theorem 1.1, $\amalg_{S}(A)=H^{2}\left(X, \mathbf{G}_{m}\right) / H^{2}\left(S, \mathbf{G}_{m}\right)$, and, since $H^{i}\left(\bar{X}, \mathbf{G}_{m}\right)$ $=H^{i}\left(\bar{S}, \mathbf{G}_{m}\right)=0, i \geq 2$, putting $Z=\Sigma_{1} \cup \Sigma_{2}$,

$$
H^{2}\left(X, \mathbf{G}_{m}\right) / H^{2}\left(S, \mathbf{G}_{m}\right)=H_{f^{-1}(Z)}^{3}\left(\bar{X}, \mathbf{G}_{m}\right) / H_{Z}^{3}\left(\bar{S}, \mathbf{G}_{m}\right) .
$$

Put

$$
M_{1}^{\prime}:= \begin{cases}M_{1} & \text { if } M_{1} \text { is even, } \\ M_{1}+1 & \text { if } M_{1} \text { is odd }\end{cases}
$$

Now $f^{-1}(Z)$ is a s.n.c. divisor consisting of divisors $Y_{1}, \ldots, Y_{M_{1}^{\prime}}, Y_{1}^{\prime}, \ldots, Y_{M_{2}}^{\prime}$, following the notation of Table 1. For notational convenience, set

$$
Y_{M_{1}^{\prime}+j}:=Y_{j}^{\prime}, \quad 1 \leq j \leq M_{2} .
$$

Noting that the $Y_{i}$ are ruled surfaces over a strictly local base, we have $H^{1}\left(Y_{i}, \mathbf{Q} / \mathbf{Z}\right)$ $=0, \forall i$, and thus by Prop. 1.5, we have an exact sequence

$$
0 \rightarrow H_{f^{-1}(Z)}^{3}\left(\bar{X}, \mathbf{G}_{m}\right) \rightarrow \bigoplus_{i<j} H^{0}\left(Y_{i} \cap Y_{j}, \mathbf{Q} / \mathbf{Z}\right) \stackrel{d}{\longrightarrow} \bigoplus_{k} H^{2}\left(Y_{k}, \mathbf{Q} / \mathbf{Z}\right) .
$$

Now, by proper base change, $H^{2}\left(Y_{k}, \mathbf{Q} / \mathbf{Z}\right)=H^{2}\left(\left(Y_{k}\right)_{0}, \mathbf{Q} / \mathbf{Z}\right)=\bigoplus_{i=1}^{c_{k}} H^{2}\left(f_{k}^{i}, \mathbf{Q} / \mathbf{Z}\right)$, where $\left(Y_{k}\right)_{0}$ is the central fibre and the $f_{k}^{i}, 1 \leq i \leq c_{k}$, its components. The Gysin map $H^{0}\left(Y_{i} \cap Y_{j}, \mathbf{Q} / \mathbf{Z}\right) \rightarrow H^{2}\left(Y_{i}, \mathbf{Q} / \mathbf{Z}\right)$ then is obtained by $a\left[Y_{i} \cap Y_{j}\right] \rightarrow$ $\left(a\left[Y_{i} \cap Y_{j}\right] . f_{i}^{k}\right)$. These intersection numbers are given in the third column of Table 1. Following the notation there, $f_{1}, \ldots, f_{M_{1}+M_{2}}$ are the components of the central fibre of $\bar{X} \rightarrow \bar{S}$, the $C_{i}$ are the curves of intersection between the components of $f^{-1}\left(\Sigma_{1}\right)$, and the $C_{j}^{\prime}$ are the curves of intersection between the components of $f^{-1}\left(\Sigma_{2}\right)$. Column 2 of Table 1 shows those curves among these listed contained in each component of $f^{-1}(Z)$.

Let

$$
\begin{aligned}
\left(a_{1}, \ldots, a_{M_{1}^{\prime}+M_{2}}, b_{1}, \ldots, b_{M_{1}^{\prime}}, c_{1}, \ldots, c_{M_{2}}\right) \in \bigoplus_{i<j} H^{0}\left(Y_{i} \cap Y_{j}, \mathbf{Q} / \mathbf{Z}\right) \\
=\bigoplus_{i=1}^{M_{1}^{\prime}+M_{2}} H^{0}\left(f_{i}, \mathbf{Q} / \mathbf{Z}\right) \oplus \bigoplus_{i=1}^{M_{1}^{\prime}} H^{0}\left(C_{i}, \mathbf{Q} / \mathbf{Z}\right) \oplus \bigoplus_{i=1}^{M_{2}} H^{0}\left(C_{i}^{\prime}, \mathbf{Q} / \mathbf{Z}\right) .
\end{aligned}
$$


The image under $d$ will be an element of

$$
\bigoplus_{i=1}^{M_{1}^{\prime}+M_{2}} H^{2}\left(Y_{i}, \mathbf{Q} / \mathbf{Z}\right)=\bigoplus_{i=1}^{M_{1}^{\prime}+M_{2}} H^{2}\left(f_{i}, \mathbf{Q} / \mathbf{Z}\right) \oplus \bigoplus_{i=1}^{M_{1}^{\prime}+M_{2}} H^{2}\left(f_{i}, \mathbf{Q} / \mathbf{Z}\right)
$$

We first consider the case $M_{1}$ even. Setting the image of this element under $d$ equal to zero, we obtain $2\left(M_{1}+M_{2}\right)$ equations, using the intersection numbers in the third column of Table 1 . The row labelled $f_{i}$ in the following table gives the values of the image of $\left(a_{1}, \ldots, c_{M_{2}}\right)$ in $H^{2}\left(f_{i}, \mathbf{Q} / \mathbf{Z}\right) \subseteq \bigoplus_{i=1}^{M_{1}} H^{2}\left(Y_{i}, \mathbf{Q} / \mathbf{Z}\right)$ and $\bigoplus_{i=1}^{M_{2}} H^{2}\left(Y_{i}^{\prime}, \mathbf{Q} / \mathbf{Z}\right)$ respectively. Since we want this image to be zero, we set each of these values to zero.

$$
\begin{array}{lll} 
& \text { In } \bigoplus_{i=1}^{M_{1}} H^{2}\left(Y_{i}, \mathbf{Q} / \mathbf{Z}\right) & \text { In } \bigoplus_{i=1}^{M_{2}} H^{2}\left(Y_{i}^{\prime}, \mathbf{Q} / \mathbf{Z}\right) \\
f_{1} & b_{1}-a_{1}+a_{M_{1}+M_{2}}=0 & -c_{M_{2} / 2}+a_{1}-a_{2}=0 \\
f_{2} & -b_{1}+b_{2}=0 & -a_{1}+2 a_{2}-a_{3}=0 \\
f_{3} & -b_{2}+b_{3}=0 & -a_{2}+2 a_{3}-a_{4}=0 \\
\vdots & \vdots & \vdots \\
f_{M_{1}} & -b_{M_{1}-1}-b_{M_{1}}=0 & -a_{M_{1}-1}+2 a_{M_{1}}-a_{M_{1}+1}=0 \\
f_{M_{1}+1} & b_{M_{1}}-a_{M_{1}+1}+a_{M_{1}+2}=0 & c_{M_{2} / 2+1}-a_{M_{1}}+a_{M_{1}+1}=0 \\
f_{M_{1}+2} & a_{M_{1}+1}-2 a_{M_{1}+2}+a_{M_{1}+3}=0 & -c_{M_{2} / 2+1}+c_{M_{2} / 2+2}=0 \\
\vdots & \vdots & \vdots \\
& & -c_{M_{2}-1}-c_{M_{2}}=0 \\
& & c_{M_{2}}+c_{1}=0 \\
& \vdots & -c_{1}+c_{2}=0 \\
\vdots & \vdots &
\end{array}
$$

From this one sees that if $a_{1}, b_{1}$ and $c_{1}$ are chosen with arbitrary values, all other values are determined: by the right-hand column, one sees that

$$
\begin{gathered}
a_{2}=-c_{1}+a_{1} \\
a_{3}=-2 c_{1}+a_{1} \\
\vdots \\
a_{M_{1}+1}=-M_{1} c_{1}+a_{1}
\end{gathered}
$$

and by the left-hand column,

$$
\begin{aligned}
a_{M_{1}+M_{2}} & =-b_{1}+a_{1} \\
a_{M_{1}+M_{2}-1} & =-2 b_{1}+a_{1} \\
\vdots & \\
a_{M_{1}+1} & =-M_{2} b_{1}+a_{1},
\end{aligned}
$$

from which we see we require the additional relationship that

$$
M_{2} b_{1}=M_{1} c_{1} .
$$

If this relationship is fulfilled, then all the equations are satisfied. Thus

$$
H^{2}\left(X, \mathbf{G}_{m}\right)=\left\{(a, b, c) \mid a, b, c \in \mathbf{Q} / \mathbf{Z} \text { and } M_{2} b=M_{1} c\right\} .
$$


A similar but much simpler calculation shows that

$$
H^{2}\left(S, \mathbf{G}_{m}\right)=\mathbf{Q} / \mathbf{Z},
$$

and the map $H^{2}\left(S, \mathbf{G}_{m}\right) \rightarrow H^{2}\left(X, \mathbf{G}_{m}\right)$ is given by $a \mapsto(a, 0,0)$. This proves the theorem in this case.

In the odd case, one gets a very similar table, and one finds that

$$
\begin{aligned}
a_{2} & =-c_{1}+a_{1} \\
a_{3} & =-2 c_{1}+a_{1} \\
\vdots & \\
a_{\left(M_{1}+1\right) / 2} & =-\frac{M_{1}-1}{2} c_{1}+a_{1} \\
a_{\left(M_{1}+3\right) / 2} & =-M_{1} c_{1}+2 a_{1} \\
a_{\left(M_{1}+5\right) / 2} & =-\frac{M_{1}+1}{2} c_{1}+a_{1} \\
\vdots & \\
a_{M_{1}+2} & =-M_{1} c_{1}+a_{1}
\end{aligned}
$$

(which reduces to

$$
\begin{aligned}
& a_{2}=-c_{1}+2 a_{1} \\
& a_{3}=-c_{1}+a_{1}
\end{aligned}
$$

if $\left.M_{1}=1,\right)$ and

$$
\begin{gathered}
a_{M_{1}+M_{2}+1}=-b_{1}+a_{1} \\
\vdots \\
a_{M_{1}+2}=-M_{2} b_{1}+a_{1},
\end{gathered}
$$

and we obtain similar results.

We now move to the global situation. The next case to consider is that $Z$ is a non-singular curve contained in the discriminant locus $\Sigma$, and such that the fibre over a point of $Z$ is of type $I_{N}$.

Theorem 2.10. Let $Z \subseteq S$ be a smooth component of $\Sigma$ of fibre type $I_{M}$ which does not intersect any other component of $\Sigma$. Then if $Z$ is case I (see §1), then

$$
H_{Z}^{2}\left(S, i_{*} A\right)=\mathbf{Q} / \mathbf{Z}
$$

if $Z$ is case $I^{*}$, then

$$
H_{Z}^{2}\left(S, i_{*} A\right)=\mathbf{Z} / 2 \mathbf{Z}
$$

Proof. First note that $\underline{H}_{Z}^{2}(S, \mathcal{E})=\underline{H}_{Z}^{3}(S, \mathcal{E})=0$, since $Z$ intersects no other component of $\Sigma$. Thus $\underline{H}_{Z}^{2}\left(S, i_{*} A\right)=\underline{H}_{Z}^{2}\left(S, P_{X / S}\right)$ by Prop. $\left.2.2 \mathrm{c}\right)$. Write $f^{-1}(Z)$ as a union of components $Y_{1}, \ldots, Y_{M^{\prime}}$ if we are in Case $I$ and $Y_{1}, \ldots, Y_{M^{\prime} / 2+1}$ if we are in Case $I^{*}$. ( $M^{\prime}=M$ if $M$ is even, $M^{\prime}=M+1$ if $M$ is odd.) Applying Prop. 1.6, one obtains an exact sequence

$$
0 \rightarrow \underline{H}_{Z}^{2}\left(S, i_{*} A\right) \rightarrow \bigoplus_{i<j} k_{*}(\mathbf{Q} / \mathbf{Z})_{Y_{i} \cap Y_{j}} \rightarrow \bigoplus_{k} R^{2} k_{*}(\mathbf{Q} / \mathbf{Z})_{Y_{i}}
$$


Taking global sections gives us a sequence

$$
0 \rightarrow H_{Z}^{2}\left(S, i_{*} A\right) \rightarrow \bigoplus_{i<j} H^{0}\left(Y_{i} \cap Y_{j}, \mathbf{Q} / \mathbf{Z}\right) \stackrel{d}{\longrightarrow} \bigoplus_{i} \mathbf{Q} / \mathbf{Z} .
$$

In Case $I$, the matrix of $d$ is given by

$$
\begin{aligned}
& \begin{array}{llll}
C_{1} & C_{2} & \cdots & C_{M^{\prime}}
\end{array}
\end{aligned}
$$

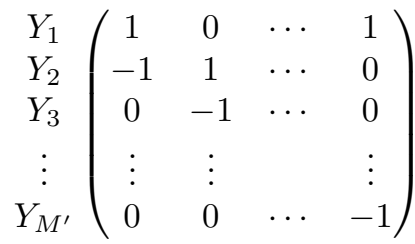

where $C_{i}=Y_{i} \cap Y_{i+1}, C_{M^{\prime}}=Y_{1} \cap Y_{M^{\prime}}$. The kernel of this map is clearly $\mathbf{Q} / \mathbf{Z}$. In the case $I^{*}$, the matrix is

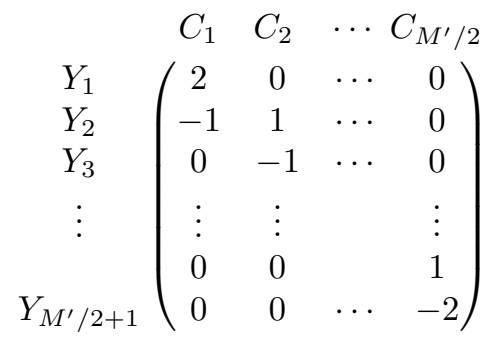

where $C_{i}=Y_{i} \cap Y_{i+1}$. Here, the kernel is clearly $\mathbf{Z} / 2 \mathbf{Z}$. •

We can now put all of our calculations together:

Theorem 2.11. Let $Z \subseteq S$ consist of a union of a finite number of isolated points $P_{1}, \ldots, P_{n_{1}}$, a finite number of non-singular curves $Z_{1}, \ldots, Z_{n_{2}}$ not contained in $\Sigma$, intersecting $\Sigma$ transversally, and a finite number of components of $\Sigma$, $Z_{n_{2}+1}, \ldots, Z_{n_{4}}$ consisting of all components of $\Sigma$ of fibre type $I_{M}$ numbered so that $Z_{i}$ is Case $I$ for $n_{2}+1 \leq i \leq n_{3}$ and Case $I^{*}$ for $n_{3}+1 \leq i \leq n_{4}$. Then

$$
H_{Z}^{2}\left(S, i_{*} A\right)=\bigoplus_{i=1}^{n_{1}} H_{P_{i}}^{2}\left(S, i_{*} A\right) \oplus \bigoplus_{i=1}^{n_{2}} M W\left(f^{-1}\left(Z_{i}\right) / Z_{i}\right)_{\text {tors }} \oplus G,
$$

where

1)

$$
H_{P_{i}}^{2}\left(S, i_{*} A\right)= \begin{cases}\mathbf{Z} / 2 \mathbf{Z} & \text { if } P_{i} \text { is a collision point of type } I I I+I_{0}^{*}, \\ 0 & \text { otherwise. }\end{cases}
$$

2) There is an exact sequence

$$
0 \rightarrow(\mathbf{Z} / 2 \mathbf{Z})^{N^{*}} \rightarrow G \rightarrow(\mathbf{Q} / \mathbf{Z})^{n_{3}-n_{2}} \oplus\left(\frac{1}{2} \mathbf{Z} / \mathbf{Z}\right)^{n_{4}-n_{3}} \stackrel{\alpha}{\longrightarrow}(\mathbf{Q} / \mathbf{Z})^{N},
$$

where $N^{*}$ is the number of collision points of type $I_{a}+I_{b}^{*}$ with $a>0$, a even, and $N$ is the number of collision points of type $I_{a}+I_{b}$. Denoting these latter collision points $Q_{1}, \ldots, Q_{n}$, the map $\alpha$ is defined by

$$
\left(\alpha\left(a_{n_{2}+1}, \ldots, a_{n_{4}}\right)\right)_{i}=a_{j} M_{k}-a_{k} M_{j},
$$

if $Q_{i}$ is at the collision of $Z_{j}$ and $Z_{k}$, of fibre type $I_{M_{j}}$ and $I_{M_{k}}$ respectively. 
Proof. Let

$$
Z^{\prime}=\left\{Q_{1}, \ldots, Q_{N}, Q_{1}^{*}, \ldots, Q_{N^{\prime}}^{*}\right\} \cup\left(\left(Z_{1} \cup \cdots \cup Z_{n_{2}}\right) \cap\left(Z_{n_{2}+1} \cup \cdots \cup Z_{n_{4}}\right)\right),
$$

the $Q_{i}^{*}$ being the collision points of type $I_{a}+I_{b}^{*}$. As in Proposition 2.8, we have the sequence

$$
\begin{aligned}
0=H_{Z-Z^{\prime}}^{1}\left(S-Z^{\prime}, i_{*} A\right) \rightarrow H_{Z^{\prime}}^{2}\left(S, i_{*} A\right) \rightarrow & H_{Z}^{2}\left(S, i_{*} A\right) \\
& \rightarrow H_{Z-Z^{\prime}}^{2}\left(S-Z^{\prime}, i_{*} A\right) \rightarrow H_{Z^{\prime}}^{3}\left(S, i_{*} A\right)
\end{aligned}
$$

which yields

$$
\begin{aligned}
& 0 \rightarrow(\mathbf{Z} / 2 \mathbf{Z})^{N^{*}} \rightarrow H_{Z}^{2}\left(S, i_{*} A\right) \\
& \rightarrow \bigoplus_{i=1}^{n_{1}} H_{P_{i}}^{2}\left(S, i_{*} A\right) \oplus \bigoplus_{i=1}^{n_{2}} M W\left(f^{-1}\left(Z_{i}\right) / Z_{i}\right)_{\text {tors }} \oplus(\mathbf{Q} / \mathbf{Z})^{n_{3}-n_{2}} \oplus\left(\frac{1}{2} \mathbf{Z} / \mathbf{Z}\right)^{n_{4}-n_{3}} \\
& \rightarrow(\mathbf{Q} / \mathbf{Z})^{N}
\end{aligned}
$$

The part $\bigoplus_{i=1}^{n_{1}} H_{P_{i}}^{2}\left(S, i_{*} A\right) \oplus \bigoplus_{i=1}^{n_{2}} M W\left(f^{-1}\left(Z_{i}\right) / Z_{i}\right)_{t o r s}$ clearly factors out of this sequence, leaving the exact sequence (2.2). Using Theorem 2.9, one sees that the map $\alpha$ can be written as described.

Remark 2.12. Note that if $s \in S$, there is a natural map

$$
H_{Z}^{2}\left(S, i_{*} A\right) \stackrel{\cong}{\longrightarrow} H^{0}\left(S, \underline{H}_{Z}^{2}\left(S, i_{*} A\right)\right) \longrightarrow\left(\underline{H}_{Z}^{2}\left(S, i_{*} A\right)\right)_{\bar{s}} \subseteq W C\left(A_{\bar{s}}\right) .
$$

If $\gamma \in H_{Z}^{2}\left(S, i_{*} A\right)$, and $t \in S^{(1)}$ is the generic point of a curve $C$, then the invariant of $\gamma$ along $C$ is the image of $\gamma$ in $W C\left(A_{\bar{t}}\right)=(\mathbf{Q} / \mathbf{Z})^{r}, r=2,1$ or 0 depending on whether the geometric fibre $X_{\bar{t}}$ is non-singular, multiplicative $\left(I_{M}, M>0\right)$ or additive (any other singular Kodaira fibre-type).

In Proposition 2.11, given $\gamma \in H_{Z}^{2}\left(S, i_{*} A\right)$, the invariant of $\gamma$ along $Z_{i}$ is the image of $\gamma$ under the natural projection $H_{Z}^{2}\left(S, i_{*} A\right) \rightarrow M W\left(f^{-1}\left(Z_{i}\right) / Z_{i}\right)_{\text {tors }} \subseteq(\mathbf{Q} / \mathbf{Z})^{\oplus 2}$ if $1 \leq i \leq n_{2}$, and the image of $\gamma$ under the natural projection $H_{Z}^{2}\left(S, i_{*} A\right) \rightarrow G \rightarrow$ $\mathbf{Q} / \mathbf{Z}$ if $n_{2}+1 \leq i \leq n_{4}$.

If $\gamma$ maps to 0 in $H^{2}\left(S, i_{*} A\right)$, then there exists an element $E \in W C(A)$ which realises these invariants. If one finds a relatively minimal model $f^{\prime}: X^{\prime} \rightarrow S$ for $E$ with $X_{\eta}^{\prime}=E$, and if $t \in S^{(1)}$ is the generic point of a curve $C \subseteq S$, then $X^{\prime}(\bar{t}) \rightarrow \operatorname{Spec} \mathcal{O}_{S, \bar{t}}$ (here $\left.X^{\prime}(\bar{t})=X^{\prime} \times{ }_{S} \operatorname{Spec} \mathcal{O}_{S, \bar{t}}\right)$ is relatively minimal, and hence the geometric fibre $X_{\bar{t}}^{\prime}$ is of Kodaira fibre type ${ }_{m} I_{n}$, where $m$ is the order of the invariant of $\gamma$ along $C$, with $X_{\bar{t}}$ being of fibre type $I_{n}$.

Finally, note that there is no need to consider $Z \subseteq S$ in Proposition 2.11 containing other components of $\Sigma$, not of fibre type $I_{m}$, as there would be no additional contribution to $H_{Z}^{2}\left(S, i_{*} A\right)$, as $W C\left(A_{\bar{t}}\right)=0$ if $t \in S^{(1)}$ and $X_{\bar{t}}$ not of type $I_{m}$, $m \geq 0$.

Example 2.13. We shall introduce here four examples which we shall come back to several times later on.

1) Take any elliptic curve $E$, and put $X_{1}=\mathbf{P}^{2} \times E, f_{1}: X_{1} \rightarrow \mathbf{P}^{2}$ the first projection. If $Z=Z_{1} \cup \cdots \cup Z_{n}$ is a union of non-singular, irreducible curves, then $H_{Z_{i}}^{2}\left(\mathbf{P}^{2}, i_{*} A\right)=(\operatorname{Pic} E)_{\text {tors }}, H_{Z}^{2}\left(\mathbf{P}^{2}, i_{*} A\right)=(\operatorname{Pic} E)_{\text {tors }}^{\oplus n}$. Of course, $(\operatorname{Pic} E)_{\text {tors }}=$ $(\mathbf{Q} / \mathbf{Z})^{\oplus 2}$. (We do not actually need the $Z_{i}$ to be non-singular.) 


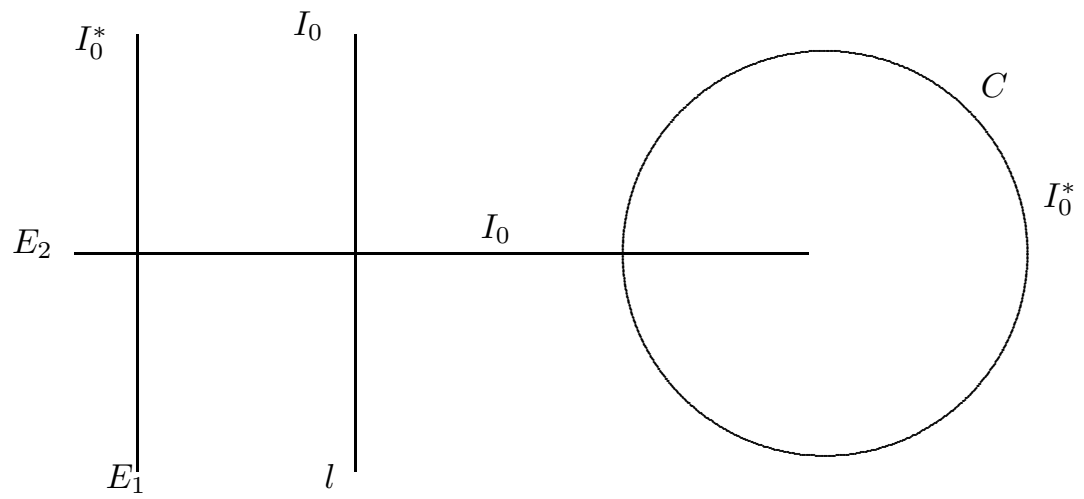

FiguRe 2.1

2) Let $p_{1}$ and $p_{2}$ be the projections of $\mathbf{P}^{2} \times \mathbf{P}^{1}$ onto $\mathbf{P}^{2}$ and $\mathbf{P}^{1}$ respectively. Let $B \subseteq \mathbf{P}^{2} \times \mathbf{P}^{1}$ be $p_{1}^{-1}(C) \cup p_{2}^{-1}\left\{P_{1}, P_{2}, P_{3}, P_{4}\right\}$ with $C \subseteq \mathbf{P}^{2}$ a smooth conic and $P_{1}, \ldots, P_{4} \in \mathbf{P}^{1}$ four distinct points. Consider the double cover of $\mathbf{P}^{2} \times \mathbf{P}^{1}$ branched over $B$. This has a singular locus, consisting of four curves, which can be resolved by blowing those curves up once, and we obtain in this way an elliptic fibration $f_{2}: X_{2} \rightarrow \mathbf{P}^{2}$ induced by the first projection. This has $\Sigma=C$, of fibre type $I_{0}^{*}$.

If $Z_{1} \subseteq \mathbf{P}^{2}$ is a non-singular curve intersecting $C$ transversally, it is not hard to see that $M W\left(f_{2}^{-1}\left(Z_{1}\right) / Z_{1}\right)_{\text {tors }}=\mathbf{Z} / 2 \mathbf{Z} \oplus \mathbf{Z} / 2 \mathbf{Z}$. Thus this group is the value of $H_{Z_{1}}^{2}\left(\mathbf{P}^{2}, i_{*} A\right)$.

Let $l$ be a line tangent to $C$. Here we blow up $\mathbf{P}^{2}$ twice to get a fibration $f_{2}^{\prime}$ : $X_{2}^{\prime} \rightarrow S_{2}$ to obtain a picture on $S_{2}$ as shown in Figure 2.1. Put $Z_{2}=l \cup E_{1} \cup E_{2}$. We see that $f_{2}^{\prime-1}(l)$ is a trivial elliptic fibration over $l$, and thus

$$
\begin{aligned}
H_{Z_{2}}^{2}\left(S_{2}, i_{*} A\right) & =M W\left(f_{2}^{-1}(l) / l\right)_{\text {tors }} \oplus M W\left(f_{2}^{\prime-1}\left(E_{2}\right) / E_{2}\right)_{\text {tors }} \\
& =(\mathbf{Q} / \mathbf{Z})^{\oplus 2} \oplus \mathbf{Z} / 2 \mathbf{Z} \oplus \mathbf{Z} / 2 \mathbf{Z} .
\end{aligned}
$$

3) Take a general net of quadrics in $\mathbf{P}^{3}$, blow up the eight base-points to obtain $X$, and thus obtain a morphism $X \rightarrow \mathbf{P}^{2}$. The discriminant locus $\Sigma$ is the dual curve to the quartic of singular quadrics in the net and thus has 24 cusps and 28 nodes. Of course $\amalg_{\mathbf{P}^{2}}(A)=0$; we want to know what $\amalg_{\mathbf{P}^{2}-\Sigma}(A)$ is.

To discover this, we first blow up $\mathbf{P}^{2}$ to obtain $\Sigma$ s.n.c. A node gets blown up once to obtain Figure 2.2 while a cusp must be blown up three times (see [11] for details), to obtain Figure 2.3. We see the curve of fibre type $I_{1}$ is case $I^{*}$. Let $f_{3}: X_{3} \rightarrow S$ be the resulting modified Miranda model, and let $Z=\Sigma$. Then

$$
H_{Z}^{2}\left(S, i_{*} A\right)=(\mathbf{Z} / 2 \mathbf{Z})^{\oplus 24} \oplus \mathbf{Z} / 2 \mathbf{Z} .
$$

The first term comes from the $24 I_{0}^{*}+I I I$ collisions, the second along the curve of type $I_{1}$. There can be no multiple fibres over the curves of fibre type $I_{2}$.

4) Consider over $\mathbf{P}^{2}$ with coordinates $(x: y: z)$ the Weierstrass model obtained by the equation

$$
s^{2}=t^{3}+x y z^{2} t+x y z^{3}(x-y) / \sqrt{27}
$$




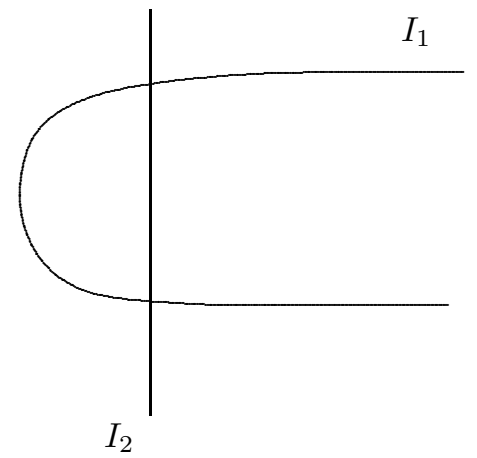

Figure 2.2

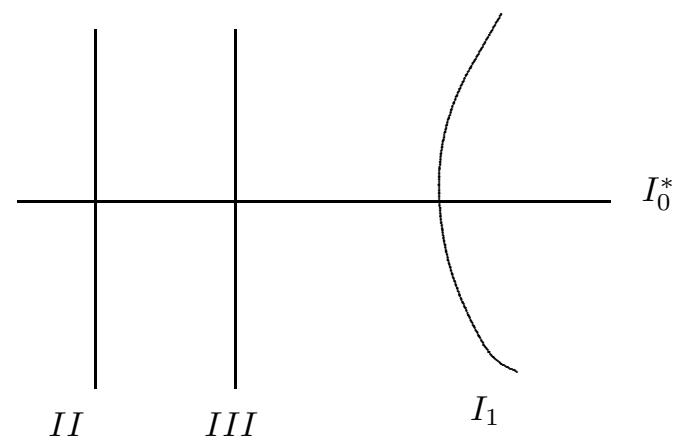

FiguRE 2.3

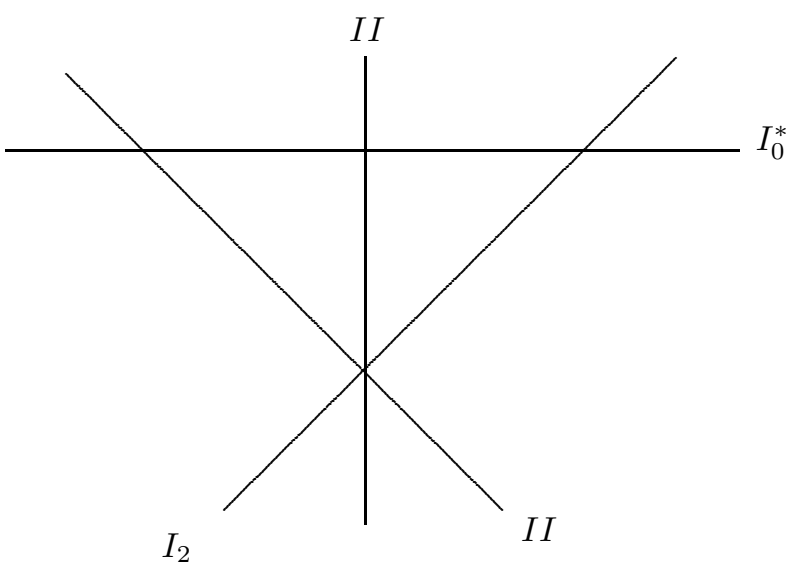

FiguRE 2.4

This has discriminant curve given by the equation

$$
x^{2} y^{2} z^{6}(x+y)^{2}=0,
$$

which looks like Figure 2.4. Blowing up the point $(0: 0: 1)$, we obtain a Weierstrass 


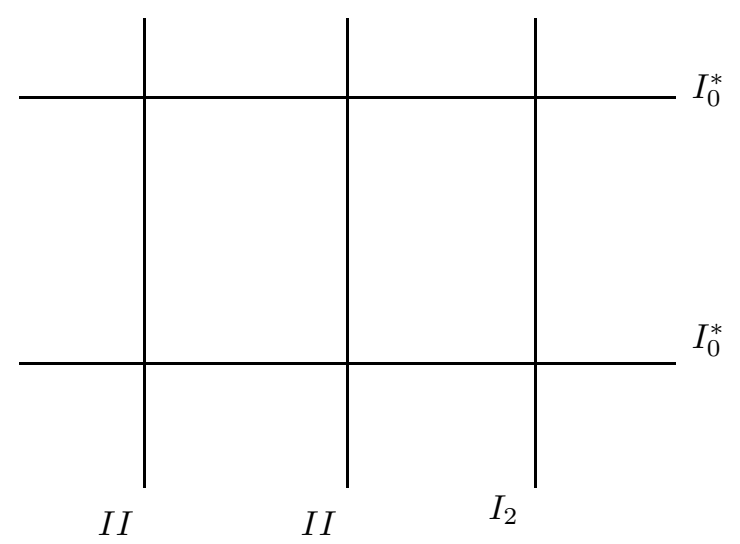

FiguRE 2.5

model over $F_{1}$ with discriminant curve as shown in Figure 2.5. Let $f_{4}: X_{4} \rightarrow F_{1}$ be the Miranda model of this Weierstrass fibration, and let $Z$ be the curve of fibre type $I_{2}$. Then $H_{Z}^{2}\left(S, i_{*} A\right)=\mathbf{Z} / 2 \mathbf{Z} \oplus \mathbf{Z} / 2 \mathbf{Z} \oplus \mathbf{Z} / 2 \mathbf{Z}$. We have the possibility of multiplicity 2 fibres along $Z$ or isolated multiple fibres at $P_{1}$ or $P_{2}$. (We will show in Theorem 3.5 that this is the appropriate extension of $\mathbf{Z} / 2 \mathbf{Z}$ by $\mathbf{Z} / 2 \mathbf{Z} \oplus \mathbf{Z} / 2 \mathbf{Z}$.)

Remark 2.14. The reader may note that in the calculations of Propositions 2.62.10, we $\operatorname{did}$ not need to assume that $\operatorname{dim} X=3, \operatorname{dim} S=2$, so long as we had $f: X \rightarrow S$ satisfying our hypothesis. If one could prove without the use of a model that $H_{Z}^{2}\left(S, i_{*} A\right)=H_{Z}^{3}\left(S, i_{*} A\right)=0$ for all $Z \subseteq S$ of codimension $\geq 3$, then one can remove codimension 3 sets without changing the results, and thus extend the results of this section to all dimensions, as [16] can always give a good model over an open set $U \subseteq S$ with $S-U$ codimension $\geq 3$.

\section{The FIRST OBSTRUCTION}

Our next goal is to understand the map $H_{Z}^{2}\left(S, i_{*} A\right) \rightarrow H^{2}\left(S, i_{*} A\right)$; in particular, we wish to know its kernel. We have (Theorem 1.1) an inclusion

$$
0 \rightarrow H^{2}\left(S, i_{*} A\right) \rightarrow H^{2}\left(S, i_{*} i^{*} P_{X / S}\right)
$$

and an exact sequence

$$
0 \rightarrow G(X / S) \rightarrow H^{2}\left(S, i_{*} i^{*} P_{X / S}\right) \rightarrow H^{3}(S, \mathcal{E})
$$

where

$$
G(X / S):=\operatorname{coker}\left(H^{2}(S, \mathcal{E}) \rightarrow H^{2}\left(S, P_{X / S}\right)\right) .
$$

We will first study the composed map

$$
H_{Z}^{2}\left(S, i_{*} A\right) \stackrel{\phi}{\longrightarrow} H^{3}(S, \mathcal{E}),
$$

which we call the first obstruction, and then the induced map

$$
\operatorname{ker} \phi \stackrel{\psi}{\longrightarrow} G(X / S)
$$

which we call the second obstruction. We then have

$$
\frac{\amalg_{S-Z}(A)}{\amalg_{S}(A)}=\operatorname{ker} \psi \text {. }
$$


The map $\phi: H_{Z}^{2}\left(S, i_{*} A\right) \rightarrow H^{3}(S, \mathcal{E})$ is a composition of the maps

$$
H_{Z}^{2}\left(S, i_{*} A\right) \cong H_{Z}^{2}\left(S, i_{*} i^{*} P_{X / S}\right) \stackrel{\phi_{1}}{\longrightarrow} H_{Z}^{3}(S, \mathcal{E}) \stackrel{\phi_{2}}{\longrightarrow} H^{3}(S, \mathcal{E}),
$$

the first isomorphism by $(1.2)$, as $H_{Z}^{2}(S, \mathbf{Z})=H_{Z}^{1}(S, \mathbf{Q} / \mathbf{Z})=0$.

We will first clarify exactly what $H_{Z}^{3}(S, \mathcal{E})$ and the map $\phi_{2}$ are. Recall from $[8$, 1.8] that, since we are assuming that all components of $\Sigma$ are normal,

$$
\mathcal{E}=\bigoplus_{t \in S^{(1)}} \mathcal{E}_{t}
$$

where $\mathcal{E}_{t}=i_{t *} i_{t}^{*} \mathcal{E}$, with $i_{t}: t \rightarrow S$ the inclusion. Furthermore, we have an exact sequence

$$
0 \rightarrow j_{t *} \mathbf{Z}_{C(t)} \rightarrow j_{t *} q_{t *} \mathbf{Z}_{\bar{C}(t)} \rightarrow \mathcal{E}_{t} \rightarrow 0,
$$

where $j_{t}: C(t) \rightarrow S$ is the inclusion and $q_{t}: \bar{C}(t) \rightarrow C(t)$ the natural projection. The first map is given by $a \mapsto\left(m_{i} a\right)$ where $m_{i}$ are the multiplicities of the components $X_{t}^{i}$ of $X_{t}$. From this, one sees easily

\section{Proposition 3.1.}

1) If $t \notin Z$, then

$$
H_{Z}^{3}\left(S, \mathcal{E}_{t}\right)=\operatorname{coker}\left(H^{0}(C(t) \cap Z, \mathbf{Q} / \mathbf{Z}) \rightarrow H^{0}\left(q_{t}^{-1}(C(t) \cap Z), \mathbf{Q} / \mathbf{Z}\right)\right)
$$

where the map is given as follows. If $Q \in C(t) \cap Z, q_{t}^{-1}(Q)=\left\{P_{1}, \ldots, P_{n}\right\}$, with $P_{i}$ in $C\left(t_{j_{i}}\right)$, then the map for $a \in H^{0}(Q, \mathbf{Q} / \mathbf{Z})$ is

$$
a \mapsto\left(r_{i} m_{j_{i}} a\right)
$$

where $r_{i}$ is the ramification of $C\left(t_{j_{i}}\right) \rightarrow C(t)$ at $P_{i}$ and $m_{j_{i}}$ is the multiplicity of the component $X_{t}^{j_{i}}$. The map $\phi_{2}: H_{Z}^{3}\left(S, \mathcal{E}_{t}\right) \rightarrow H^{3}\left(S, \mathcal{E}_{t}\right)$ is given by the diagram

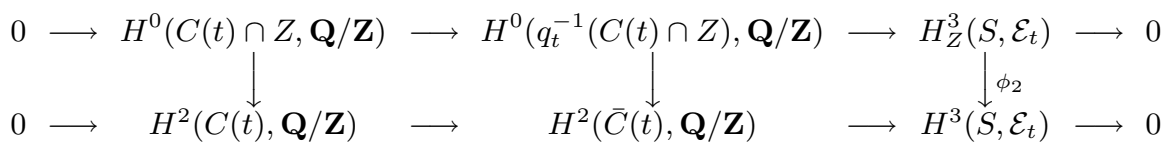

where the first and second vertical arrows are the natural Gysin maps.

2) If $t \in Z$, then $H_{Z}^{3}\left(S, \mathcal{E}_{t}\right) \longrightarrow H^{3}\left(S, \mathcal{E}_{t}\right)$ is an isomorphism.

We can describe $\operatorname{ker} \phi$ as

$$
\operatorname{ker}\left(H_{Z}^{2}\left(S, i_{*} A\right) \stackrel{\phi}{\longrightarrow} \bigoplus_{t \in S^{(1)}} H^{3}\left(S, \mathcal{E}_{t}\right)\right)=\bigcap_{t \in S^{(1)}} \operatorname{ker}\left(H_{Z}^{2}\left(S, i_{*} A\right) \stackrel{\phi_{t}}{\longrightarrow} H^{3}\left(S, \mathcal{E}_{t}\right)\right),
$$

so we can treat each $t \in \Sigma$ separately.

By Theorem 2.11, we can split the study of $\phi_{t}$ into three cases:

(1) Describe $\phi_{t}: H_{P}^{2}\left(S, i_{*} A\right) \rightarrow H^{3}\left(S, \mathcal{E}_{t}\right)$ for $P$ a collision point of type $I I I+I_{0}^{*}$. Here the only values for $t$ we need to consider are the generic points of the two components of $\Sigma$ meeting at $P$.

(2) Describe $\phi_{t}: H_{Z}^{2}\left(S, i_{*} A\right) \rightarrow H^{3}\left(S, \mathcal{E}_{t}\right)$, where $Z$ is a curve transversal to $\Sigma$. Here we are interested in those $t$ which are generic points of components of $\Sigma$ which $Z$ intersects.

(3) Describe $\phi_{t}: H_{Z}^{2}\left(S, i_{*} A\right) \rightarrow H^{3}\left(S, \mathcal{E}_{t}\right)$ when $Z \subseteq \Sigma$ consists of all components of $\Sigma$ of type $I_{M}, M \geq 1$. 
First, suppose that $P$ is an isolated point of $Z$, at a collision point of type $I_{0}^{*}+I I I$, with generic point $t$ of the curve of fibre type $I_{0}^{*}$ and $t^{\prime}$ of the curve of fibre type $I I I$. The map $H_{P}^{2}\left(S, i_{*} A\right) \rightarrow H_{P}^{3}(S, \mathcal{E})$ corresponds exactly to the map $H^{1}\left(S, i_{*} A\right) \rightarrow H^{2}(S, \mathcal{E})$ in the local situation in Theorem 2.3. This map has been calculated for this example in Theorem 2.4:

$$
H_{P}^{3}(S, \mathcal{E})=\operatorname{coker}\left(\mathbf{Q} / \mathbf{Z} \stackrel{\alpha_{1}}{\longrightarrow}(\mathbf{Q} / \mathbf{Z})^{4}\right) \oplus \operatorname{coker}\left(\mathbf{Q} / \mathbf{Z} \stackrel{\alpha_{2}}{\longrightarrow}(\mathbf{Q} / \mathbf{Z})^{2}\right),
$$

where by Prop. $3.1 \alpha_{1}$ is given by

$$
a \mapsto(a, 2 a, 2 a, a)
$$

and $\alpha_{2}$ by

$$
a \mapsto(a, a),
$$

with components corresponding to the inverse image of $P$ on curves $C\left(t_{1}\right), \ldots, C\left(t_{4}\right)$ and $C\left(t_{1}^{\prime}\right), C\left(t_{2}^{\prime}\right)$ over which the normalizations of $Y_{1}, \ldots, Y_{4}$ and $Y_{1}^{\prime}, Y_{2}^{\prime}$ are fibred. (Here we adopt the notation of the proof of Theorem 2.4.) We have $q_{t}^{-1}(P)=$ $\left\{P_{1}, \ldots, P_{4}\right\}, P_{i} \in C\left(t_{i}\right), q_{t^{\prime}}^{-1}(P)=\left\{P_{1}^{\prime}, P_{2}^{\prime}\right\}, P_{i}^{\prime} \in C\left(t_{i}^{\prime}\right)$. In this case, $H_{P}^{2}\left(S, i_{*} A\right)=$ $\mathbf{Z} / 2 \mathbf{Z}$, and writing the non-zero element of $\mathbf{Z} / 2 \mathbf{Z} \subseteq \mathbf{Q} / \mathbf{Z}$ as $1 / 2$, we have $\phi_{1}(1 / 2) \in$ $H_{P}^{3}(S, \mathcal{E})$ is represented by the element which was given by $(2.1)$. Writing down the value of this representative on each point, we get:

$$
\begin{array}{cc}
P_{1}: 0 & P_{1}^{\prime}: 0 \\
P_{2}: 1 / 2 & P_{2}^{\prime}: 1 / 2 \\
P_{3}: 0 & \\
P_{4}: 1 / 2 &
\end{array}
$$

This describes completely the map $\phi_{1}: H_{P}^{2}\left(S, i_{*} A\right) \rightarrow H_{P}^{3}(S, \mathcal{E})$, and we compose this with $\phi_{2}$ to obtain $\phi$ in this case.

Next consider a curve $C \subseteq Z$ transversal to $\Sigma$. The calculation is easiest if we assume $C$ is complete.

Lemma 3.2. Let $f: Y \rightarrow C$ be an elliptic surface with a section and at least one singular fibre, $C$ a smooth, irreducible, complete curve. Then $f^{*}: H^{1}(C, \mathbf{Q} / \mathbf{Z}) \rightarrow$ $H^{1}(Y, \mathbf{Q} / \mathbf{Z})$ is an isomorphism.

Proof. $f^{*}$ is clearly injective. Now $H^{1}(C, \mathbf{Q} / \mathbf{Z})=(\operatorname{Pic} C)_{\text {tors }}$ and $H^{1}(X, \mathbf{Q} / \mathbf{Z})=$ $(\operatorname{Pic} X)_{\text {tors }}$, so by [4], Cor. 5.2.2, $\operatorname{coker}\left(f^{*}\right)=N S(X)_{\text {tors }}$. But this is zero, say by [6] (1.48).

Proposition 3.3. Let $C \subseteq S$ be a smooth irreducible complete curve meeting $\Sigma$ transversally, $Y=f^{-1}(C)$ a smooth surface. Then the map $\phi_{1}: H_{C}^{2}\left(S, i_{*} A\right) \rightarrow$ $H_{C}^{3}(S, \mathcal{E})$ is given as follows: if $\sigma \in{ }_{m} M W(Y / C)={ }_{m} H_{C}^{2}\left(S, i_{*} A\right), \sigma_{0}$ the zero section, then on $Y$ one can write $m\left(\sigma-\sigma_{0}\right) \sim E, E$ a divisor supported in the fibres of $f: Y \rightarrow C . E \otimes(1 / m)$ then gives the appropriate element of $H_{C}^{3}(S, \mathcal{E})$, identifying $H_{C}^{3}(S, \mathcal{E})$ in the natural way with $\left((\operatorname{Pic} Y)_{f i b} / f^{*} \operatorname{Pic} C\right) \otimes \mathbf{Q} / \mathbf{Z}$, where $(\operatorname{Pic} Y)_{f i b} \subseteq \operatorname{Pic} Y$ is the subgroup of divisors supported on fibres of $f$.

Proof. We have an exact sequence

$$
0 \rightarrow H_{C}^{2}\left(S, P_{X / S}\right) \rightarrow H_{C}^{2}\left(S, i_{*} A\right) \rightarrow H_{C}^{3}(S, \mathcal{E}) \rightarrow H_{C}^{3}\left(S, P_{X / S}\right) .
$$

By Propositions 1.4, 1.5, 3.1 and Lemma 3.2,

$$
H_{C}^{2}\left(S, P_{X / S}\right)=H^{1}(Y, \mathbf{Q} / \mathbf{Z}) / H^{1}(C, \mathbf{Q} / \mathbf{Z})=0,
$$




$$
H_{C}^{3}\left(S, P_{X / S}\right)=H^{2}(Y, \mathbf{Q} / \mathbf{Z}) / H^{2}(C, \mathbf{Q} / \mathbf{Z})
$$

and

$$
H_{C}^{3}(S, \mathcal{E})=\bigoplus_{1 \leq i \leq n} \frac{(\mathbf{Q} / \mathbf{Z})^{c\left(f_{i}\right)}}{\mathbf{Q} / \mathbf{Z}}=\left((\operatorname{Pic} Y)_{f i b} / f^{*} \operatorname{Pic} C\right) \otimes \mathbf{Q} / \mathbf{Z}
$$

where $f_{i}, 1 \leq i \leq n$, runs over the reducible fibres of $f: Y=f^{-1}(C) \rightarrow C$, and $c\left(f_{i}\right)$ is the number of components of $f_{i}$. The map

$$
H_{C}^{3}(S, \mathcal{E}) \rightarrow H_{C}^{3}\left(S, P_{X / S}\right)
$$

is clearly the Gysin map.

Now let $U \subseteq C$ be the largest open subset such that $\left.f\right|_{f^{-1}(U)}$ is smooth, $C-U$ consisting of $n$ points. Then there is a commutative diagram

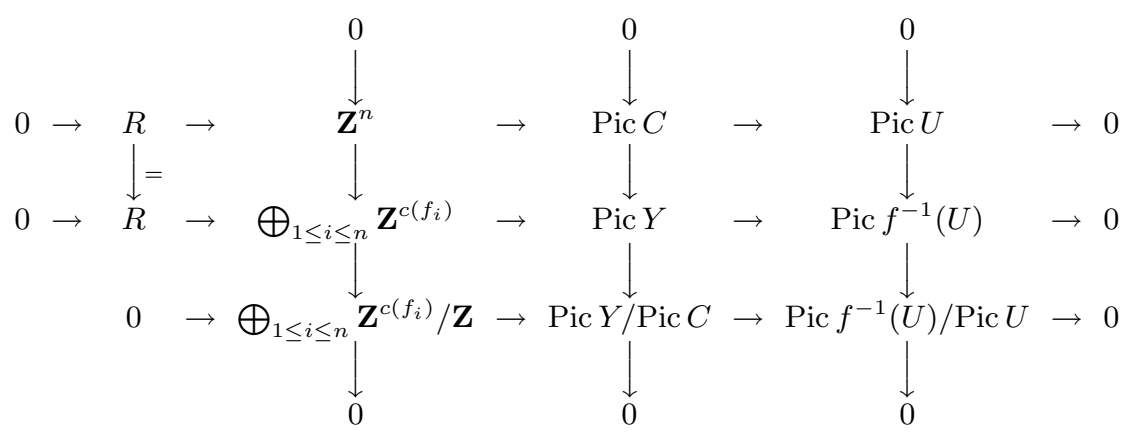

the equality because there are no relations on divisors on $Y$ supported in the singular fibres other than those which come from $C$.

Applying the torsion functor to the bottom row, one obtains

$$
0 \rightarrow\left(\frac{\operatorname{Pic} f^{-1}(U)}{\operatorname{Pic} U}\right)_{\text {tors }} \stackrel{\partial}{\longrightarrow} \bigoplus_{1 \leq i \leq n} \frac{(\mathbf{Q} / \mathbf{Z})^{c\left(f_{i}\right)}}{\mathbf{Q} / \mathbf{Z}} \stackrel{\alpha_{1}}{\longrightarrow} \frac{(\operatorname{Pic} Y) \otimes \mathbf{Q} / \mathbf{Z}}{(\operatorname{Pic} C) \otimes \mathbf{Q} / \mathbf{Z}}
$$

The first term is $M W(Y / C)_{\text {tors }}$ by the proof of 2.7 , and there is a natural injective map

$$
\frac{(\operatorname{Pic} Y) \otimes \mathbf{Q} / \mathbf{Z}}{(\operatorname{Pic} C) \otimes \mathbf{Q} / \mathbf{Z}} \stackrel{\alpha_{2}}{\longrightarrow} \frac{H^{2}(Y, \mathbf{Q} / \mathbf{Z})}{H^{2}(C, \mathbf{Q} / \mathbf{Z})},
$$

by (1.3). $\alpha_{2} \circ \alpha_{1}$ clearly coincides with the map

$$
H_{C}^{3}(S, \mathcal{E}) \rightarrow H_{C}^{3}\left(S, P_{X / S}\right) .
$$

Thus we have seen in another way that $H_{C}^{2}\left(S, i_{*} A\right)=M W(Y / C)_{t o r s}$, but the point is that now we can interpret the map

$$
H_{C}^{2}\left(S, i_{*} A\right) \rightarrow H_{C}^{3}(S, \mathcal{E})
$$

as the map $\partial$ in (3.2). In the long exact sequence associated with the torsion functor, this map is defined as follows. If

$$
0 \rightarrow A \rightarrow B \rightarrow C \rightarrow 0
$$

is an exact sequence of groups, one obtains a long exact sequence

$$
0 \rightarrow A_{\text {tors }} \rightarrow B_{\text {tors }} \rightarrow C_{\text {tors }} \stackrel{\partial}{\longrightarrow} A \otimes \mathbf{Q} / \mathbf{Z} .
$$


If $c \in C_{\text {tors }}$ with $m c=0$, then $c$ is the image of an element $b \in B$ with $m b=a \in A$. Then $\partial(c)=a \otimes(1 / m)$. This agrees with the description given in the statement of the theorem.

The values for the map described in Theorem 3.3 are well-known: see, for example, [6] for a complete table of values.

We move on to those components of $Z$ contained in $\Sigma$. All details of the global calculations are given in the appendix; here we just state the results and perform a local calculation.

Theorem 3.4. Let $C \subseteq Z$ be a complete curve of fibre type $I_{M}$ and case $I$, with generic point $t$. We have

$$
\begin{gathered}
H_{Z}^{3}\left(S, \mathcal{E}_{t}\right)=H^{3}\left(S, \mathcal{E}_{t}\right)=\operatorname{coker}\left(\mathbf{Q} / \mathbf{Z} \rightarrow \bigoplus_{i=1}^{M^{\prime}} \mathbf{Q} / \mathbf{Z}\right), \\
M^{\prime}= \begin{cases}M & \text { if } M \text { is even, } \\
M+1 & D \text { if } M \text { is odd, }\end{cases}
\end{gathered}
$$

with the summands of $\bigoplus_{i=1}^{M^{\prime}} \mathbf{Q} / \mathbf{Z}$ corresponding to the components $Y_{1}, \ldots, Y_{M^{\prime}}$ over $C$ respectively. Let $\gamma \in H_{Z}^{2}\left(S, i_{*} A\right)$ have invariants $b$ along $C$ and invariants $c_{i}$ along the curves of $\Sigma$ intersecting $C$, of fibre type $I_{M_{i}}, 1 \leq i \leq s$ (see Remark 2.12 for the definition of invariants $)$. Put $\alpha=-b C^{2}-\sum_{j=1}^{s} c_{j}$. Then $\phi_{t}(\gamma) \in H^{3}\left(S, \mathcal{E}_{t}\right)$ is given by the class of the element $\beta=\left(\beta_{i}\right) \in(\mathbf{Q} / \mathbf{Z})^{M^{\prime}}$, with

$$
\beta_{i}= \begin{cases}(i-1) \alpha & \text { if } 1 \leq i \leq(M+1) / 2 \\ M \alpha & \text { if } i=(M+3) / 2 \\ (i-2) \alpha & \text { if }(M+5) / 2 \leq i \leq M+1\end{cases}
$$

if $M$ is odd and

$$
\beta_{i}=(i-1) \alpha
$$

if $M$ is even, for all values of $i$.

Proof. See the Appendix.

For Case $I^{*}$, we first want to do local calculations for a $I_{M_{1}}+I_{M_{2}}^{*}$ collision. We note that the values for $H_{C}^{2}\left(\bar{S}, i_{*} A\right)$ given below agree with those of [19] .

Theorem 3.5. Let $f: \bar{X} \rightarrow \bar{S}$ be a modified Miranda model with $\bar{S}$ strictly local, with $\Sigma=C \cup C^{\prime}, C$ and $C^{\prime}$ two curves meeting transversally, $C$ of fibre type $I_{M_{1}}$ and $C^{\prime}$ of fibre type $I_{M_{2}}^{*}$. Then

$$
H_{C}^{2}\left(\bar{S}, i_{*} A\right)= \begin{cases}\mathbf{Z} / 2 \mathbf{Z}, & M_{1} \text { odd } \\ \mathbf{Z} / 2 \mathbf{Z} \oplus \mathbf{Z} / 2 \mathbf{Z}, & M_{1}, M_{2} \text { even } \\ \mathbf{Z} / 4 \mathbf{Z}, & M_{1} \text { even, } M_{2} \text { odd } .\end{cases}
$$

(This gives more precise information than does Theorem 2.11 in this case.) Following the notation of Table 2 and identifying $H_{C}^{3}(\bar{S}, \mathcal{E})$ with $\operatorname{coker}\left(\mathbf{Q} / \mathbf{Z} \rightarrow(\mathbf{Q} / \mathbf{Z})^{m_{2}}\right)$ 
where $m_{2}$ is the number of components of $f^{-1}\left(C^{\prime}\right)$, the map $\phi_{1}: H_{C}^{2}\left(\bar{S}, i_{*} A\right) \rightarrow$ $H_{C}^{3}(\bar{S}, \mathcal{E})$ is given by representatives

$$
M_{1} \text { odd }, b \in \mathbf{Z} / 2 \mathbf{Z},\left(\phi_{1}(b)\right)_{i}= \begin{cases}0 & \text { on } Y_{i}^{\prime}, i \text { odd } \\ b & \text { on } Y_{i}^{\prime}, i \text { even } .\end{cases}
$$

$M_{1}, M_{2}$ even, $(b, c) \in \mathbf{Z} / 2 \mathbf{Z} \oplus \mathbf{Z} / 2 \mathbf{Z},\left(\phi_{1}(b, c)\right)_{i}= \begin{cases}0 & \text { on } Y_{i}^{\prime}, i \leq M_{2}+3, i \text { odd, } \\ b & \text { on } Y_{i}^{\prime}, i \leq M_{2}+2, i \text { even, } \\ c & \text { on } Y_{M_{2}+4}^{\prime} \\ b+c & \text { on } Y_{M_{2}+5}^{\prime} .\end{cases}$

In these two cases $b$ is the invariant along $C$.

$$
M_{1} \text { even, } M_{2} \text { odd }, b \in \mathbf{Z} / 4 \mathbf{Z},\left(\phi_{1}(b)\right)_{i}= \begin{cases}0 & \text { on } Y_{i}^{\prime}, i \leq M_{2}+2, i \text { odd }, \\ 2 b & \text { on } Y_{i}^{\prime}, i \leq M_{2}+3, i \text { even }, \\ b & \text { on } Y_{M_{2}+4}^{\prime}, \\ -b & \text { on } Y_{M_{2}+5}^{\prime} .\end{cases}
$$

Here $2 b$ is the invariant along $C$.

Proof. Removing the curve $C$ of fibre type $I_{M_{1}}$, putting $S=\bar{S}-C$ and $X=\bar{X} \times_{\bar{S}} S$, we use the spectral sequence of the proof of Prop. 1.5 to get an exact sequence

$$
0 \rightarrow H^{1}\left(S, P_{X / S}\right) \rightarrow \bigoplus_{i<j} H^{0}\left(Y_{i} \cap Y_{j}, \mathbf{Q} / \mathbf{Z}\right) \rightarrow \bigoplus_{i} H^{2}\left(Y_{i}, \mathbf{Q} / \mathbf{Z}\right) \rightarrow H^{2}\left(S, P_{X / S}\right) \rightarrow 0,
$$

where $Y_{1}, \ldots, Y_{m_{1}}$ are the divisors over $C$ in $\bar{X}$. Using Table 2 to calculate, one sees then in fact that $H^{1}\left(S, P_{X / S}\right)=0$, and we have an exact sequence

$$
0 \rightarrow \amalg_{S}(A) \rightarrow H^{2}(S, \mathcal{E}) \rightarrow H^{2}\left(S, P_{X / S}\right)
$$

The map $H^{2}(S, \mathcal{E}) \rightarrow H^{2}\left(S, P_{X / S}\right)$ factors through the natural specialization map $H^{2}(S, \mathcal{E})=H_{C}^{3}(\bar{S}, \mathcal{E}) \rightarrow \bigoplus_{i} H^{2}\left(Y_{i}, \mathbf{Q} / \mathbf{Z}\right)$, and thus $\amalg_{S}(A)$ can be calculated as the kernel of the map

$$
\bigoplus_{i<j} H^{0}\left(Y_{i} \cap Y_{j}, \mathbf{Q} / \mathbf{Z}\right) \oplus H^{2}(S, \mathcal{E}) \rightarrow \bigoplus_{i} H^{2}\left(Y_{i}, \mathbf{Q} / \mathbf{Z}\right)
$$

with the map $\amalg_{S}(A) \rightarrow H^{2}(S, \mathcal{E})$ corresponding to the map $\phi_{1}: H_{C}^{2}\left(\bar{S}, i_{*} A\right) \rightarrow$ $H_{C}^{3}(\bar{S}, \mathcal{E})$. Consider $b_{i} \in H^{0}\left(C_{i}, \mathbf{Q} / \mathbf{Z}\right)$ and $\left(a_{i}\right)_{1 \leq i \leq m_{2}}$ a representative of an element in $H^{2}(S, \mathcal{E})$, following the notation of Table 2. Proceeding as in the proof of Proposition 2.9 , we can identify $\bigoplus_{i} H^{2}\left(Y_{i}, \mathbf{Q} / \mathbf{Z}\right)$ with $\bigoplus_{i} H^{2}\left(f_{i}, \mathbf{Q} / \mathbf{Z}\right), f_{1}, \ldots, f_{m}$ the components of the central fibre, using the intersection pairing given in Table 2. Thus, in order to obtain zero in $\bigoplus_{i} H^{2}\left(Y_{i}, \mathbf{Q} / \mathbf{Z}\right)$, we must have the $b_{i}$ and $a_{i}$ 
satisfying the following equations:

$$
\begin{gathered}
f_{1}:-2 a_{1}+a_{3}=0 \\
f_{2}:-2 a_{2}+a_{3}=0 \\
f_{3}: a_{1}+a_{2}-2 a_{3}+a_{4}=0 \\
f_{4}: a_{3}-2 a_{4}+a_{5}=0 \\
\vdots \\
f_{n_{1}-1}: a_{n_{1}-2}-2 a_{n_{1}-1}+a_{n_{1}}=0 \\
f_{n_{1}}: b_{1}+a_{n_{1}-1}-a_{n_{1}}=0 \\
f_{n_{1}+1}:-b_{1}+b_{2}=0
\end{gathered}
$$

$\begin{array}{lll} & M_{1}, M_{2} \text { even } & M_{1} \text { even, } M_{2} \text { odd } \\ f_{M-4} & -b_{m_{1}-3}+b_{m_{1}-2}=0 & -b_{m_{1}-2}+b_{m_{1}-1}=0 \\ f_{M-3} & -b_{m_{1}-2}+b_{m_{1}-1}=0 & -b_{m_{1}-1}-a_{n_{1}}+a_{n_{1}+1}=0 \\ f_{M-2} & -b_{m_{1}-1}-a_{n_{1}}+a_{n_{1}+1}+a_{n_{1}+2}=0 & a_{n_{1}}-2 a_{n_{1}+1}+a_{n_{1}+2}+a_{n_{1}+3}=0 \\ f_{M-1} & a_{n_{1}}-2 a_{n_{1}+1}=0 & a_{n_{1}+1}-2 a_{n_{1}+2}=0 \\ f_{M} & a_{n_{1}}-2 a_{n_{1}+2}=0 & a_{n_{1}+1}-2 a_{n_{1}+3}=0 \\ & M_{1} \text { odd, } M_{2} \text { even } & M_{1}, M_{2} \text { odd } \\ f_{M-4} & -b_{m_{1}-3}+b_{m_{1}-2}=0 & -b_{m_{1}-2}+b_{m_{1}-1}=0 \\ f_{M-3} & -b_{m_{1}-2}+b_{m_{1}-1}=0 & -b_{m_{1}-1}-a_{n_{1}}+a_{n_{1}+1}=0 \\ f_{M-2} & -b_{m_{1}-1}-a_{n_{1}}+a_{n_{1}+1}=0 & a_{n_{1}}-2 a_{n_{1}+1}+a_{n_{1}+2}=0 \\ f_{M-1} & a_{n_{1}}-a_{n_{1}+1}+b_{m_{1}}=0 & b_{m_{1}}+a_{n_{1}+1}-a_{n_{1}+2}=0 \\ f_{M} & 2 b_{m_{1}}=0 & 2 b_{m_{1}}=0\end{array}$

Thus, as we can set $a_{1}=0$, we see that $a_{2}=0$ or $1 / 2$, and

$$
a_{i}= \begin{cases}0, & i \text { odd, } \\ a_{2}, & i \text { even },\end{cases}
$$

for $1 \leq i \leq n_{1}$, and that $b_{1}=b_{2}=\cdots=b_{m_{1}-1}=a_{2}$. For $M_{1}, M_{2}$ even, we see in addition that

$$
a_{n_{1}+1}, a_{n_{2}+2} \in\{0,1 / 2\}
$$

and

$$
a_{n_{1}+1}+a_{n_{1}+2}=a_{2} .
$$

Thus we get $\amalg_{S}(A)=\mathbf{Z} / 2 \mathbf{Z} \oplus \mathbf{Z} / 2 \mathbf{Z}$. In particular, the invariant along $C$ is $a_{2}$.

For $M_{1}$ even, $M_{2}$ odd, we have $a_{n_{1}+1}=a_{2}, a_{n_{1}+2}+a_{n_{1}+3}=0$, and $2 a_{n_{1}+2}=$ $2 a_{n_{1}+3}=a_{2}$, so we get $\amalg_{S}(A)=\mathbf{Z} / 4 \mathbf{Z}$. The invariant along $C$ is $a_{2}$.

For $M_{1}$ odd, $M_{2}$ even, we see that $a_{n_{1}+1}=a_{2}$, and we have $\amalg_{S}(A)=\mathbf{Z} / 2 \mathbf{Z}$.

For $M_{1}, M_{2}$ odd, we have $a_{n_{1}+1}=a_{2}, a_{n_{1}+2}=0$ and again, we get $\amalg_{S}(A)=$ $\mathbf{Z} / 2 \mathbf{Z}$.

This gives the desired results. If $M=1$, one makes a similar calculation and obtains the same results. 
Definition. If $\gamma \in H_{Z}^{2}\left(S, i_{*} A\right), s \in Z$ a collision point of type $I_{M_{1}}+I_{M_{2}}^{*}, M_{1}$ even, we say the local invariant of $\gamma$ at $s$ is $(b, c)$ if $M_{2}$ is even and the image of $\gamma$ in $\left(\underline{H}_{Z}^{2}\left(S, i_{*} A\right)\right)_{\bar{s}}=\mathbf{Z} / 2 \mathbf{Z} \oplus \mathbf{Z} / 2 \mathbf{Z}$ is $(b, c)$, as in Theorem 3.5; if $M_{2}$ is odd, we say the local invariant of $\gamma$ in $\left(\underline{H}_{Z}^{2}\left(S, i_{*} A\right)\right)_{\bar{s}}=\mathbf{Z} / 4 \mathbf{Z}$ is $b$.

Theorem 3.6. Let $C \subseteq Z$ be a curve of fibre type $I_{M}$ and case $I^{*}$, with generic point $t$, intersecting curves of fibre type $I_{M_{i}}, 1 \leq i \leq s$, and $I_{N_{i}}^{*}, 1 \leq i \leq u$. Let $\gamma \in H_{Z}^{2}\left(S, i_{*} A\right)$.

1) If $M$ is odd then $\phi_{t}(\gamma) \in H^{3}\left(S, \mathcal{E}_{t}\right)$ is zero.

2) If $M$ is even, the invariant of $\gamma$ along $C$ is $b$, and the local invariants of $\gamma$ at the collision $I_{M}+I_{N_{i}}^{*}$ are $\left(b, d_{i}\right)$ or $b_{i}$ with $b=2 b_{i}$ depending on whether $N_{i}$ is even or odd, then $\phi_{t}(\gamma) \in H^{3}\left(S, \mathcal{E}_{t}\right)=\operatorname{coker}\left(\mathbf{Q} / \mathbf{Z} \rightarrow(\mathbf{Q} / \mathbf{Z})^{M / 2+1}\right)$ is represented by

$$
\left(0, \ldots,-b a+\sum_{j=1}^{u} \alpha^{j}-\sum_{j=r+1}^{s} \frac{M c_{j}}{2}\right),
$$

with the components corresponding to $Y_{1}, \ldots, Y_{M / 2+1}$ respectively, $a$ is an integer which depends only on $X$ and $\alpha^{j}$ depends on the value of the local invariants at the collisions, with

$$
\alpha^{j}= \begin{cases}b+d_{j} & \text { if } N_{j} \text { is even }, \\ -b_{j} & \text { if } N_{j} \text { is odd } .\end{cases}
$$

Proof. See the Appendix.

Remark 3.7. The parity of the integer $a$ in the above theorem does not seem to be determinable from purely numeric data, but see the proof of Theorem 3.6 if you wish to compute it from the geometry of $X$. However, for many applications, it doesn't matter: see Example 3.8, (4).

Example 3.8. We continue the examples of 2.13.

1) Here $\mathcal{E}=0$ so $\operatorname{ker} \phi=H_{Z}^{2}\left(\mathbf{P}^{2}, i_{*} A\right)$.

2) We have $H_{Z_{1}}^{2}\left(\mathbf{P}^{2}, i_{*} A\right)=\mathbf{Z} / 2 \mathbf{Z} \oplus \mathbf{Z} / 2 \mathbf{Z}$. Let $\gamma \in H_{Z}^{2}\left(S, i_{*} A\right)$. Using Propositions 3.1 and 3.3 and the fact that $Z$ intersects $C$ an even number of times and that $\gamma$ is 2-torsion, it is easy to see that $\phi(\gamma)=0$. Thus $\operatorname{ker} \phi=H_{Z_{1}}^{2}\left(\mathbf{P}^{2}, i_{*} A\right)$.

For $Z_{2}$ we see from Proposition 3.3 that if we choose a non-zero section over $E_{2}$ which represents a non-zero element of the $\mathbf{Z} / 2 \mathbf{Z} \oplus \mathbf{Z} / 2 \mathbf{Z}$ part of $H_{Z_{2}}^{2}\left(S_{2}, i_{*} A\right)$, the contribution to at least one of the components over $C$ will be $1 / 2$ and never zero. Thus one sees that $\operatorname{ker} \phi=(\mathbf{Q} / \mathbf{Z})^{\oplus 2}=H_{l}^{2}\left(S_{2}, i_{*} A\right)$, as $l$ does not intersect the discriminant locus.

3) We check the contributions for $\phi$ over the various components of $\Sigma$. Let $\gamma \in H_{Z}^{2}\left(S, i_{*} A\right) . \gamma$ has, in particular, invariant 0 along the curves of type $I_{2}$ and invariant 0 or $1 / 2$ along the curve of type $I_{1}$. If $t$ is the generic point of a curve of type $I_{2}$, then $\phi_{t}(\gamma)=0$ by Theorem 3.4, and if $t$ is the generic point of the curve of type $I_{1}$, then $\phi_{t}(\gamma)=0$ by Theorem 3.6. Over a curve $C$ of type $I_{0}^{*}$, one can check that $f_{3}^{-1}(C)$ consists of two reduced components and one multiple component. One of the reduced components intersects $\sigma_{0}$ and the other factors through a curve mapping 3-1 to $C$ (because of the collision $I I+I_{0}^{*}[16]$ ). Thus adding up all the local contributions from Theorem 3.5 and $(3.1)$, we obtain $\phi_{t}(\gamma)=0$ in $H^{3}\left(S, \mathcal{E}_{t}\right)$ where $t$ is the generic point of $C$. 
Finally we have a curve $D$ of fibre type $I I I$, with $f^{-1}(D)$ consisting of two components $Y_{1}$ and $Y_{2}$. By (3.1) $Y_{1}$ gets a contribution of 0 and $Y_{2} 0$ or $1 / 2$ depending on whether we want an isolated multiple fibre at the $I_{0}^{*}+I I I$ collisions. This shows that it is not possible to have an isolated multiple fibre there, and so $\operatorname{ker} \phi=\mathbf{Z} / 2 \mathbf{Z}$, the only possibility being to have multiplicity two fibres over the $I_{1}$ curve.

4) Here over each curve of type $I_{0}^{*}$ there are two reduced components, one factoring through a 3-1 cover of the curve, again because of the $I I+I_{0}^{*}$ collisions. Theorems 3.5 and 3.6 then tell us $\operatorname{ker} \phi=\mathbf{Z} / 2 \mathbf{Z} \oplus \mathbf{Z} / 2 \mathbf{Z}$, the possibilities being to have isolated multiple fibres at $P_{1}$ and $P_{2}$ at the same time, or else to have fibre-type ${ }_{2} I_{2}$ along $Z$. Notice that we haven't computed the integer $a$ of Theorem 3.6 ; this would tell us precisely which subgroup of $H_{Z}^{2}\left(S, i_{*} A\right)$ the kernel of $\phi$ is. However, this information is not particularly helpful.

\section{THE SECOND OBSTRUCTION}

We will now consider the nature of the second obstruction. Recall from $\S 3$ that we have maps

$$
H_{Z}^{2}\left(S, i_{*} A\right) \stackrel{\phi}{\longrightarrow} H^{3}(S, \mathcal{E})
$$

and

$$
\operatorname{ker} \phi \stackrel{\psi}{\longrightarrow} G(X / S):=\operatorname{coker}\left(H^{2}(S, \mathcal{E}) \rightarrow H^{2}\left(S, P_{X / S}\right)\right)
$$

with

$$
\text { ker } \psi \cong \amalg_{S-Z}(A) / \amalg_{S}(A) .
$$

To understand the map $\psi$, we need

Definition. Let $\left\{Y_{i}^{j}\right\}$ be the set of prime divisors of $X$ mapping to curves $Z_{i}$ on $S$ contained in the discriminant locus, and let $i: \tilde{Y}_{i}^{j} \rightarrow X$ be the map of the normalization of $Y_{i}^{j}$ to $X$, and $\tilde{Y}_{i}^{j} \stackrel{\pi}{\longrightarrow} \bar{Z}_{i}^{j} \longrightarrow Z_{i}$ be the Stein factorization of the map $\tilde{Y}_{i}^{j} \longrightarrow Z_{i}$. We put

$$
C(X / S)=\frac{C H^{2}(X)_{\text {tors }}}{f^{*} C H^{2}(S)_{\text {tors }}+\sum_{i, j} i_{*} \pi^{*} \operatorname{Pic}\left(\bar{Z}_{i}^{j}\right)_{\text {tors }}} .
$$

Theorem 4.1. Suppose $S$ (and hence $X$ ) is projective. Then there is an injective map $\lambda: C(X / S) \rightarrow G(X / S)$ through which the map $\psi$ factors, giving a map $\psi^{\prime}$ : $\operatorname{ker} \phi \rightarrow C(X / S)$ with $\operatorname{ker} \psi^{\prime}=\operatorname{ker} \psi$.

To prove this, we will need

Theorem 4.2. Let $X$ be a projective variety over an algebraically closed field $k$ of characteristic zero, $Z \subseteq X$ a closed subset, $l$ a prime number. Then there is a factorization of the natural map

$$
H_{Z}^{3}\left(X, \mathbf{Q}_{l} / \mathbf{Z}_{l}(2)\right) \rightarrow H^{3}\left(X, \mathbf{Q}_{l} / \mathbf{Z}_{l}(2)\right)
$$

via

$$
H_{Z}^{3}\left(X, \mathbf{Q}_{l} / \mathbf{Z}_{l}(2)\right) \longrightarrow C H^{2}(X)(l) \stackrel{\lambda_{2}^{l}}{\longrightarrow} H^{3}\left(X, \mathbf{Q}_{l} / \mathbf{Z}_{l}(2)\right)
$$

where $C H^{2}(X)(l)$ is the l-primary part of the second Chow group of $X$, and $\lambda_{2}^{l}$ is the Bloch cycle map. (See [2] and later in this section for the construction and properties of the Bloch map.) Furthermore, $\lambda_{2}^{l}$ is injective. Over an algebraically 
closed field, as usual we can choose an identification of $\mu_{n}$ with $\mathbf{Z} / n \mathbf{Z}$ for each $n$, and this yields by (1.4) a factorization

$$
H_{Z}^{3}\left(X, \mathbf{G}_{m}\right) \rightarrow C H^{2}(X)_{t o r s} \stackrel{\lambda_{X}}{\longrightarrow} H^{3}\left(X, \mathbf{G}_{m}\right)
$$

with $\lambda_{X}$ injective.

This theorem will be proved in the discussion of the Bloch map later in this section.

Proof of Theorem 4.1. Since $H^{2}\left(S, P_{X / S}\right)=H^{3}\left(X, \mathbf{G}_{m}\right) / H^{3}\left(S, \mathbf{G}_{m}\right)$, by Theorem 4.2 we get a natural map $C H^{2}(X)_{\text {tors }} / f^{*} C H^{2}(S)_{\text {tors }} \stackrel{\lambda^{\prime}}{\longrightarrow} H^{2}\left(S, P_{X / S}\right)$. This map is in fact an injection. Indeed, suppose $\alpha \in C H^{2}(X)_{\text {tors }}$ is such that $\lambda_{X}(\alpha)=f^{*}(\beta)$ for some $\beta \in H^{3}\left(S, \mathbf{G}_{m}\right)$. Recall we have a section $\sigma_{0}: S \rightarrow X$ inducing maps $\sigma_{0}^{*}: C H^{2}(X)_{t o r s} \rightarrow C H^{2}(S)_{\text {tors }}$ and $\sigma_{0}^{*}: H^{3}\left(X, \mathbf{G}_{m}\right) \rightarrow H^{3}\left(S, \mathbf{G}_{m}\right)$, which are left inverses to $f^{*}$. So

$$
\begin{aligned}
\lambda_{X}(\alpha) & =f^{*} \sigma_{0}^{*} \lambda_{X}(\alpha) \\
& =f^{*} \lambda_{S} \sigma_{0}^{*}(\alpha) \\
& =\lambda_{X} f^{*} \sigma_{0}^{*}(\alpha) .
\end{aligned}
$$

Thus

$$
\begin{aligned}
\lambda_{X}\left(\alpha-f^{*} \sigma_{0}^{*}(\alpha)\right) & =\lambda_{X}(\alpha)-\lambda_{X}(\alpha) \\
& =0
\end{aligned}
$$

Since $\lambda_{X}$ is injective, $\alpha=f^{*} \sigma_{0}^{*}(\alpha)$, and so the class of $\alpha$ in

$$
C H^{2}(X)_{t o r s} / f^{*} C H^{2}(S)_{t o r s}
$$

is zero. We conclude that $\lambda^{\prime}$ is injective.

The existence and injectivity of $\lambda$ follows from the commutative diagram

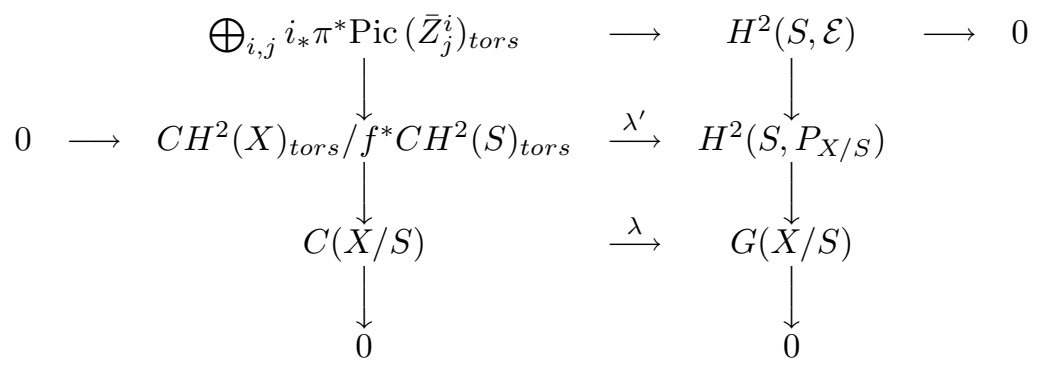

To show that $\psi$ factors through $\lambda$, we can always enlarge $Z$, for if $Z \subseteq Z^{\prime}$, we have $H_{Z}^{2}\left(S, i_{*} A\right) \subseteq H_{Z^{\prime}}^{2}\left(S, i_{*} A\right)$, so we can assume that $\Sigma \subseteq Z$. Then ker $\phi=$ 
$\operatorname{ker}\left(H_{Z}^{2}\left(S, i_{*} A\right) \rightarrow H_{Z}^{3}(S, \mathcal{E})\right)$ since $H_{Z}^{3}(S, \mathcal{E})=H^{3}(S, \mathcal{E})$. Thus we have a diagram

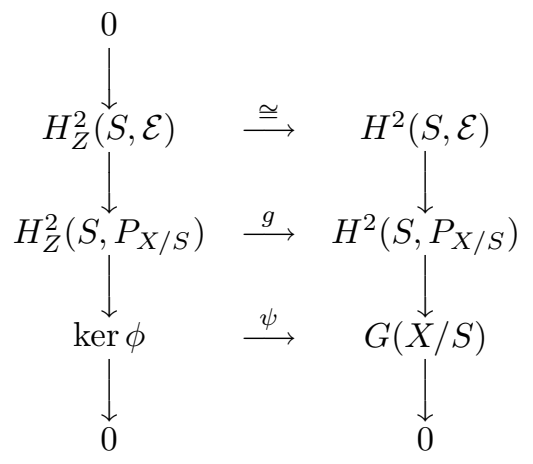

By Theorem 4.2, $g$ factors through $\frac{C H^{2}(X)_{\text {tors }}}{f^{*} C H^{2}(S)_{\text {tors }}} \subseteq H^{2}\left(S, P_{X / S}\right)$, and so $\psi$ factors as desired.

This is not so helpful unless we understand the nature of the factorization of Theorem 4.2. Our goal then for the remainder of this paper is to understand this factorization, and to use this understanding to sketch how to explicitly construct elements of $C(X / S)$ from elements of ker $\phi$. We will then give some examples and some important special cases.

We begin by reviewing Bloch-Ogus theory. Let $X$ be a quasi-projective variety of finite type over an algebraically closed field $k$ of characteristic 0 . Denote by $\underline{H}^{i}\left(\mu_{n}^{\otimes r}\right)$ the Zariski sheaf on $X$ associated to the presheaf

$$
U \mapsto H^{i}\left(U, \mu_{n}^{\otimes r}\right) ;
$$

i.e. $\underline{H}^{i}\left(\mu_{n}^{\otimes r}\right)=R^{i} j_{*} \mu_{n}^{\otimes r}$ where $j$ is the morphism from the étale to the Zariski site on $X$. By the Leray spectral sequence, we have a spectral sequence

$$
E_{2}^{p, q}=H_{Z a r}^{p}\left(X, \underline{H}^{q}\left(\mu_{n}^{\otimes r}\right)\right) \Rightarrow H^{p+q}\left(X, \mu_{n}^{\otimes r}\right) .
$$

We have the following resolution of the sheaf $\underline{H}^{q}\left(\mu_{n}^{\otimes r}\right)$ by flasque sheaves in the Zariski topology, given in [3] :

$$
\begin{aligned}
0 \rightarrow \underline{H}^{q}\left(X, \mu_{n}^{\otimes r}\right) & \rightarrow \bigoplus_{x \in X^{(0)}} i_{x} H^{q}\left(x, \mu_{n}^{\otimes r}\right) \rightarrow \bigoplus_{x \in X^{(1)}} i_{x} H^{q-1}\left(x, \mu_{n}^{\otimes(r-1)}\right) \rightarrow \cdots \\
& \rightarrow \bigoplus_{x \in X^{(q)}} i_{x} H^{0}\left(x, \mu_{n}^{\otimes(r-q)}\right) \rightarrow 0
\end{aligned}
$$

where

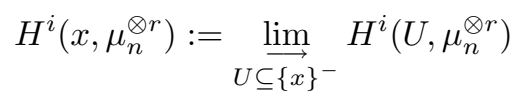

and $i_{x} G$ is the constant sheaf $G$ on $\{x\}^{-}$extended by zero outside of this set, $G$ an abelian group.

One can compute Zariski cohomology using the resolution (4.1), and in particular,

$$
H_{Z a r}^{p}\left(X, \underline{H}^{q}\left(\mu_{n}^{\otimes r}\right)\right)=0 \quad \forall p>q .
$$

The above spectral sequence tells us that we have an exact sequence

$$
0 \rightarrow H_{Z a r}^{1}\left(X, \underline{H}^{2}\left(\mu_{n}^{\otimes r}\right)\right) \rightarrow H^{3}\left(X, \mu_{n}^{\otimes r}\right) \rightarrow H^{0}\left(X, \underline{H}^{3}\left(\mu_{n}^{\otimes r}\right)\right),
$$


the last group being contained in $H^{3}\left(K(X), \mu_{n}^{\otimes r}\right)$ by the resolution (4.1) for $q=3$. Thus $H_{Z a r}^{1}\left(X, \underline{H}^{2}\left(\mu_{n}^{\otimes r}\right)\right)$ is the set of classes in $H^{3}\left(X, \mu_{n}^{\otimes r}\right)$ with support on a closed subset of $X$. In particular, the image of $H_{Z}^{3}\left(X, \mu_{n}^{\otimes r}\right)$ in $H^{3}\left(X, \mu_{n}^{\otimes r}\right)$ is contained in $H_{Z a r}^{1}\left(X, \underline{H}^{2}\left(\mu_{n}^{\otimes r}\right)\right)$. In fact, using the spectral sequence

$$
E_{2}^{p, q}=H_{Z}^{p}\left(X, \underline{H}^{q}\left(\mu_{n}^{\otimes r}\right)\right) \Rightarrow H_{Z}^{p+q}\left(X, \mu_{n}^{\otimes r}\right)
$$

and the Bloch-Ogus resolution (4.1) which shows $H_{Z}^{0}\left(X, \underline{H}^{3}\left(\mu_{n}^{\otimes 2}\right)\right)=0$, we see that

$$
H_{Z}^{3}\left(X, \mu_{n}^{\otimes 2}\right)=H_{Z}^{1}\left(X, \underline{H}^{2}\left(\mu_{n}^{\otimes 2}\right)\right),
$$

where the latter local cohomology group is in the Zariski topology. Recalling that $Z$ is a divisor, this is then the cohomology of the complex

$$
H_{Z}^{0}\left(X, i_{x} H^{2}\left(K(X), \mu_{n}^{\otimes 2}\right)\right)=0 \rightarrow \bigoplus_{x \in Z^{(0)}} k(x)^{*} / k(x)^{* n} \stackrel{\partial_{n}^{Z}}{\longrightarrow} \bigoplus_{x \in Z^{(1)}} \mathbf{Z} / n \mathbf{Z}
$$

$(k(x)$ is the residue field of the point $x \in X)$ where $\partial_{n}^{Z}$ is reduction modulo $n$ of the natural map $\partial^{Z}$ in

$$
\bigoplus_{x \in Z^{(0)}} k(x)^{*} \stackrel{\partial^{Z}}{\longrightarrow} \bigoplus_{x \in Z^{(1)}} \mathbf{Z} \longrightarrow C H^{1}(Z) \longrightarrow 0
$$

where we take this exact sequence as the suitable definition for $C H^{1}(Z)$ if $Z$ is singular (i.e. the Fulton Chow groups [10] ). Thus

$$
H_{Z}^{3}\left(X, \mu_{n}^{\otimes 2}\right)=\operatorname{ker} \partial_{n}^{Z} .
$$

We also have similar sequences for $X: H_{Z a r}^{1}\left(\underline{H}^{2}\left(X, \mu_{n}^{\otimes 2}\right)\right)$ is the cohomology of the complex

$$
H^{2}\left(K(X), \mu_{n}^{\otimes 2}\right) \longrightarrow \bigoplus_{x \in X^{(1)}} k(x)^{*} / k(x)^{* n} \stackrel{\partial_{n}^{X}}{\longrightarrow} \bigoplus_{x \in X^{(2)}} \mathbf{Z} / n \mathbf{Z}
$$

where $\partial_{n}^{X}$ is reduction modulo $n$ of the natural map $\partial^{X}$ in

$$
\bigoplus_{x \in X^{(1)}} k(x)^{*} \stackrel{\partial^{X}}{\longrightarrow} \bigoplus_{x \in X^{(2)}} \mathbf{Z} \rightarrow C H^{2}(X) \rightarrow 0 .
$$

Finally, the snake lemma for the diagram

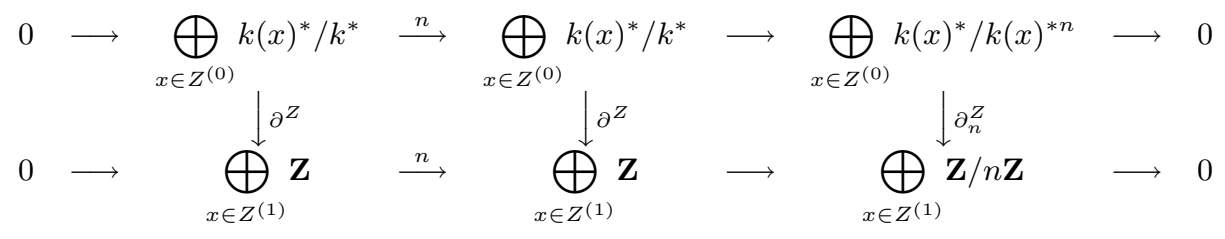

gives us an exact sequence

$$
0 \rightarrow \operatorname{ker} \partial^{Z} / n \operatorname{ker} \partial^{Z} \rightarrow H_{Z}^{3}\left(X, \mu_{n}^{\otimes 2}\right) \rightarrow{ }_{n} C H^{1}(Z) \rightarrow 0 .
$$


The similar diagram for $X[2,(2.1)]$ gives

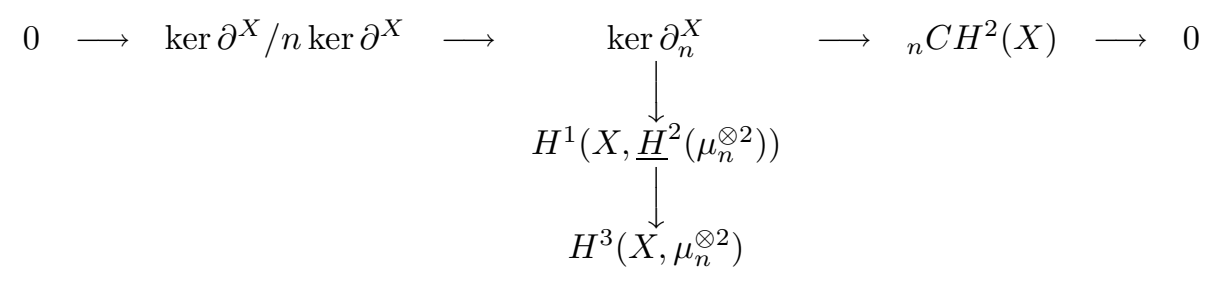

(only the row is exact) and the map $H_{Z}^{3}\left(X, \mu_{n}^{\otimes 2}\right) \rightarrow H^{3}\left(X, \mu_{n}^{\otimes 2}\right)$ is clearly compatible with the map $\operatorname{ker} \partial_{n}^{Z} \rightarrow \operatorname{ker} \partial_{n}^{X}$.

The map $\operatorname{ker} \partial_{n}^{X} \rightarrow{ }_{n} C H^{2}(X)$ has the following explicit description: given $\left(\bar{f}_{x}\right)_{x \in X^{(1)}} \in \operatorname{ker} \partial_{n}^{X}$ with $f_{x} \in k(x)^{*}$ representatives for $\bar{f}_{x}$, we obtain the associated principal algebraic 2-cycle

$$
0 \sim \sum n_{i} C_{i}=\sum_{x \in X^{(1)}}\left(f_{x}\right)
$$

and we have $n \mid n_{i} \forall i$. Thus $\sum \frac{n_{i}}{n} C_{i}$ is an $n$-torsion cycle, which is well-defined up to rational equivalence.

Bloch proves, using the Weil conjectures and assuming $X$ is projective, that if one considers the induced map

$$
\rho: \operatorname{ker} \partial^{X} \rightarrow \lim _{l^{\nu}} H^{3}\left(X, \mu_{l^{\nu}}^{\otimes 2}\right)=H^{3}\left(X, \mathbf{Z}_{l}(2)\right)
$$

the image of $\rho$ is torsion, and hence the map obtained by taking the direct limit over $l^{\nu}$

$$
\operatorname{ker} \partial^{X} \otimes \mathbf{Q}_{l} / \mathbf{Z}_{l} \rightarrow H^{3}\left(X, \mathbf{Q}_{l} / \mathbf{Z}_{l}(2)\right)
$$

is zero. The induced map $\lambda_{2}^{l}: C H^{2}(X)(l) \rightarrow H^{3}\left(X, \mathbf{Q}_{l} / \mathbf{Z}_{l}(2)\right)$ is, up to sign, the Bloch map. Thus we see, in particular, that if $X$ is projective then the map

$$
H_{Z}^{3}\left(X, \mathbf{Q}_{l} / \mathbf{Z}_{l}(2)\right) \rightarrow H^{3}\left(X, \mathbf{Q}_{l} / \mathbf{Z}_{l}(2)\right)
$$

factors as

$$
H_{Z}^{3}\left(X, \mathbf{Q}_{l} / \mathbf{Z}_{l}(2)\right) \rightarrow C H^{1}(Z)(l) \longrightarrow C H^{2}(X)(l) \stackrel{\lambda_{2}^{l}}{\longrightarrow} H^{3}\left(X, \mathbf{Q}_{l} / \mathbf{Z}_{l}(2)\right) .
$$

Furthermore, by [5] Cor. 4, the Bloch map is injective for codimension 2 cycles. Taking the limit over $l$, working over an algebraically closed field of characteristic zero and choosing identifications of $\mu_{n}$ with $\mathbf{Z} / n \mathbf{Z}$, one obtains

$$
H_{Z}^{3}(X, \mathbf{Q} / \mathbf{Z}) \rightarrow C H^{1}(Z)_{\text {tors }} \rightarrow C H^{2}(X)_{\text {tors }} \rightarrow H^{3}(X, \mathbf{Q} / \mathbf{Z})
$$

which by (1.4) gives the desired factorization

$$
H_{Z}^{3}\left(X, \mathbf{G}_{m}\right) \rightarrow C H^{1}(Z)_{\text {tors }} \rightarrow C H^{2}(X)_{\text {tors }} \rightarrow H^{3}\left(X, \mathbf{G}_{m}\right) .
$$

This proves Theorem 4.2 .

We now make several other comments which will be useful in understanding these maps. 
Note that we have the diagram, for $U=X-Z$,

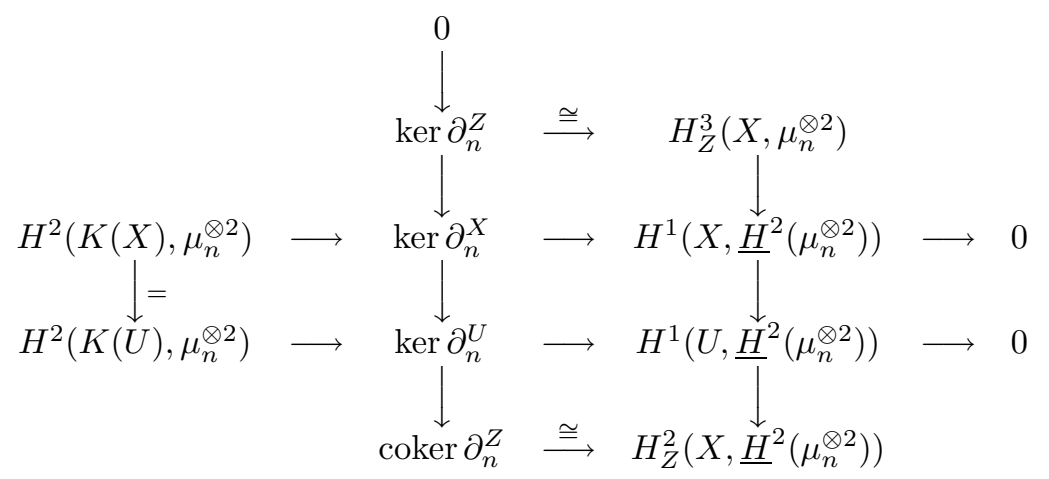

A similar argument where $C$ is a projective non-singular curve and $Z \subseteq C$ a closed subset provides a diagram

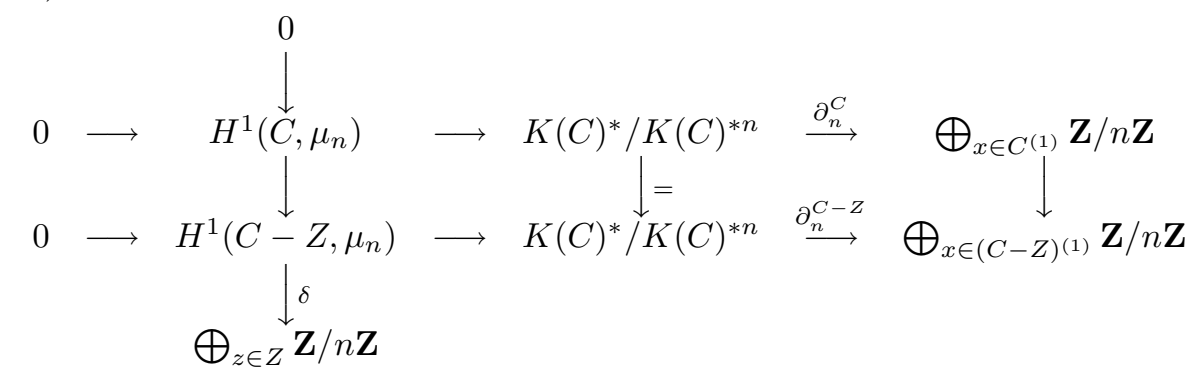

where the map $\delta$ is the projection of $\partial_{n}^{C}$ restricted to $H^{1}\left(C-Z, \mu_{n}\right)$ onto the factor $\bigoplus_{z \in Z} \mathbf{Z} / n \mathbf{Z}$.

We now return to understanding the map $\psi^{\prime}$ in Theorem 4.1. How do we calculate the map $\psi^{\prime}$ to find our final obstruction? We will sketch the method in general here and give a number of examples. In the proof of Theorem 4.1, $Z$ was enlarged in such a way that $f^{-1}(Z)$ might not be s.n.c., which makes calculations harder, so it is easiest to actually split ker $\phi$ up into two parts. Thus we work with the assumption that $f^{-1}(Z)$ is s.n.c., which holds if $Z$ is s.n.c. and the only components of $\Sigma$ it contains are of fibre type $I_{M}$. Let

$$
G_{1}=\operatorname{ker}\left(H_{Z}^{2}\left(S, i_{*} A\right) \rightarrow H_{Z}^{3}(S, \mathcal{E})\right)
$$

from this one obtains an exact sequence

$$
0 \rightarrow G_{1} \rightarrow \operatorname{ker} \phi \rightarrow G_{2} \rightarrow 0
$$


fitting into a diagram

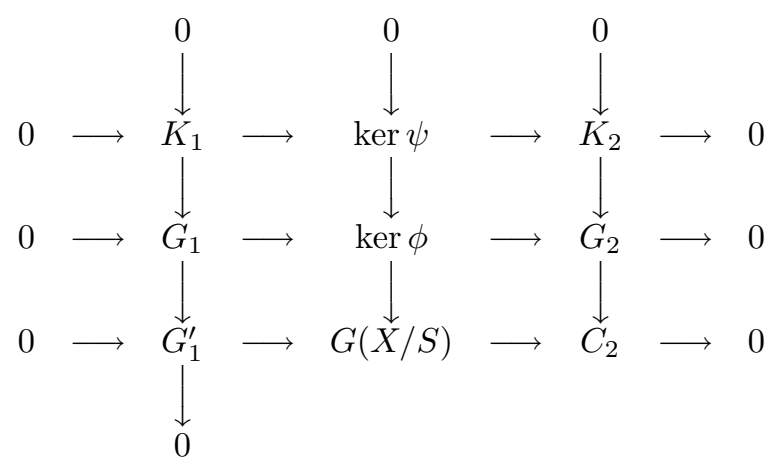

with $K_{1}=G_{1} \cap \operatorname{ker} \psi$. We calculate $K_{1}$ and $K_{2}$.

As far as the map $G_{1} \rightarrow G(X / S)$ is concerned, we have a commutative diagram

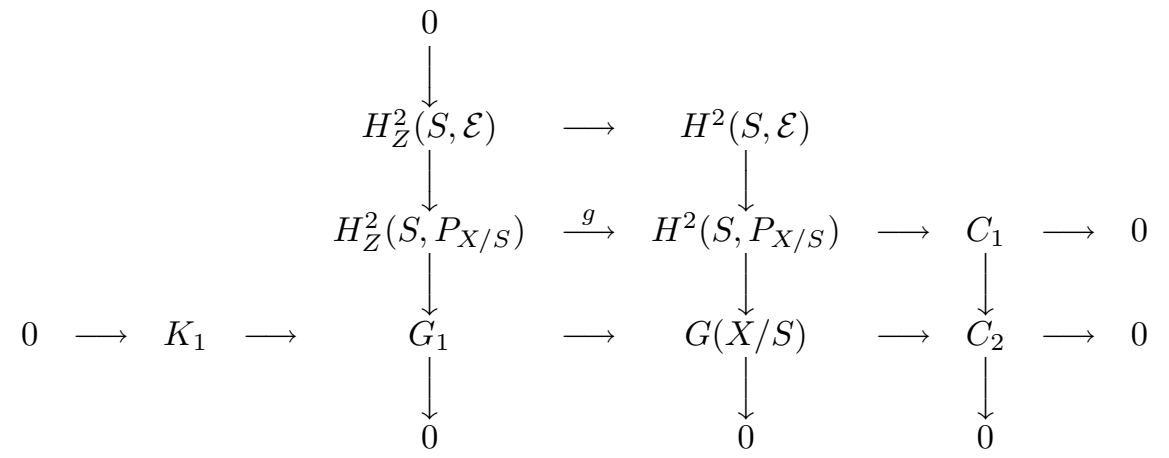

where $g$ is interpreted by factoring through the Bloch map, as before.

For $G_{2}$ we have a diagram

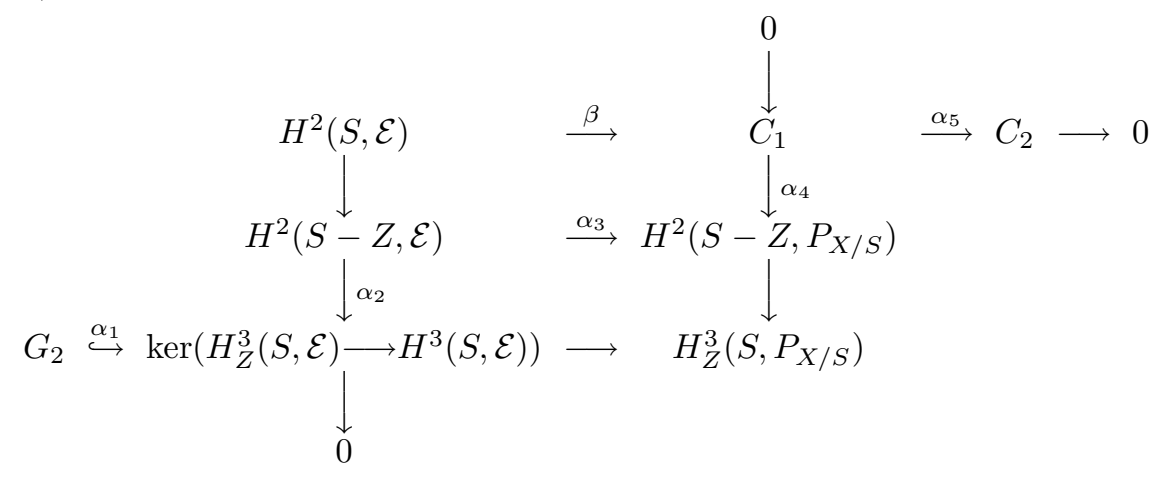

The kernel of the map $G_{2} \rightarrow C_{2}$ given by the snake lemma is then $K_{2}$.

To interpret these maps, we use our discussion of Bloch-Ogus theory. By Theorem 1.1 and $(4.6), H^{2}(S, \mathcal{E}), H^{2}(S-Z, \mathcal{E})$ and $\operatorname{ker}\left(H_{Z}^{3}(S, \mathcal{E}) \rightarrow H^{3}(S, \mathcal{E})\right)$ are subquotients of

$$
\bigoplus_{t \in \Sigma^{(0)}} \bigoplus_{i} \operatorname{ker}\left(K\left(C\left(t_{i}\right)\right)^{*} \otimes \mathbf{Q} / \mathbf{Z} \rightarrow \bigoplus_{x \in C\left(t_{i}\right)} \mathbf{Q} / \mathbf{Z}\right)
$$




$$
\bigoplus_{t \in(\Sigma-Z)^{(0)}} \bigoplus_{i} \operatorname{ker}\left(K\left(C\left(t_{i}\right)\right)^{*} \otimes \mathbf{Q} / \mathbf{Z} \rightarrow \bigoplus_{x \in C\left(t_{i}\right)-q_{t}^{-1}(Z)} \mathbf{Q} / \mathbf{Z}\right)
$$

and

$$
\bigoplus_{t \in(\Sigma-Z)^{(0)}} \bigoplus_{x \in q_{t}^{-1}(Z \cap C(t))} \mathbf{Q} / \mathbf{Z}
$$

respectively, while by (4.5) and (4.9), the images of $\beta$ and $\alpha_{3}$ are subquotients of $\lim _{n} \operatorname{ker} \partial_{n}^{X}$ and $\varliminf_{n} \operatorname{ker} \partial_{n}^{U}$ respectively. These groups fit into the following diagram, compatible with (4.10):

$$
\begin{aligned}
& \bigoplus_{t \in \Sigma^{(0)}} \bigoplus_{i} \operatorname{ker}\left(K\left(C\left(t_{i}\right)\right)^{*} \otimes \mathbf{Q} / \mathbf{Z} \rightarrow \bigoplus_{x \in C\left(t_{i}\right)} \mathbf{Q} / \mathbf{Z}\right) \quad \longrightarrow \underset{n}{\longrightarrow} \lim _{\longrightarrow} \operatorname{ker} \partial_{n}^{X} \\
& \bigoplus_{t \in(\Sigma-Z)^{(0)}} \bigoplus_{i} \operatorname{ker}\left(K\left(C\left(t_{i}\right)\right)^{*} \stackrel{\downarrow}{\otimes} \mathbf{Q} / \mathbf{Z} \rightarrow \bigoplus_{x \in C\left(t_{i}\right)-q_{t}^{-1}(Z)} \mathbf{Q} / \mathbf{Z}\right) \stackrel{\alpha_{3}^{\prime}}{\longrightarrow} \underset{n}{\lim } \underset{n}{\underset{n}{\mid} \operatorname{ker}} \partial_{n}^{U} \\
& \bigoplus_{t \in(\Sigma-Z)^{(0)}} \bigoplus_{x \in q_{t}^{-1}(Z \cap C(t))} \downarrow_{\alpha_{2}^{\prime}} \mathbf{Q} / \mathbf{Z}
\end{aligned}
$$

The map $\alpha_{1}$ can be understood using $\S 3$. If $\gamma \in G_{2}$, we choose some suitable representative $\gamma_{1}$ of $\alpha_{1}(\gamma)$ in $\bigoplus_{t \in(\Sigma-Z)^{(0)}} \bigoplus_{x \in q_{t}^{-1}(Z \cap C(t))} \mathbf{Q} / \mathbf{Z} . \gamma_{1}$ can be pulled back via $\alpha_{2}^{\prime}$, using the precise interpretation of the map in (4.6) corresponding to $\delta$, and then pushed forward to $\gamma_{3}$ via $\alpha_{3}^{\prime}$, considering the rational functions on $C\left(t_{i}\right)$ as rational functions on the corresponding divisors in $X . \gamma_{3}$ can in turn be pulled back via $\alpha_{4}^{\prime}$ to $\gamma_{4}$ by finding suitable rational functions on the components of $f^{-1}(Z)$, using the explicit maps of (4.5). Finally, via (4.3), this gives a 2-cycle on $X$, and the class of this cycle in $C(X / S)$ is the desired element.

We now give a representative special case of this construction which is quite important. If the reader should need to compute the obstruction in a more complicated case when $C(X / S) \neq 0$, then this case along with those of the examples should provide a sufficient guide.

Theorem 4.3. Given the hypothesis of Theorem 4.1, assume furthermore that $Z$ is an irreducible non-singular curve disjoint from $\Sigma$. Let $\gamma \in H_{Z}^{2}\left(S, i_{*} A\right)$ be represented by $\sigma \in M W\left(f^{-1}(Z) / Z\right)$, with $\sigma_{0}$ the zero section. If $\iota: f^{-1}(Z) \rightarrow X$ is the inclusion, then $\phi(\gamma)=0$ and $\psi^{\prime}(\gamma)$ is the class of $\iota_{*}\left[\sigma-\sigma_{0}\right]$ in $C(X / S)$.

Proof. In fact, since $Z \cap \Sigma=\phi, H_{Z}^{2}(S, \mathcal{E})=H_{Z}^{3}(S, \mathcal{E})=0$ and $G_{1}=\operatorname{ker} \phi=$ $H_{Z}^{2}\left(S, i_{*} A\right)=H_{Z}^{2}\left(S, P_{X / S}\right)$, and we have the map of (4.9)

$$
G_{1}=H_{Z}^{2}\left(S, P_{X / S}\right) \stackrel{g}{\longrightarrow} H^{2}\left(S, P_{X / S}\right) .
$$

Now

$$
\begin{aligned}
H_{Z}^{2}\left(S, P_{X / S}\right) & =H_{Z}^{3}\left(X, \mathbf{G}_{m}\right) / H_{Z}^{3}\left(S, \mathbf{G}_{m}\right) \\
& =H^{1}\left(f^{-1}(Z), \mathbf{Q} / \mathbf{Z}\right) / H^{1}(Z, \mathbf{Q} / \mathbf{Z}) \\
& =C H^{1}\left(f^{-1}(Z)\right)_{\text {tors }} / f^{*} C H^{1}(Z)_{\text {tors }},
\end{aligned}
$$

and $g$ is induced via the factorization

$$
C H^{1}\left(f^{-1}(Z)\right)_{\text {tors }} \stackrel{\iota_{*}}{\longrightarrow} C H^{2}(X)_{\text {tors }} \stackrel{\lambda_{X}}{\longrightarrow} H^{3}\left(X, \mathbf{G}_{m}\right) .
$$

The class in $C H^{1}\left(f^{-1}(Z)\right)_{\text {tors }}$ corresponding to $\sigma$ is the torsion cycle $\left[\sigma-\sigma_{0}\right]$, and thus $\psi^{\prime}(\gamma)=\iota_{*}\left[\sigma-\sigma_{0}\right] \in C(X / S)$. 
Remark 4.4. More generally, suppose $Z$ is an irreducible curve which intersects $\Sigma$ transversally, and an element $\gamma \in H_{Z}^{2}\left(S, i_{*} A\right)$ is given by $\sigma \in M W\left(f^{-1}(Z) / Z\right)$, with $\phi(\gamma)=0$. Then using (4.10) and (4.11), one finds that $\psi^{\prime}(\gamma)=\iota_{*}\left[\sigma-\sigma_{0}\right]+\alpha$, where $\alpha$ is a cycle supported on $f^{-1}(\Sigma)$. See the second example below for a specific calculation.

Example 4.5. We continue the examples of 2.13 and 3.8.

1) Here $C H^{2}\left(X_{1}\right)=\mathbf{Z} p_{1}^{*} P \oplus\left(p_{2}^{*} \operatorname{Pic} E\right) \cdot p_{1}^{*} l$ where $p_{1}, p_{2}: \mathbf{P}^{2} \times E \rightarrow \mathbf{P}^{2}, E$ are the first and second projections, $P \in \mathbf{P}^{2}$ the class of a point and $l \subseteq \mathbf{P}^{2}$ the class of a line. Thus $C\left(X_{1} / \mathbf{P}^{2}\right)=C H^{2}\left(X_{1}\right)_{t o r s}=(\operatorname{Pic} E)_{\text {tors }}$. Thus by Theorem 4.3, $\psi^{\prime}$ takes $\sigma_{i}-\sigma_{0} \in\left(\operatorname{Pic}\left(Z_{i} \times E\right)\right)_{\text {tors }}$, where $\sigma_{i}$ is a torsion section of $p_{1}^{-1}\left(Z_{i}\right)$ with $\sigma_{0}$ the zero section, to the 2-cycle $\sigma_{i}-\sigma_{0}$, which can be written as $p_{1}^{*} Z_{i} \cdot p_{2}^{*}\left(P_{i}-P_{0}\right)$, $P_{i}, P_{0} \in E$. This is rationally equivalent to

$$
\left(\operatorname{deg} Z_{i}\right) p_{1}^{*} l \cdot p_{2}^{*}\left(P_{i}-P_{0}\right)
$$

Thus, if $\gamma \in H_{Z}^{2}\left(S, i_{*} A\right)=H_{Z}^{2}\left(S, P_{X / S}\right)$ is determined by points $P_{i} \in E, 1 \leq i \leq n$, $\left(Z=Z_{1} \cup \cdots \cup Z_{n}\right)$ with $P_{i}-P_{0} \in(\operatorname{Pic} E)_{\text {tors }}$, then we have

$$
\psi^{\prime}(\gamma)=\sum_{i=1}^{n} \operatorname{deg} Z_{i}\left(P_{i}-P_{0}\right) \in C\left(X_{1} / \mathbf{P}^{2}\right)=(\text { Pic } E)_{\text {tors }} .
$$

Thus we have the following special cases:

$n=1: Z$ is an irreducible non-singular curve of degree $d$. Then

$$
\amalg_{\mathbf{P}^{2}-Z}(A) / \amalg_{\mathbf{P}^{2}}(A)={ }_{d} \operatorname{Pic} E=(\mathbf{Z} / d \mathbf{Z})^{\oplus 2} .
$$

Thus the multiplicity along $Z$ must divide $d$. (See [9] for the first proof of this fact.)

$n=2$ : Let $Z$ be the union of two lines in $\mathbf{P}^{2}$. Then

$$
\amalg_{\mathbf{P}^{2}-Z}(A) / \amalg_{\mathbf{P}^{2}}(A)=(\mathbf{Q} / \mathbf{Z})^{\oplus 2} .
$$

We can obtain any desired multiplicity along one of the lines, but the same multiplicity is then forced along the other line, with invariant the negative of the invariant along the first line.

2) By construction, there is a map $g: X_{2} \rightarrow \mathbf{P}^{1}$ via $X_{2} \rightarrow \mathbf{P}^{2} \times \mathbf{P}^{1} \rightarrow \mathbf{P}^{1}$. The general fibre is a non-singular quadric surface and there are four singular fibres consisting each of two components, a $\mathbf{P}^{2}$ and a scroll. The four $\mathbf{P}^{2}$ 's can be contracted to smooth points, leaving $g^{\prime}: X^{\prime} \rightarrow \mathbf{P}^{1}$, a relatively minimal quadric bundle. It is well-known that $C H^{2}\left(X^{\prime}\right)=\mathbf{Z} \oplus \operatorname{Pic} E$, where $E$ is the curve (elliptic in this case) obtained by taking the branched cover $\xi: E \rightarrow \mathbf{P}^{1}$, branched at the four points $\left\{P_{1}, \ldots, P_{4}\right\}$ where the fibres of $g^{\prime}$ are singular. For a point $P \in E$, the corresponding cycle is a line $l_{P}$ in the appropriate family of lines in the quadric $X_{\xi(P)}^{\prime}$. The curve $E$ is of course isomorphic to any of the non-singular fibres of $f_{2}: X_{2} \rightarrow \mathbf{P}^{2}$. We denote the components of $f_{2}^{-1}(C)$ by $Y_{1}, \ldots, Y_{5}, Y_{3}$ the multiple component, with $Y_{1}, Y_{2}, Y_{4}$ and $Y_{5}$ mapped to $P_{1}, P_{2}, P_{3}$ and $P_{4}$ respectively by $g$.

Now consider $\gamma \in H_{Z_{1}}^{2}\left(S, i_{*} A\right)=\operatorname{ker} \phi$. In the notation of (4.7), $G_{1}=0$ and $G_{2}=\operatorname{ker} \phi$. Let $\sigma_{0}: \mathbf{P}^{2} \rightarrow X_{2}$ be the section whose image is the $\mathbf{P}^{2}$ contained in $g^{-1}\left(P_{1}\right)$, and let $\sigma: \mathbf{P}^{2} \rightarrow X_{2}$ be the section whose image is the $\mathbf{P}^{2}$ contained in $g^{-1}\left(P_{2}\right)$. Let $S_{0}=\sigma_{0}\left(\mathbf{P}^{2}\right) \cap f^{-1}\left(Z_{1}\right)$ be the zero section in $M W\left(f^{-1}\left(Z_{1}\right) / Z_{1}\right)$, and without loss of generality, suppose $\gamma \in H_{Z_{1}}^{2}\left(\mathbf{P}^{2}, i_{*} A\right)$ is given by the section 
$S=\sigma\left(\mathbf{P}^{2}\right) \cap f^{-1}\left(Z_{1}\right)$. Then as one can see either by direct calculation or by referring to $[6]$, we find on $f^{-1}\left(Z_{1}\right)$ that

$$
2\left(S-S_{0}\right) \sim Y_{1} \cap f^{-1}\left(Z_{1}\right)-Y_{2} \cap f^{-1}\left(Z_{1}\right) .
$$

Thus, by Theorem 3.3, $\gamma$ maps in $H_{Z_{1}}^{3}(S, \mathcal{E})$ to the element represented by $(1 / 2,1 / 2$, $0,0,0)$ at each point in $Z_{1} \cap C=\left\{Q_{1}, \ldots, Q_{n}\right\}$. Following diagram (4.11), this pulls back to an element

$$
(h \otimes 1 / 2, h \otimes 1 / 2,0,0,0) \in \bigoplus_{i=1}^{5} \operatorname{ker}\left(K\left(C\left(t_{i}\right)\right)^{*} \otimes \mathbf{Q} / \mathbf{Z} \rightarrow \bigoplus_{x \in C\left(t_{i}\right)-q_{t}^{-1}(Z)} \mathbf{Q} / \mathbf{Z}\right)
$$

where $h \in K(C(t))^{*}=K\left(C\left(t_{i}\right)\right)^{*}$ is a rational function whose associated principal divisor is

$$
(h) \equiv \sum_{i=1}^{n} Q_{i} \quad \bmod 2 .
$$

(For example, we can choose $h$ so that $(h)=\sum_{i=1}^{n}(-1)^{i} Q_{i}$, since $n$ is even.) This then maps to ker $\partial_{2}^{U}$ by considering $h$ as a rational function on $Y_{i}$ via the pull-back $Y_{i} \rightarrow C\left(t_{i}\right)$. To pull this back to an element of $\operatorname{ker} \partial_{2}^{X}$, we need a rational function $g \in K\left(f^{-1}\left(Z_{1}\right)\right)^{*}$ which will together with $h$ in $K\left(Y_{1}\right)^{*}$ and $K\left(Y_{2}\right)^{*}$ yield an element of $\operatorname{ker} \partial_{2}^{X}$. To do this, on $f^{-1}\left(Z_{1}\right)$ we have, as we have already noted,

$$
2\left(S-S_{0}\right) \sim \sum_{i=1}^{n} C_{1}^{i}-C_{2}^{i}
$$

with $C_{j}^{i}=Y_{j} \cap f^{-1}\left(Q_{i}\right)$, so we take $g \in K\left(f^{-1}\left(Z_{1}\right)\right)^{*}$ so that

$$
(g)=2\left(S-S_{0}\right)+\sum_{i=1}^{n} C_{2}^{i}-C_{1}^{i}
$$

and $g \otimes 1 / 2$ then cancels the contributions of $h \otimes 1 / 2$ on $Y_{1}$ and $Y_{2}$ in $\bigoplus_{x \in X^{(2)}} \mathbf{Z} / 2 \mathbf{Z}$.

The recipe of (4.3) tells us that the torsion cycle corresponding to the collection of functions $h \in K\left(Y_{1}\right)^{*}, h \in K\left(Y_{2}\right)^{*}$ and $g \in K\left(f^{-1}\left(Z_{1}\right)\right)^{*}$ representing an element of $\operatorname{ker} \partial_{2}^{X}$ is

$$
S-S_{0}+n C_{2} / 2-n C_{1} / 2,
$$

where $C_{2}$ is the class of $C_{2}^{i}$ for any $i$. Of course, $n=2 \operatorname{deg} Z_{1}=2 d$, so we obtain

$$
\psi^{\prime}(\gamma)=\left[S-S_{0}+d C_{2}-d C_{1}\right] \in C(X / S)=(\operatorname{Pic} E)_{\text {tors }},
$$

where square brackets denote the class of the cycle in $C(X / S)$. Let $l_{i}$ be a line on the cone $g^{\prime-1}\left(P_{i}\right)$, and $\pi_{1}: X_{2} \rightarrow X^{\prime}$ be the blowing-down. Then

$$
\pi_{1 *}\left(\left[S-S_{0}+d C_{2}-d C_{1}\right]\right)=d l_{2}-d l_{1}
$$

on $X^{\prime}$. In fact, $l_{2}-l_{1}$ is a 2 -torsion cycle, so we see that $\psi^{\prime}(\gamma)=0$ if and only if $d$ is even.

Finally, we obtain

$$
\amalg_{\mathbf{P}^{2}-Z_{1}}(A) / \amalg_{\mathbf{P}^{2}}(A)= \begin{cases}\mathbf{Z} / 2 \mathbf{Z} \oplus \mathbf{Z} / 2 \mathbf{Z} & \text { if } \operatorname{deg} Z_{1} \text { is even, } \\ 0 & \text { if } \operatorname{deg} Z_{1} \text { is odd. }\end{cases}
$$

We next consider $Z_{2}$. Since for this situation we showed $\operatorname{ker} \phi=H_{l}^{2}\left(S, i_{*} A\right)$, we can replace $Z_{2}$ with $l$. We have $f^{-1}(l)=l \times E, p_{2}: l \times E \rightarrow E$ the second 
projection. We have $\sigma_{0}\left(S_{2}\right) \cap f^{-1}(l)=p_{2}^{-1}\left(P_{0}\right)$ for some $P_{0} \in E$. Given a section $S \in M W\left(f^{-1}(l) / l\right)_{\text {tors }}$, we have $S=p_{2}^{-1}(P)$ for some $P \in E$, and the corresponding cycle class in $C\left(X_{2}^{\prime} / S_{2}\right)=C H_{2}\left(X_{2}^{\prime}\right)_{\text {tors }}$ is $\left[S-S_{0}\right]$ by Theorem 4.3. It is easy to see that $\left[S-S_{0}\right]$ is not rationally equivalent to zero unless $S=S_{0}$ by restricting this cycle to $f^{-1}(l)$. Thus $\operatorname{ker} \phi=0$, and

$$
\amalg_{\mathbf{P}^{2}-l}(A)=\amalg_{\mathbf{P}^{2}}(A) .
$$

We obtain no multiple fibres along $l$.

3) By running Mori's minimal model algorithm on $X_{3}$, one finds that $X_{3}$ is obtained by blowing up a $\mathbf{P}^{1}$ bundle over the scroll $F_{1}$ in a series of points and $\mathbf{P}^{1}$ 's. Thus $C H^{2}(X)_{\text {tors }}=0$, and $C(X / S)=0$. Hence there is no further obstruction. We omit the details of this analysis.

4) Similarly, $X_{4}$ can be obtained by blowing up points and $\mathbf{P}^{1}$ 's and some flops from $\mathbf{P}^{3}$ blown up in 8 points, except for a final blow-up of the singular curve in the divisor over the $I_{1}$ curve, this being a curve $C$ of genus 3. From this description, we see that $C H^{2}\left(X_{4}\right)_{\text {tors }}=(\operatorname{Pic} C)_{\text {tors }}$, and $C(X / S)=0$. Again there is no obstruction. For details on the blowups, see [11], where the blowups of cuspidal points are analysed.

5) We add another example. Suppose $f: X \rightarrow S$ is a modified Miranda model with a curve $C \subseteq S$ with $C^{2}=0$ of fibre type $I_{M}, M>0$, and $f^{-1}(C)$ a product of $C$ and a cycle of $M^{\prime}$ rational curves. Then it is easy to check that $H_{C}^{2}\left(S, i_{*} A\right)=$ $\operatorname{ker} \phi=\mathbf{Q} / \mathbf{Z}$ and that $C H^{1}\left(f^{-1}(C)\right)_{\text {tors }}=\left(C H^{1}(C)_{\text {tors }}\right)^{M^{\prime}}$. In fact sequence (4.2) gives

$$
0 \rightarrow \mathbf{Q} / \mathbf{Z} \rightarrow H_{f^{-1}(C)}^{3}(X, \mathbf{Q} / \mathbf{Z}) \rightarrow C H^{1}\left(f^{-1}(C)\right)_{\text {tors }} \rightarrow 0
$$

and this $\mathbf{Q} / \mathbf{Z}$ term maps to zero in $H^{3}(X, \mathbf{Q} / \mathbf{Z})$. Thus we see that

$$
\amalg_{S-C}(A) / \amalg_{S}(A)=\mathbf{Q} / \mathbf{Z}
$$

completely independently of any other geometry of $X$.

\section{Appendix: The calculations for $I_{m}, m \geq 1$}

We begin with some auxiliary geometric results about the models of elliptic threefolds we use. We are assuming that $f: X \rightarrow S$ is a modified Miranda model, which, as we mentioned in $\S 1$, has $\Sigma$ s.n.c. and contains only a small number of possible collisions. In particular, we have no collisions $I_{M_{1}}+I_{M_{2}}$ with $M_{1}$ and $M_{2}$ odd, and, as mentioned earlier, we have blown up the minimal model so as to avoid divisors which are not normal over curves of fibre type $I_{M}, M$ odd. Under these conditions, if $S$ is projective, then we have strong conditions on these curves of the discriminant locus.

We also note that the Miranda model is hardly unique; it depends on the order in which one resolves the singular loci of the Weierstrass model over various curves of $\Sigma$. In our case, the resolution we described assumes that all singularities over curves of type $I_{M}$ with $M$ even are resolved before those with $M$ odd; in Table 1, we resolve over $\Sigma_{2}$ before $\Sigma_{1}$.

Theorem A.1. Let $C \subseteq \Sigma$ be a complete curve of fibre type $I_{M}$, case I, colliding with curves $D_{1}, \ldots, D_{r}$ of fibre type $I_{M_{i}}, 1 \leq i \leq r$, which are resolved before $C$, and curves $D_{r+1}, \ldots, D_{s}$ of fibre type $I_{M_{i}}, r+1 \leq i \leq s$, which are resolved after 
C. Then one obtains

$$
\sum_{j=1}^{s} M_{j}=-M C^{2}
$$

and, for $M$ even, with $f^{-1}(C)=Y_{1} \cup \cdots \cup Y_{M}$ as in Table 1,

$$
\left(C_{i}^{2}\right)_{Y_{i+1}}= \begin{cases}i C^{2}+\sum_{j=1}^{r} \frac{M_{j}}{2}, & 1 \leq i \leq M / 2, \\ i C^{2}+\sum_{j=1}^{r} \frac{M_{j}}{2}+\sum_{j=r+1}^{s} M_{j}, & M / 2+1 \leq i \leq M-1\end{cases}
$$

(here $\left(C_{i}^{2}\right)_{Y_{i+1}}$ denotes the self-intersection of the curve $C_{i}$ considered as a curve on the surface $\left.Y_{i+1}\right)$ and

$$
\left(C_{1}^{2}\right)_{Y_{1}}=\left(C_{M}^{2}\right)_{Y_{1}}=-\sum_{j=1}^{r} \frac{M_{j}}{2} .
$$

For $M$ odd, with $f^{-1}(C)=Y_{1} \cup \cdots \cup Y_{M+1}$, one has

$$
\left(C_{i}^{2}\right)_{Y_{i+1}}= \begin{cases}i C^{2}+\sum_{j=1}^{r} \frac{M_{j}}{2}, & 1 \leq i \leq(M-1) / 2, \\ M C^{2}+\sum_{j=1}^{r} M_{j}, & i=(M+1) / 2, \\ (i-1) C^{2}+\sum_{j=1}^{r} \frac{M_{j}}{2}, & (M+3) / 2 \leq i \leq M,\end{cases}
$$

and

$$
\left(C_{1}^{2}\right)_{Y_{1}}=\left(C_{M+1}^{2}\right)_{Y_{1}}=-\sum_{j=1}^{r} \frac{M_{j}}{2}
$$

Proof. Observe that by our assumptions on ordering, $M_{i}$ is even for $1 \leq i \leq r$, and if $M$ is odd, then $r=s$. Put

$$
M^{\prime}:= \begin{cases}M, & M \text { even } \\ M+1, & M \text { odd }\end{cases}
$$

Consulting Table 1, the geometry of $Y_{1}$ is as shown in Figure A.1 where $\sigma_{0}$ is the zero-section of $f: X \rightarrow S$. From this, it is clear that on $Y_{1}$

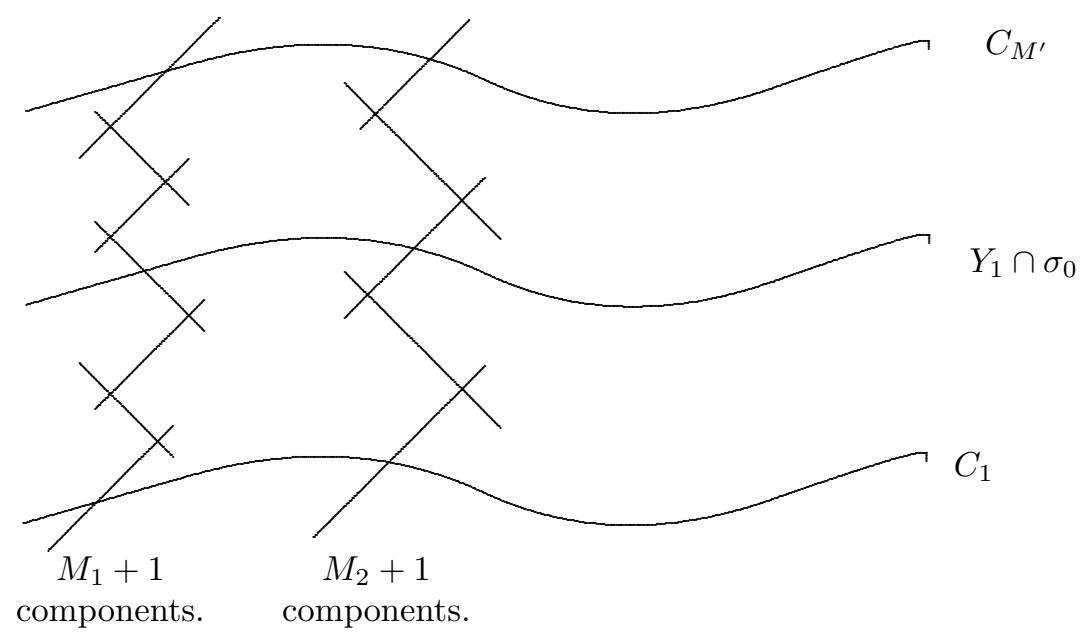

FIGURE A.1 


$$
C_{M^{\prime}}^{2}=C_{1}^{2}=-\sum_{i=1}^{r} \frac{M_{i}}{2}
$$

since if we contract all components of $f: Y_{1} \rightarrow C$ not intersecting $Y_{1} \cap \sigma_{0}$, we obtain a scroll with three disjoint sections, each of which must then have self-intersection 0 . We can write

$$
f^{*} C=\sum_{i=1}^{M^{\prime}} a_{i} Y_{i}
$$

where

$$
a_{i}= \begin{cases}2, & M \text { odd and } i=(M+3) / 2, \\ 1, & \text { otherwise }\end{cases}
$$

Now

$$
\begin{aligned}
C^{2}=f^{*} C \cdot C_{i} & =\sum_{j=1}^{M^{\prime}} a_{j} Y_{j} \cdot C_{i} \\
& =a_{i} Y_{i} \cdot C_{i}+a_{i+1} Y_{i+1} \cdot C_{i} \\
& =a_{i} Y_{i} \cdot\left(s_{i+1}\right)_{*} C_{i}+a_{i+1} Y_{i+1} \cdot\left(s_{i}\right)_{*} C_{i} \\
& =a_{i}\left(\left(s_{i+1}^{*} Y_{i}\right) \cdot C_{i}\right)_{Y_{i+1}}+a_{i+1}\left(\left(s_{i}^{*} Y_{i+1}\right) \cdot C_{i}\right)_{Y_{i}}
\end{aligned}
$$

where $s_{i}: Y_{i} \rightarrow X$ is the inclusion. Now $Y_{i} \cdot Y_{i+1}=C_{i}$ (or, if $M^{\prime}=2, Y_{1} \cdot Y_{2}=$ $C_{1}+C_{2}$, with $C_{1}, C_{2}$ disjoint), so we get

$$
C^{2}=a_{i}\left(C_{i}^{2}\right)_{Y_{i+1}}+a_{i+1}\left(C_{i}^{2}\right)_{Y_{i}} .
$$

We also have

$$
\left(C_{i-1}^{2}\right)_{Y_{i}}+\left(C_{i}^{2}\right)_{Y_{i}}=0
$$

whenever $Y_{i}$ is a geometrically ruled surface (which is true for $i \neq 1$ if $M$ is odd and $i \neq 1, M / 2+1$ if $M$ is even), since $C_{i-1}$ and $C_{i}$ are disjoint. By using (A.2) and (A.3) repeatedly, for $M$ odd, we see that

$$
\left(C_{i}^{2}\right)_{Y_{i+1}}= \begin{cases}i C^{2}+\sum_{j=1}^{r} \frac{M_{j}}{2}, & 1 \leq i \leq(M-1) / 2, \\ M C^{2}+\sum_{j=1}^{r} M_{j}, & i=(M+1) / 2, \\ (i-1) C^{2}+\sum_{j=1}^{r} \frac{M_{j}}{2}, & (M+3) / 2 \leq i \leq M,\end{cases}
$$

and

$$
\left(C_{M^{\prime}}^{2}\right)_{Y_{1}}=M C^{2}+\sum_{j=1}^{r} \frac{M_{j}}{2}=-\sum_{j=1}^{r} \frac{M_{j}}{2}
$$

by (A.1), and hence $M C^{2}=-\sum_{j=1}^{r} M_{j}$ as promised.

When $M$ is even, (A.4) remains valid for $1 \leq i \leq M / 2$. The geometry of $Y_{M / 2+1}$ is then as shown in Figure A.2. From this, one sees on $Y_{M / 2+1}$ that

$$
C_{M / 2}^{2}+\sum_{j=r+1}^{s} M_{j}+C_{M / 2+1}^{2}=0
$$




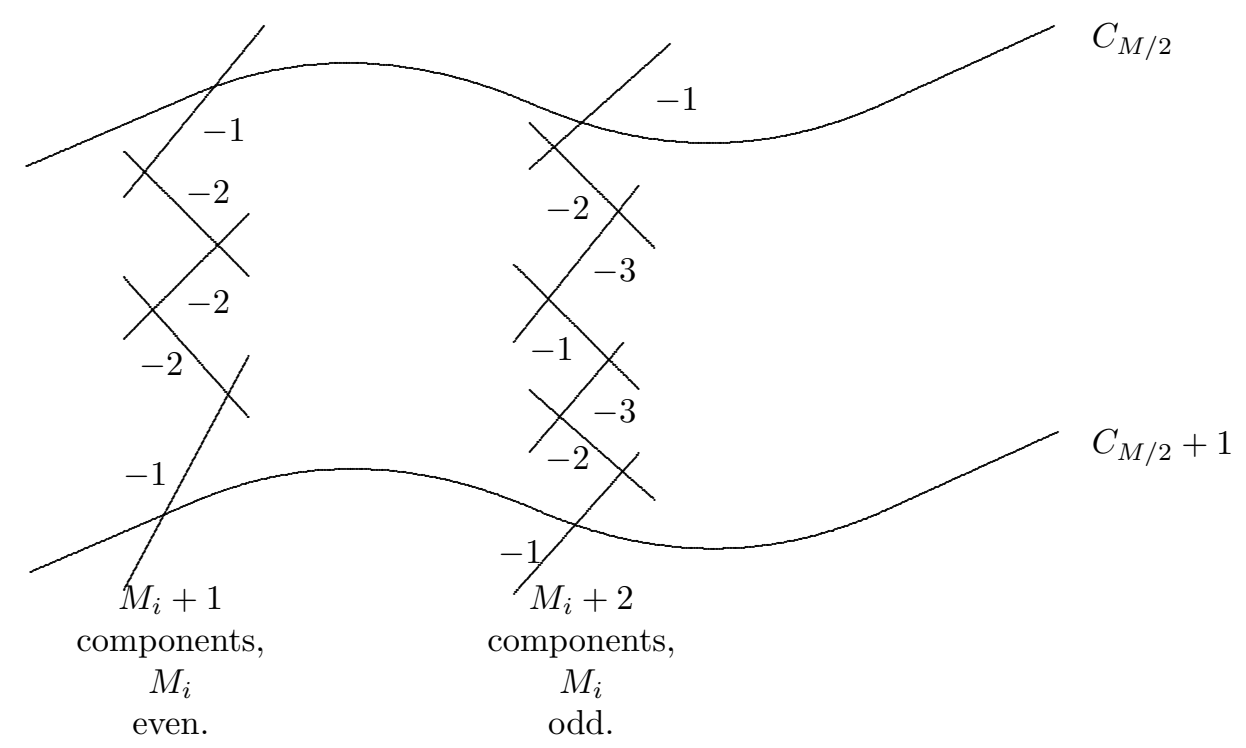

Figure A.2

Using this, one continues, seeing that

$$
\left(C_{i}^{2}\right)_{Y_{i+1}}=i C^{2}+\sum_{j=1}^{r} \frac{M_{j}}{2}+\sum_{j=r+1}^{s} M_{j}
$$

and

$$
\left(C_{M}^{2}\right)_{Y_{1}}=M C^{2}+\sum_{j=1}^{r} \frac{M_{j}}{2}+\sum_{j=r+1}^{s} M_{j}=-\sum_{j=1}^{r} \frac{M_{j}}{2},
$$

so again one has

$$
M C^{2}=-\sum_{j=1}^{s} M_{j}
$$

Theorem A.2. Let $C \subseteq \Sigma$ be a complete curve of fibre type $I_{M}$, of case $I^{*}$, colliding with curves $D_{1}, \ldots, D_{s}$ of fibre type $I_{M_{i}}, 1 \leq i \leq s$, with $D_{1}, \ldots, D_{r}$ resolved before $C$ and $D_{r+1}, \ldots, D_{s}$ resolved after $C$, and also colliding with curves $B_{1}, \ldots, B_{u}$ of fibre type $I_{N_{i}}^{*}, 1 \leq i \leq u$. Then $u$ is even and

$$
-M C^{2}=\sum_{j=1}^{s} M_{j}+\sum_{j=1}^{u} N_{j}
$$

In addition, if one puts

$$
\eta_{i}= \begin{cases}N_{i}+2 & \text { if } N_{i} \text { is even, } M>1 \\ N_{i}+1 & \text { if } N_{i} \text { is odd, } M>1 \\ N_{i}+3 & \text { if } M=1\end{cases}
$$


and $f^{-1}(C)=Y_{1} \cup \cdots \cup Y_{M / 2+1}$ if $M$ is even and $f^{-1}(C)=Y_{1} \cup \cdots \cup Y_{(M+3) / 2}$ if $M$ is odd as in Table 2, then one has

$$
\left(C_{1}^{2}\right)_{Y_{1}}=2 u-\sum_{j=1}^{r} M_{j}-\sum_{j=1}^{u} \eta_{j}
$$

and

$$
\left(C_{i}^{2}\right)_{Y_{i+1}}=2 i C^{2}-2 u+\sum_{j=1}^{r} M_{j}+\sum_{j=1}^{u} \eta_{j}
$$

for

$$
\begin{cases}1 \leq i \leq M / 2 & \text { if } M \text { is even, } \\ 1 \leq i \leq(M-1) / 2 & \text { if } M \text { is odd }\end{cases}
$$

and if $M$ is odd,

$$
\left(C_{(M+1) / 2}^{2}\right)_{Y_{(M+3) / 2}}=2 u \text {. }
$$

If $M$ is odd, then $Y_{(M+3) / 2} \cong \mathbf{P}_{C}(\mathcal{E})$ where $\mathcal{E}$ is a rank 2 vector bundle on $C$ with $c_{1}(\mathcal{E})=C^{2}-u / 2$.

Proof. Letting $\sigma_{0} \subseteq X$ be the zero section of $f: X \rightarrow S$, one sees that

$$
\begin{aligned}
\left(\left(\sigma_{0} \cap Y_{1}\right)^{2}\right)_{Y_{1}} & =\sigma_{0}^{2} \cdot Y_{1} \\
& =\sigma_{0}^{2} \cdot f^{*} C \\
& =f_{*} \sigma_{0}^{2} \cdot C \\
& =-L . C
\end{aligned}
$$

where $\mathcal{O}_{S}(L)$ is the line bundle $\mathcal{L}$ defining the Weierstrass model (see $\S 1$ ).

Now $\Sigma \sim 12 L$, and thus

$$
12 C . L=M C^{2}+\sum_{j=1}^{s} M_{j}+\sum_{j=1}^{u}\left(N_{j}+6\right),
$$

as $C$ appears with multiplicity $M$ in $\Sigma, D_{j}$ with multiplicity $M_{j}$ and $B_{j}$ with multiplicity $N_{j}+6$ (see for example [16] ). But since $\Sigma$ is s.n.c., the $J$ function induces a morphism $J: S \rightarrow \mathbf{P}^{1}$, and $\Sigma \sim S_{\infty}+\sum n_{i} \Sigma_{i}$ where $S_{\infty}$ is the fibre over $\infty \in \mathbf{P}^{1}$ and the $\Sigma_{i}$ are the components of $\Sigma, n_{i}=0,2,3,4,6,8,9$ or 10 in the cases that $\Sigma_{i}$ is of fibre type $I_{a}, I I, I I I, I V, I_{a}^{*}, I V^{*}, I I I^{*}$ or $I I^{*}$ respectively. We thus have

$$
C . \Sigma=S_{\infty} . C+\sum n_{i} \Sigma_{i} . C=0+\sum_{j=1}^{u} 6=6 u
$$

Thus

$$
6 u=M C^{2}+\sum_{j=1}^{n} M_{j}+\sum_{j=1}^{u}\left(N_{j}+6\right)
$$

and

$$
-M C^{2}=\sum_{j=1}^{s} M_{j}+\sum_{j=1}^{u} N_{j},
$$




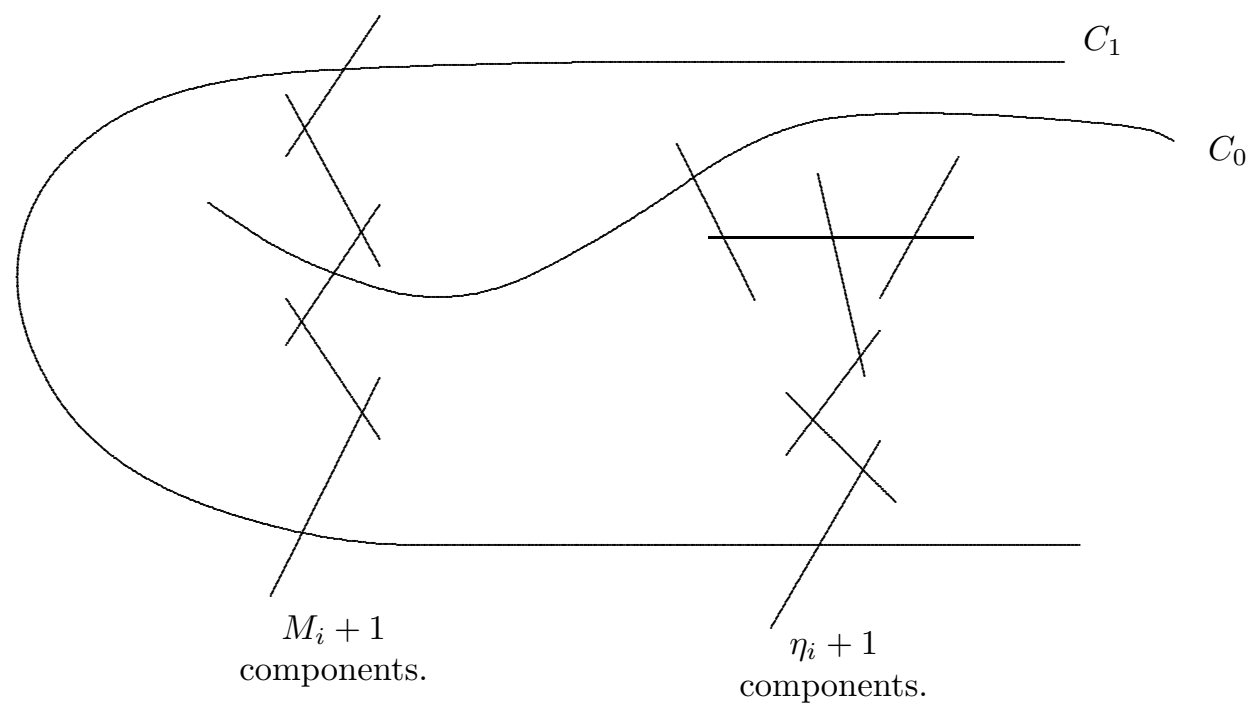

FiguRe A.3

proving the first claim. In particular,

$$
\left(\left(\sigma_{0} \cap Y_{1}\right)^{2}\right)_{Y_{1}}=-u / 2,
$$

and $u$ is even.

We now look at the picture for $Y_{1}$ (Figure A.3).

From this diagram one sees that, if $\bar{Y}_{1}$ is the surface obtained by contracting all components of fibres of $Y_{1} \rightarrow C$ not intersecting $C_{0}=\sigma_{0} \cap Y_{1}$, and if $\bar{C}_{1}$ is the image of $C_{1}$ in $\bar{Y}_{1}$, we can write

$$
\bar{C}_{1} \equiv 2 C_{0}+a f
$$

where $\equiv$ denotes numerical equivalence, $f$ the class of a fibre. Since $\bar{C}_{1} \cdot C_{0}=0$, we see that $a=-2 C_{0}^{2}$, whence $\bar{C}_{1}^{2}=-4 C_{0}^{2}=2 u$, and so

$$
\left(C_{1}^{2}\right)_{Y_{1}}=2 u-\sum_{j=1}^{r} M_{j}-\sum_{j=1}^{u} \eta_{j} .
$$

One then obtains, analogously to the derivation of (A.2), keeping in mind that the curves $C_{i}$ map 2-1 to $C$, that

$$
2 C^{2}=a_{i}\left(C_{i}\right)_{Y_{i+1}}^{2}+a_{i+1}\left(C_{i}^{2}\right)_{Y_{i}} .
$$

Using (A.6) and (A.3), we get

$$
\left(C_{i}^{2}\right)_{Y_{i+1}}=2 i C^{2}-2 u+\sum_{j=1}^{r} M_{j}+\sum_{j=1}^{u} \eta_{j}
$$

for

$$
\begin{cases}1 \leq i \leq M / 2 & \text { if } M \text { is even } \\ 1 \leq i \leq(M-1) / 2 & \text { if } M \text { is odd }\end{cases}
$$




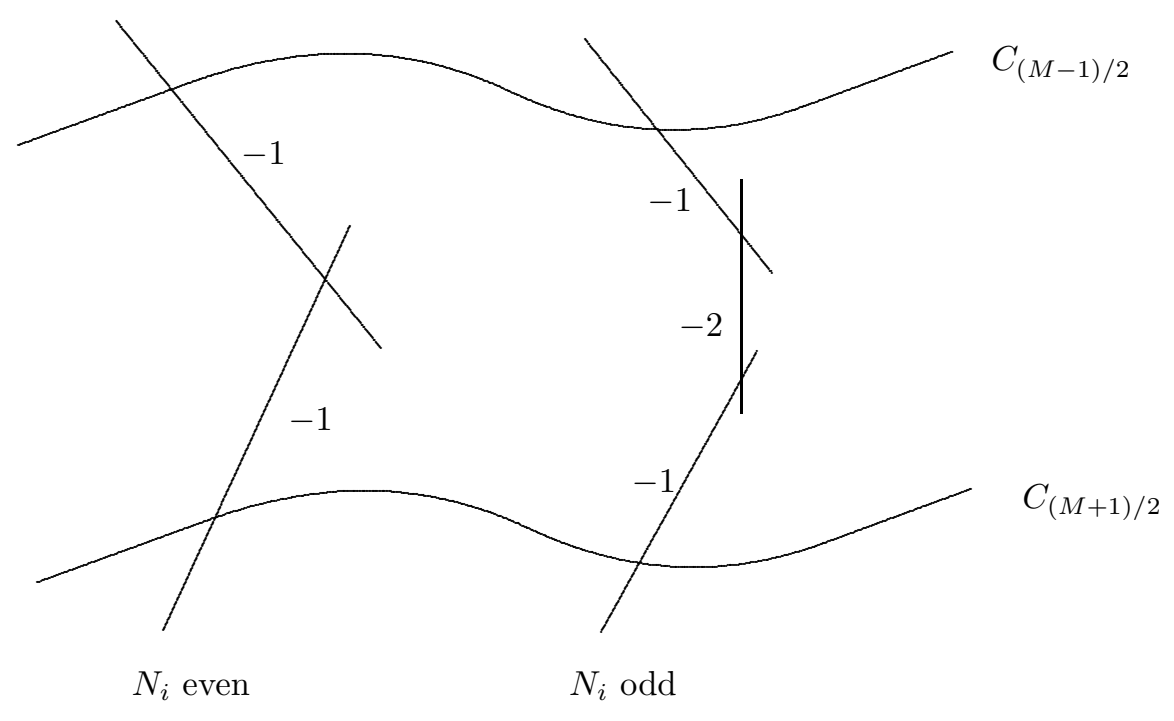

FiguRE A.4

and

$$
\begin{aligned}
\left(C_{1}^{2}\right)_{Y_{2}} & =2 C^{2}-4 u+2\left(\sum_{j=1}^{r} M_{j}+\sum_{j=1}^{u} \eta_{j}\right) \\
& =2 u
\end{aligned}
$$

if $M=1$, using the first equality of the theorem. If $M$ is odd, $M>1$, then $Y_{(M+1) / 2}$ looks like Figure A.4 so

$$
\left(C_{(M+1) / 2}^{2}\right)_{Y_{(M+1) / 2}}=-\left(\# N_{i} \text { even }+2\left(\# N_{i} \text { odd }\right)\right)-\left(C_{(M-1) / 2}^{2}\right)_{Y_{(M+1) / 2}}
$$

and thus

$$
\begin{aligned}
\left(C_{(M+1) / 2}^{2}\right)_{Y_{(M+3) / 2}} & =2 C^{2}-2\left(C_{(M+1) / 2}^{2}\right)_{Y_{(M+1) / 2}} \\
& =2 M C^{2}-4 u+2\left(\sum_{j=1}^{r} M_{j}+\sum_{j=1}^{u}\left(\eta_{j}+2\right)-\# N_{j} \text { even }\right) \\
& =2 M C^{2}-4 u+2\left(\sum_{j=1}^{r} M_{j}+\sum_{j=1}^{u}\left(N_{j}+3\right)\right)
\end{aligned}
$$

by the definition of $\eta_{j}$. Using the first equality of the theorem, this reduces to the form given.

For the last statement, $Y_{(M+3) / 2}$ was the exceptional divisor of the blowup of a curve $C^{\prime}$ in $X^{\prime}$, the original Miranda model, with $f^{\prime}: X^{\prime} \rightarrow S$ mapping $C^{\prime}$ isomorphically to $C$. We thus have $Y_{(M+3) / 2} \cong \mathbf{P}_{C}\left(\mathcal{N}_{C^{\prime} / X^{\prime}}\right)$, where $\mathcal{N}_{C^{\prime} / X^{\prime}}$ is the 
normal bundle of $C^{\prime}$ in $X^{\prime}$. Now

$$
\begin{aligned}
c_{1}\left(\mathcal{N}_{C^{\prime} / X^{\prime}}\right) & =c_{1}\left(\left.\mathcal{T}_{X^{\prime}}\right|_{C^{\prime}}\right)-c_{1}\left(\mathcal{T}_{C^{\prime}}\right) \\
& =-K_{X^{\prime}} \cdot C^{\prime}+2 g_{C}-2 \\
& =-f^{\prime *}\left(K_{S}+L\right) \cdot C^{\prime}+2 g_{C}-2 \\
& =-K_{S} \cdot C-L \cdot C+2 g_{C}-2 \\
& =2-2 g_{C}+C^{2}-C \cdot L+2 g_{C}-2 \\
& =C^{2}-u / 2 .
\end{aligned}
$$

We now move on to the proofs of Theorems 3.4 and 3.6. We first explain how to carry out the calculation in general. Let $f: X \rightarrow S$ satisfy the additional Hypothesis. We will assume that $Z$ consists of precisely those components of $\Sigma$ of fibre type $I_{M}$. Put $U=S-\Sigma^{\prime}$ where $\Sigma^{\prime}$ consists of all components of $\Sigma$ contained in $S_{0}$ and $S_{1}$, the fibres of the $J$-map $J: S \rightarrow \mathbf{P}^{1}$ over $0,1 \in \mathbf{P}^{1}$. If we put $\Delta=\Sigma-\Sigma^{\prime}$, it follows from Miranda's analysis that $f^{-1}(\Delta)$ has simple normal crossings in $X_{U}=X \times{ }_{S} U$. Furthermore, the only components of $\Delta$ which are complete are of fibre type $I_{M}, M \geq 1$, or $I_{M}^{*}, M \geq 1$, as these are the only components of $\Sigma$ contained in fibres of $J$ other than $S_{0}$ and $S_{1}$. We thus have $H^{3}(U, \mathcal{E})=H_{\Delta}^{3}(U, \mathcal{E})=\bigoplus_{t \in I} H^{3}\left(U, \mathcal{E}_{t}\right)$, where $I \subseteq S^{(1)}$ is the set of generic points of curves of fibre type $I_{M}$ or $I_{M}^{*}, M \geq 1$. Furthermore, by Theorem 2.11 and Remark 2.12, $H_{Z}^{2}\left(S, i_{*} A\right)=H_{\Delta}^{2}\left(U, i_{*} A\right)$. Thus we need to interpret the map

$$
H_{\Delta}^{2}\left(U, i_{*} A\right) \stackrel{\phi_{1}}{\longrightarrow} H_{\Delta}^{3}(U, \mathcal{E}) .
$$

By Prop. 2.2, since $\underline{H}_{\Delta}^{i}(U, \mathcal{E})=0, \forall i>0$, we have $\underline{H}_{\Delta}^{2}\left(U, i_{*} A\right)=\underline{H}_{\Delta}^{2}\left(U, P_{X / S}\right)$ and thus $H_{\Delta}^{2}\left(U, i_{*} A\right)=H^{0}\left(U, \underline{H}_{\Delta}^{2}\left(U, P_{X / S}\right)\right)$. By Proposition 1.6, we then have exact sequences

$$
0 \longrightarrow \bigoplus_{i<j}(\mathbf{Q} / \mathbf{Z})_{Z_{i} \cap Z_{j}} \stackrel{\alpha}{\longrightarrow} \bigoplus_{(i, j)<\left(i^{\prime}, j^{\prime}\right)} k_{*}(\mathbf{Q} / \mathbf{Z})_{Y_{i}^{j} \cap Y_{i^{\prime}}^{j^{\prime}}} \longrightarrow \mathcal{F} \longrightarrow 0
$$

and

$$
0 \rightarrow H_{\Delta}^{2}\left(U, i_{*} A\right) \stackrel{\beta}{\longrightarrow} H^{0}(\mathcal{F}) \rightarrow \bigoplus_{i, j} H^{0}\left(U, R^{2} k_{*}(\mathbf{Q} / \mathbf{Z})_{Y_{i}^{j}}\right)
$$

where the $Y_{i}^{j}$ are the components over the $Z_{i}$ 's, the components of $\Delta$. Of course,

$$
H_{\Delta}^{3}(U, \mathcal{E})=H^{3}(U, \mathcal{E})=\bigoplus_{i} \frac{\bigoplus_{j} H^{2}\left(C_{i}^{j}, \mathbf{Q} / \mathbf{Z}\right)}{H^{2}\left(Z_{i}, \mathbf{Q} / \mathbf{Z}\right)}
$$

where $Y_{i}^{j} \rightarrow C_{i}^{j} \rightarrow Z_{i}$ is the Stein factorization, and thus there is a natural inclusion of $H_{\Delta}^{3}(U, \mathcal{E})$ in

$$
\bigoplus_{i} \frac{\bigoplus_{j} H^{2}\left(Y_{i}^{j}, \mathbf{Q} / \mathbf{Z}\right)}{H^{2}\left(Z_{i}, \mathbf{Q} / \mathbf{Z}\right)}
$$


The diagram

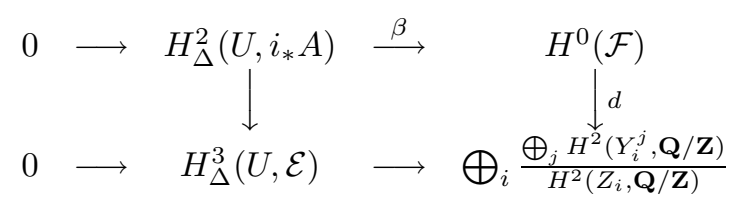

is commutative, where $d$ is the map induced by

$$
d: \bigoplus_{(i, j)<\left(i^{\prime}, j^{\prime}\right)} H^{0}\left(Y_{i}^{j} \cap Y_{j^{\prime}}^{i^{\prime}}, \mathbf{Q} / \mathbf{Z}\right) \rightarrow \bigoplus_{(i, j)} H^{2}\left(Y_{i}^{j}, \mathbf{Q} / \mathbf{Z}\right)
$$

of Proposition 1.5. Thus we use the map $d$ to understand $\phi_{1}$.

Proof of Theorem 3.4. We retain the assumptions on ordering of resolution of the Weierstrass model made in Theorem A.1. To calculate $\phi_{t}(\gamma)$, we need to know what the image of $\gamma$ is in

$$
H^{0}(\mathcal{F})=\frac{\bigoplus_{(i, j)<\left(i^{\prime}, j^{\prime}\right)} H^{0}\left(Y_{i}^{j} \cap Y_{i^{\prime}}^{j^{\prime}}, \mathbf{Q} / \mathbf{Z}\right)}{\bigoplus_{i<j} H^{0}\left(Z_{i} \cap Z_{j}, \mathbf{Q} / \mathbf{Z}\right)}
$$

under the map $\beta$. This we already know from our local calculations. Indeed, the curves $Y_{i}^{j} \cap Y_{i^{\prime}}^{j^{\prime}}$ of concern to us are $C_{1}, \ldots, C_{M^{\prime}}$ with $C_{i}=Y_{i} \cap Y_{i+1}, C_{M^{\prime}}=Y_{M^{\prime}} \cap$ $Y_{1}$, and the curves $f_{1}^{j}, \ldots, f_{M^{\prime}+M_{j}}^{j}$ over the collision points $I_{M}+I_{M_{j}}, 1 \leq j \leq s$. For our purposes, we can assume that there are no other curves of the form $Y_{i}^{j} \cap Y_{i^{\prime}}^{j^{\prime}}$ than those mentioned, as we are only interested in what is going on over $C$. A representative of $\beta(\gamma)$ in the $H^{0}$ of these curves can be easily read off from the proofs of Theorems 2.9 and 2.10. Let $a_{i}^{j}, 1 \leq j \leq s, 1 \leq i \leq M^{\prime}+M_{j}$, be the image of a representative of $\beta(\gamma)$ in $H^{0}\left(f_{i}^{j}, \mathbf{Q} / \mathbf{Z}\right)$; these values are given in the proof of Theorem 2.9, where they are written $a_{i}$, since there we only deal with one collision. Since we are obtaining an element in $H^{0}(\mathcal{F})$, we can choose a representative with $a_{1}^{j}=0 \forall j$. Thus we obtain an element $\gamma^{\prime}$ in $\bigoplus_{i, j} H^{0}\left(f_{i}^{j}, \mathbf{Q} / \mathbf{Z}\right) \oplus \bigoplus_{i} H^{0}\left(C_{i}, \mathbf{Q} / \mathbf{Z}\right)$ of the form $\left(a_{1}^{1}, \ldots, a_{M^{\prime}+M_{j}}^{s}, b, \ldots, b,-b\right)$, which is the representative for $\beta(\gamma)$ in $\bigoplus_{(i, j)<\left(i^{\prime}, j^{\prime}\right)} H^{0}\left(Y_{i}^{j} \cap Y_{i^{\prime}}^{j^{\prime}}, \mathbf{Q} / \mathbf{Z}\right)$.

Fix one of the components of $f^{-1}(C)$, say, $Y_{1}$. We want to calculate the image of $\gamma^{\prime}$ in $H^{2}\left(Y_{1}, \mathbf{Q} / \mathbf{Z}\right)$ under the first component of the map $d$. Call this image $\gamma_{1}^{\prime}$. We know that $\gamma_{1}^{\prime}$ must be a pull-back of an element in $H^{2}(C, \mathbf{Q} / \mathbf{Z})=\mathbf{Q} / \mathbf{Z}$ via the map $k: Y_{1} \rightarrow C$, and if we choose a section of $k$, say $C_{1}$, then if $C_{1} \cdot \gamma_{1}^{\prime}=\gamma_{1}^{\prime \prime}$, then $\gamma_{1}^{\prime}=k^{*} \gamma_{1}^{\prime \prime}$, thinking of $\gamma_{1}^{\prime \prime}$ as an element of $H^{2}(C, \mathbf{Q} / \mathbf{Z})$. Thus, since $C_{1}$ intersects only $C_{1}$ and the $f_{1}^{j}, 1 \leq j \leq r$, among the curves listed above (we're doing the odd case first), we get that

$$
\gamma_{1}^{\prime \prime} \in H^{2}(C, \mathbf{Q} / \mathbf{Z}) \subseteq H^{2}\left(Y_{1}, \mathbf{Q} / \mathbf{Z}\right)
$$

is

$$
\begin{aligned}
\gamma_{1}^{\prime \prime}=C_{1} \cdot\left(b C_{1}+\sum_{j=1}^{r} a_{1}^{j} f_{1}^{j}\right) & =b\left(C_{1}^{2}\right)_{Y_{1}}+\sum_{j=1}^{r} a_{1}^{j} \\
& =-\sum_{j=1}^{r} \frac{M_{j} b}{2}
\end{aligned}
$$

by Theorem A.1 and $a_{1}^{j}=0$ for all $j$. 
Continuing the same strategy, using the section $C_{i}$ on the surface $Y_{i+1}$ to intersect the image in $H^{2}\left(Y_{i+1}, \mathbf{Q} / \mathbf{Z}\right)$, we get

$$
\begin{aligned}
Y_{i+1}, 1 \leq i \leq(M-1) / 2: \gamma_{i+1}^{\prime \prime} & =C_{i} \cdot\left(-b C_{i}+\sum_{j=1}^{r} a_{i+1}^{j} f_{i+1}^{j}\right) \\
& =-b\left(C_{i}^{2}\right)_{Y_{i+1}}+\sum_{j=1}^{r} a_{i+1}^{j} \\
& =-i b C^{2}-\sum_{j=1}^{r} \frac{M_{j} b}{2}-\sum_{j=1}^{r} i c_{j} .
\end{aligned}
$$

Here $C_{i}$ has a coefficient of $-b$ by our sign convention for the map $d$ in Proposition 1.5, and the value for $a_{i}^{j}$ is obtained from the proof of Proposition 2.9.

$$
\begin{aligned}
& Y_{(M+3) / 2}: \gamma_{(M+3) / 2}^{\prime \prime}=-b\left(C_{(M+1) / 2}^{2}\right)_{Y_{(M+3) / 2}}+\sum_{j=1}^{r} a_{(M+3) / 2}^{j} \\
&=2\left(-\frac{b M}{2} C^{2}-\sum_{j=1}^{r} \frac{M_{j} b}{2}-\sum_{j=1}^{r} \frac{M c_{j}}{2}\right) . \\
& Y_{i+1},(M+3) / 2 \leq i \leq M: \gamma_{i+1}^{\prime \prime}=-b\left(C_{i}^{2}\right)_{Y_{i+1}}+\sum_{j=1}^{r} a_{i+1}^{j} \\
&=-(i-1) b C^{2}-\sum_{j=1}^{r} \frac{M_{j} b}{2}-\sum_{j=1}^{r}(i-1) c_{j} .
\end{aligned}
$$

Thus we get an element $\left(\gamma_{i}^{\prime \prime}\right)_{1 \leq i \leq M^{\prime}} \in(\mathbf{Q} / \mathbf{Z})^{M^{\prime}}=\bigoplus_{i=1}^{M^{\prime}} H^{2}(C, \mathbf{Q} / \mathbf{Z})$; since

$$
H^{2}\left(S, \mathcal{E}_{t}\right)=\operatorname{coker}\left(H^{2}(C, \mathbf{Q} / \mathbf{Z}) \longrightarrow \bigoplus_{i=1}^{M^{\prime}} H^{2}(C, \mathbf{Q} / \mathbf{Z})\right),
$$

we are dividing this out by $\mathbf{Q} / \mathbf{Z}$ with the map $\mathbf{Q} / \mathbf{Z} \rightarrow(\mathbf{Q} / \mathbf{Z})^{M^{\prime}}$ given by

$$
a \mapsto(a, a, \ldots, a, 2 a, a, \ldots, a)
$$

where the $2 a$ occurs in the $Y_{(M+3) / 2}$ component. Thus we can adjust $\gamma^{\prime \prime}$ by any such amount. Using $a=\sum_{j=1}^{r} M_{j} b / 2$, we obtain, with $\alpha=-b C^{2}-\sum_{j=1}^{r} c_{j}$,

$$
\begin{array}{r}
Y_{i}, 1 \leq i \leq(M+1) / 2:(i-1) \alpha, \\
Y_{(M+3) / 2}: M \alpha, \\
Y_{i},(M+5) / 2 \leq i \leq M+1:(i-2) \alpha .
\end{array}
$$

For $M$ even, we follow the same strategy, but have to keep in mind that for $r+1 \leq j \leq s$, the $Y_{i}^{\prime}$ 's play the role of the $Y_{i}^{\prime}$ 's in Table 1 . We have

$$
\begin{gathered}
Y_{1}: b\left(C_{1}^{2}\right)_{Y_{1}}+\sum_{j=1}^{r} a_{1}^{j}-\sum_{j=r+1}^{s} a_{1+M / 2+M_{j}^{\prime}}^{j} \\
=-\sum_{j=1}^{r} \frac{b M_{j}}{2}+\sum_{j=r+1}^{s} \frac{M c_{j}}{2} .
\end{gathered}
$$


Here we use that

$$
a_{(i+1)+M / 2+M_{j}^{\prime}}^{j}=a_{\left(M_{j}^{\prime}+M+1\right)-(M / 2-i)}^{j}=-(M / 2-i) c_{j}
$$

for $0 \leq i \leq M / 2-1$, keeping in mind that in this case the roles of the $b$ 's and $c$ 's are reversed.

$$
\begin{gathered}
Y_{i+1}: 1 \leq i \leq M / 2-1:-b\left(C_{i}^{2}\right)_{Y_{i+1}}+\sum_{j=1}^{r} a_{i+1}^{j}-\sum_{j=r+1}^{s} a_{(i+1)+M / 2+M_{j}^{\prime}}^{j} \\
=-i b C^{2}-\sum_{j=1}^{r} \frac{M_{j} b}{2}-\sum_{j=1}^{r} i c_{j}+\sum_{j=r+1}^{s}(M / 2-i) c_{j} \\
=-i b C^{2}-\sum_{j=1}^{s} i c_{j}-\sum_{j=1}^{r} \frac{M_{j} b}{2}+\sum_{j=r+1}^{s} \frac{M c_{j}}{2} . \\
Y_{M / 2+1}:-b\left(C_{M / 2}^{2}\right)_{Y_{M / 2+1}}+\sum_{j=1}^{r} a_{M / 2+1}^{j}-\sum_{j=r+1}^{s} a_{1}^{j} \\
=-\frac{b M}{2} C^{2}-\sum_{j=1}^{r} \frac{M_{j} b}{2}-\sum_{j=1}^{r} \frac{M c_{j}}{2} \\
=-\frac{b M}{2} C^{2}-\sum_{j=1}^{s} \frac{M c_{j}}{2}-\sum_{j=1}^{r} \frac{M_{j} b}{2}+\sum_{j=r+1}^{s} \frac{M c_{j}}{2} .
\end{gathered}
$$

Now we use

$$
a_{(i+1)-M / 2+M_{j}^{\prime}}^{j}=a_{\left(M+M_{j}^{\prime}+1\right)-(3 M / 2-i)}^{j}=-(3 M / 2-i) c_{j}
$$

for $M / 2+1 \leq i \leq M-1$.

$$
\begin{aligned}
Y_{i+1} & : M / 2+1 \leq i \leq M-1: \\
& -b\left(C_{i}^{2}\right)_{Y_{i+1}}+\sum_{j=1}^{r} a_{i+1}^{j}-\sum_{j=r+1}^{s} a_{(i+1)-M / 2+M_{j}^{\prime}}^{j} \\
& =-i b C^{2}-\sum_{j=1}^{r} \frac{M_{j} b}{2}-\sum_{j=r+1}^{s} M_{j} b-\sum_{j=1}^{r} i c_{j}+\sum_{j=r+1}^{s}(3 M / 2-i) c_{j} \\
& =-i b C^{2}-\sum_{j=1}^{s} i c_{j}-\sum_{j=1}^{r} \frac{M_{j} b}{2}+\sum_{j=r+1}^{s} \frac{M c_{j}}{2}
\end{aligned}
$$

using the fact that $M_{j} b=M c_{j}$. Again, with $\alpha=-b C^{2}-\sum_{j=1}^{s} c_{j}$, we get, after choosing a different representative by adding $\alpha+\sum_{j=1}^{r} M_{j} b / 2-\sum_{j=r+1}^{s} M c_{j} / 2$ to each component,

$$
Y_{i}: i \alpha
$$

Proof of Theorem 3.6. First the case that $M$ is odd.

We follow the same procedure and notation as in Theorem 3.4. The only additional complication is to take into account what the image of $\gamma$ is in $H^{0}(\mathcal{F})$ on the 
curves over the collision points of type $I_{M}+I_{N_{i}}^{*}$. But this is given by Theorem 3.5. For each collision $I_{M}+I_{N_{i}}^{*}$, set

$$
\alpha_{j}^{i}= \begin{cases}\left(\phi_{1}(b)\right)_{j}, & M \text { odd } \\ \left(\phi_{1}\left(b, d_{i}\right)\right)_{j}, & M, N_{i} \text { even }, \\ \left(\phi_{1}\left(b_{i}\right)\right)_{j}, & M \text { even, } N_{i} \text { odd }\end{cases}
$$

using the notation in the statement of Theorem 3.5. If $f_{1}^{\prime i}, \ldots, f_{m}^{\prime i}$ are the components of the fibre over the collision point, then a representative of $\beta(\gamma)$ in the components $\bigoplus_{j} H^{0}\left(f_{j}^{\prime i}, \mathbf{Q} / \mathbf{Z}\right)$ of $H^{0}(\mathcal{F})$ comes from specialising the $\alpha_{i}$ 's, as in the proof of Theorem 3.5 using the last part of Table 2.

On $Y_{1}$, using instead the section $\sigma_{0} \cap Y_{1}$ (the section $C_{0}$ in Figure A.3), we get a contribution of

$$
Y_{1}: \sum_{j=1}^{r} a_{M^{\prime}+1+M_{j} / 2}^{j}+\sum_{j=1}^{u} \alpha_{1}^{j}=\sum_{j=1}^{r}-\frac{M_{j} b}{2} .
$$

On $Y_{i+1}, 1 \leq i \leq(M-1) / 2$, we use the section $C_{i}$, keeping in mind that $C_{i}$ now intersects both the curves $f_{i+1}^{j}$ and $f_{M^{\prime}+1-i}^{j}$ :

$$
\begin{aligned}
Y_{i+1}, 1 & \leq i \leq(M-1) / 2: \\
& -b\left(C_{i}^{2}\right)_{Y_{i+1}}+\sum_{j=1}^{r} a_{i+1}^{j}+\sum_{j=1}^{r} a_{M^{\prime}+1-i}^{j}+\sum_{j=1}^{u} \alpha_{n_{1}}^{j} \\
& =-2 i b C^{2}+2 b u-\sum_{j=1}^{r} M_{j} b-\sum_{j=1}^{u} \eta_{j} b-\sum_{j=1}^{r} i c_{j}-\sum_{j=1}^{r}(M-i) c_{j} \\
& =-\sum_{j=1}^{r} 2 M_{j} b \\
& =0
\end{aligned}
$$

keeping in mind that $b=0$ or $1 / 2$, that the $\eta_{j}$ 's are even, $(M>1$ in this range), and $M c_{j}=M_{j} b$. (Remember this is all in $\mathbf{Q} / \mathbf{Z}$, where $2 b=0$.)

For $Y_{(M+3) / 2}$, the contribution is calculated as follows. On $Y_{(M+3) / 2}$,

$$
C_{(M+1) / 2} \equiv 2 C_{0}+a f
$$

for some integer $a$, with $C_{0}$ a section of $Y_{(M+3) / 2} \rightarrow C$, and since $Y_{(M+3) / 2} \cong \mathbf{P}_{C}(\mathcal{E})$ with $c_{1}(\mathcal{E})=C^{2}-u / 2($ Theorem 3.2$), C_{0}^{2}$ must have the same parity as $C^{2}-u / 2$. Now

$$
\left(C_{(M+1) / 2}^{2}\right)_{Y_{(M+3) / 2}}=4 C_{0}^{2}+4 a=2 u,
$$

so $C_{0}^{2}+a \equiv u / 2 \quad(\bmod 2)$, and hence $a$ has the same parity as $C^{2}$ since $u$ is even. Since $b$ is 0 or $1 / 2$, the class of $-b C_{(M+1) / 2}$ in $H^{2}\left(Y_{(M+3) / 2}, \mathbf{Q} / \mathbf{Z}\right)$ is $b C^{2} f$, where 
$f$ is a fibre of $Y_{(M+3) / 2} \rightarrow C$. Thus we get, in total,

$$
\begin{aligned}
Y_{(M+3) / 2}: & b C^{2}+\sum_{j=1}^{r} a_{(M+3) / 2}^{j}+\sum_{j=1}^{u} \alpha_{N_{j}+4}^{j} \\
= & b C^{2}-\sum_{j=1}^{r} M c_{j}+\left(\# N_{j} \text { even }\right) b \\
= & b C^{2}+\left(\# N_{j} \text { even }\right) b,
\end{aligned}
$$

since $M c_{j}=M_{j} b$ and the $M_{j}$ 's are all even. Notice that we have

$$
C^{2} \equiv M C^{2} \equiv \sum_{j=1}^{r} M_{j}+\sum_{j=1}^{u} N_{j} \equiv \# N_{j} \text { odd }(\bmod 2)
$$

so we get for $Y_{(M+3) / 2}$

$$
b C^{2}+\left(\# N_{j} \text { even }\right) b=\left(\# N_{j} \text { odd }\right) b+\left(\# N_{j} \text { even }\right) b=u b=0 .
$$

Now $H^{3}\left(S, \mathcal{E}_{t}\right)=\operatorname{coker}\left(\mathbf{Q} / \mathbf{Z} \rightarrow(\mathbf{Q} / \mathbf{Z})^{(M+3) / 2}\right)$ and the latter map is given by $a \mapsto(a, 2 a, \ldots, 2 a)$, so we can adjust our representative for $\phi_{1}(\gamma) \in H^{3}\left(S, \mathcal{E}_{t}\right)$ by $\left(\sum_{j=1}^{r} \frac{M_{j} b}{2}, 0, \ldots, 0\right)$. Thus we obtain

$$
Y_{i}, 1 \leq i \leq(M+3) / 2: 0 .
$$

Next, the case that $M$ is even, following the same procedure:

$$
\begin{aligned}
& Y_{1}: \sum_{j=1}^{r} a_{M+1+M_{j} / 2}-\sum_{j=r+1}^{s} a_{1+M / 2+M_{j}^{\prime}}+\sum_{j=1}^{u} \alpha_{1}^{j} \\
& =-\sum_{j=1}^{r} \frac{M_{j} b}{2}+\sum_{j=r+1}^{s} \frac{M c_{j}}{2} . \\
& Y_{i+1}, 1 \leq i \leq M / 2-1:-b\left(C_{i}^{2}\right)_{Y_{i+1}}+\sum_{j=1}^{r} a_{i+1}^{j}+\sum_{j=1}^{r} a_{M+1-i}^{j} \\
& -\sum_{j=r+1}^{s} a_{(i+1)+M / 2+M_{j}^{\prime}}^{j}-\sum_{j=r+1}^{s} a_{M / 2-i+1+M_{j}^{\prime}}^{j}+\sum_{j=1}^{u} \alpha_{n_{1}}^{j} \\
& =-2 i b C^{2}+2 b u-\sum_{j=1}^{r} b M_{j}-\sum_{j=1}^{u} b \eta_{j}-\sum_{j=1}^{r} i c_{j}-\sum_{j=1}^{r}(M-i) c_{j} \\
& +\sum_{j=r+1}^{s}(M / 2-i) c_{j}+\sum_{j=r+1}^{s}(M / 2+i) c_{j} \\
& =-\sum_{j=1}^{r} 2 b M_{j}+\sum_{j=r+1}^{s} M c_{j} \\
& =\sum_{j=r+1}^{s} M c_{j}
\end{aligned}
$$


For $Y_{M / 2+1}$, we use the section $\sigma^{\prime}$ where $\sigma^{\prime}$ is any section of $k: Y_{M / 2+1} \rightarrow C$ intersecting the reducible fibres in the $Y_{N_{i}+5}^{\prime} \cap Y_{M / 2+1}$ component at the $I_{M}+I_{N_{i}}^{*}$ collisions and the component $f_{1}^{j}$ at the $I_{M}+I_{M_{j}}$ collisions, $r+1 \leq j \leq s$. This gives us

$$
\begin{aligned}
Y_{M / 2+1}: & -b C_{M / 2} \cdot \sigma^{\prime}+\sum_{j=1}^{r} a_{M / 2+1}^{j}-\sum_{j=r+1}^{s} a_{1}^{j}+\sum_{j=1}^{u} \alpha_{N_{j}+5}^{j} \\
& =-b C_{M / 2} \cdot \sigma^{\prime}-\sum_{j=1}^{r} \frac{M c_{j}}{2}+\sum_{j=1}^{u} \alpha^{j} .
\end{aligned}
$$

Choosing the appropriate representative by subtracting

$$
-\sum_{j=1}^{r} \frac{M_{j} b}{2}+\sum_{j=r+1}^{s} \frac{M c_{j}}{2}
$$

from the values for $Y_{1}$ and $Y_{M / 2+1}$ and twice that for the others, keeping in mind $M_{j}$ is even for $1 \leq j \leq r$, we get the desired results for $Y_{i}, i \leq M / 2$. For $Y_{M / 2+1}$, we have

$$
\begin{aligned}
-b C_{M / 2} \cdot \sigma^{\prime}-\sum_{j=r+1}^{s} \frac{M c_{j}}{2}+\sum_{j=1}^{u} \alpha^{j} & =-b C_{M / 2} \cdot \sigma^{\prime}+\sum_{j=1}^{r} \frac{M_{j} b}{2}-\sum_{j=1}^{s} \frac{M c_{j}}{2}+\sum_{j=1}^{u} \alpha^{j} \\
& =-b\left(C_{M / 2} \cdot \sigma^{\prime}+\sum_{j=1}^{r} \frac{M_{j}}{2}\right)-\sum_{j=1}^{s} \frac{M c_{j}}{2}+\sum_{j=1}^{u} \alpha^{j} .
\end{aligned}
$$

If we remember that $M_{j}$ is even for $1 \leq j \leq r$, then $a=C_{M / 2} \cdot \sigma^{\prime}+\sum_{j=1}^{r} \frac{M_{j}}{2}$ is the desired integer. 


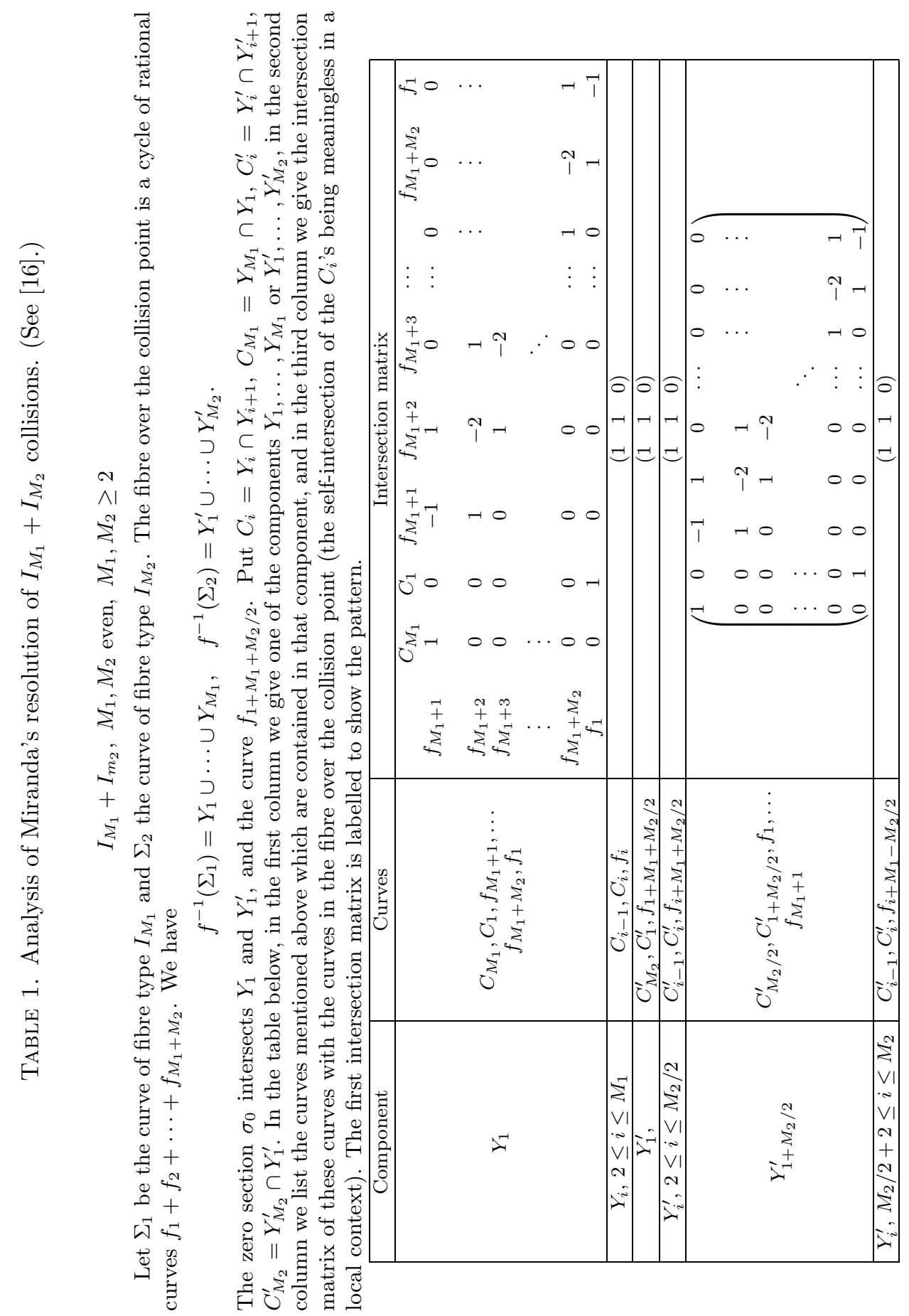




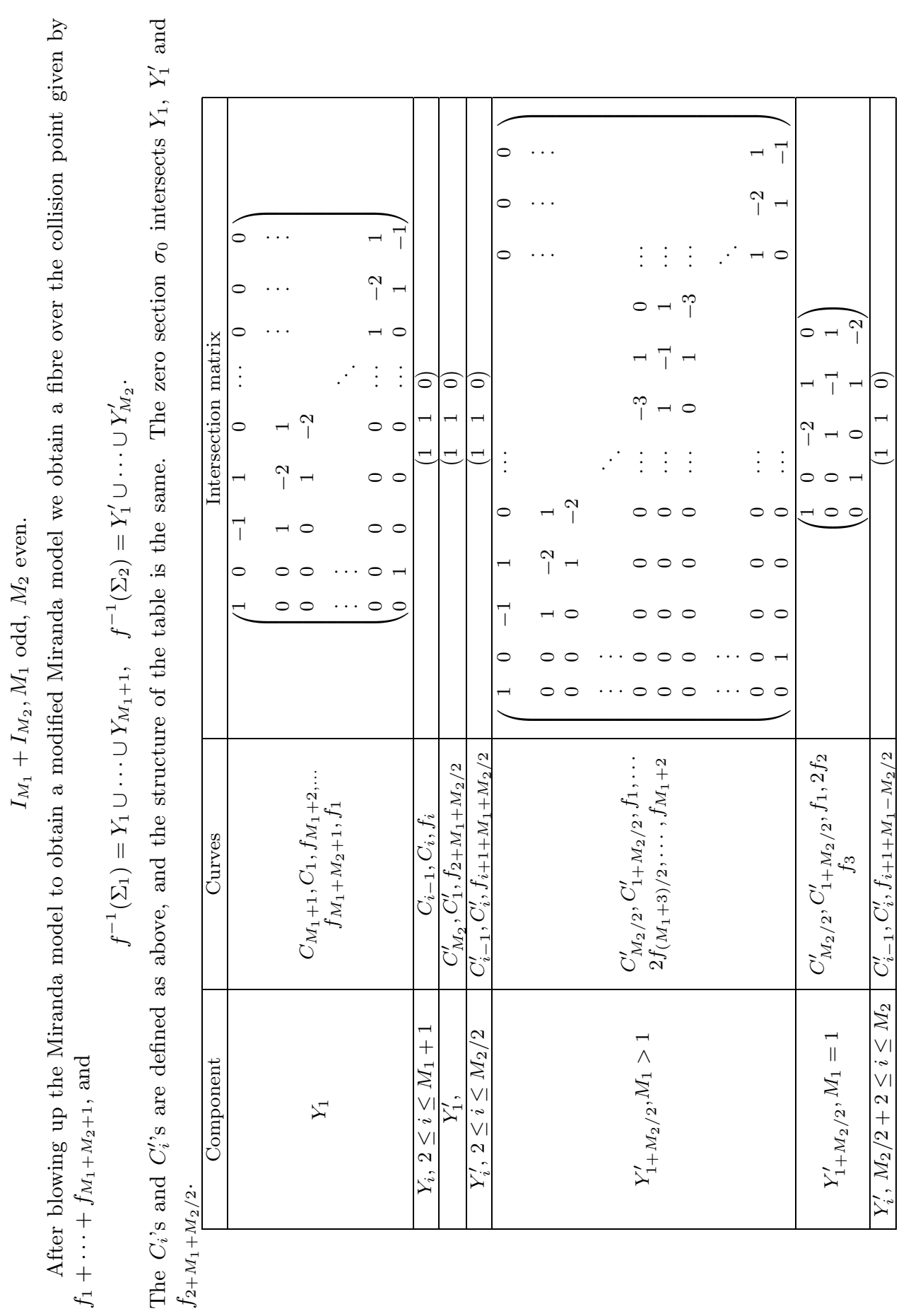


TABLE 2. The analysis of Miranda's resolution of $I_{M_{1}}+I_{M_{2}}^{*}$ collisions. (See [16].)

Here, we have $\Sigma_{1}$ a curve of fibre type $I_{M_{1}}$ and $\Sigma_{2}$ a curve of fibre type $I_{M_{2}}^{*}$. The fibre over the collision point is

$$
\begin{gathered}
\begin{cases}f_{1}+f_{2}+2 f_{3}+\cdots+2 f_{M-2}+f_{M-1}+f_{M}, & M_{1} \text { even, } M=M_{2}+M_{1} / 2+5, \\
f_{1}+f_{2}+2 f_{3}+\cdots+2 f_{M}, & M_{1} \text { odd }, M=M_{2}+\left(M_{1}-1\right) / 2+5 .\end{cases} \\
f^{-1}\left(\Sigma_{1}\right)=Y_{1} \cup \cdots \cup Y_{m_{1}}, m_{1}= \begin{cases}M_{1} / 2+1, & M_{1} \text { even, } \\
\left(M_{1}+3\right) / 2, & M_{1} \text { odd. }\end{cases} \\
f^{-1}\left(\Sigma_{2}\right)=Y_{1}^{\prime} \cup \cdots \cup Y_{m_{2}}^{\prime}, m_{2}= \begin{cases}M_{2}+5, & M_{1} \text { even, } \\
M_{2}+4, & M_{1} \text { odd. }\end{cases}
\end{gathered}
$$

Set

$$
\begin{gathered}
n_{1}= \begin{cases}M_{2}+3 & \text { if } M_{2} \text { is even; } \\
M_{2}+2 & \text { if } M_{2} \text { is odd. }\end{cases} \\
C_{i}=Y_{i} \cap Y_{i+1}
\end{gathered}
$$

The zero section $\sigma_{0}$ intersects $Y_{1}, Y_{1}^{\prime}$ and $f_{1}$. $Y_{1}$ and $Y_{m_{1}}$ are ruled over $\Sigma_{1} ; Y_{i}$, $2 \leq i \leq m_{1}-1$ are ruled over a double cover of $\Sigma_{1}$, branched at the collision point. $Y_{1}^{\prime}, \ldots, Y_{m_{2}-1}^{\prime}$ are ruled over $\Sigma_{2}$, as well as $Y_{m_{2}}^{\prime}$ unless $M_{1}$ is odd, in which case $Y_{m_{2}}^{\prime}$ is ruled over a double cover of $\Sigma_{2}$ branched at the collision point.

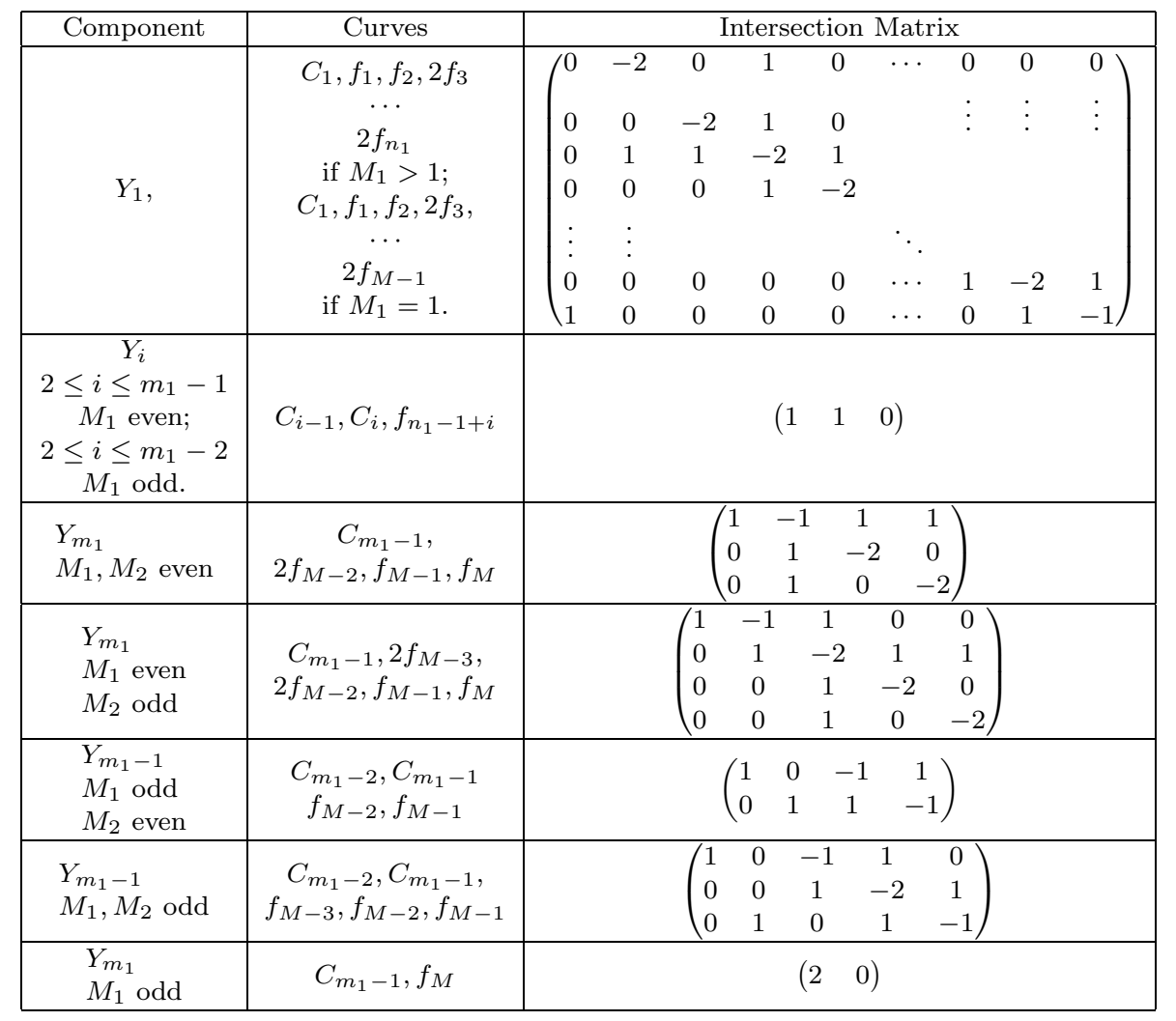


TABLE 2. continued

\begin{tabular}{|c|c|}
\hline Component & Curves \\
\hline$Y_{i}^{\prime}, i=1,2$ & $f_{i}$ \\
\hline $\begin{array}{c}Y_{i}^{\prime} \\
3 \leq i \leq n_{1}-1 \\
\end{array}$ & $f_{i}$ \\
\hline $\begin{array}{l}Y_{n_{1}}^{\prime} \\
M_{2} \text { even }\end{array}$ & $f_{n_{1}}, \ldots, f_{M-2}$ \\
\hline $\begin{array}{l}Y_{n_{1}}^{\prime} \\
M_{2} \text { odd }\end{array}$ & $f_{n_{1}}, \ldots, f_{M-3}$ \\
\hline $\begin{array}{c}Y_{i}^{\prime} \\
i=m_{2}-1, m_{2} \\
M_{1} \text { even }\end{array}$ & $\begin{array}{c}f_{M-1} \\
\text { or } \\
f_{M}\end{array}$ \\
\hline $\begin{array}{c}Y_{M_{2}+3}^{\prime} \\
M_{2} \text { odd }\end{array}$ & $f_{M-2}$ \\
\hline $\begin{array}{l}Y_{M_{2}+4}^{\prime} \\
M_{1} \text { odd }\end{array}$ & $f_{M-1}, f_{M}$ \\
\hline
\end{tabular}

\section{REFERENCES}

1. Barth, W., Peters, C., and Van de Ven, A., Compact Complex Surfaces, (Ergeb. Math. Grenzbeg. 3. Folge, vol. 4), Springer, 1984. MR 86c:32026

2. Bloch, S., Torsion Algebraic Cycles and a Theorem of Roitman, Compositio Math. 39 (1979), 107-127. MR 80k:14012

3. Bloch, S., and Ogus, A., Gersten's Conjecture and the Homology of Schemes, Ann. Éc. Norm. Sup. 7 (1974), 181-202. MR 54:318

4. Cossec, F., and Dolgachev, I., Enriques Surfaces I, Birkhäuser, Boston, 1989. MR 90h:14052

5. Colliot-Thélène, J.L., Sansuc, J.J., and Soulé, C., Torsion dans le Groupe de Chow de Codimension Deux, Duke Math. J. 50 (1983), 763-801. MR 85d:14010

6. Cox, D., and Zucker, S., Intersection Numbers of Sections of Elliptic Surfaces, Inventiones Math. 53 (1979), 1-44. MR 81i:14023

7. Deligne, P., Courbes Elliptiques: Formulaire d'après J. Tate, Modular Functions in One Variable IV, Lecture Notes in Mathematics, vol. 476, Springer-Verlag, 1975, pp. 53-74. MR 52:8135

8. Dolgachev, I., and Gross, M., Elliptic Three-folds I: Ogg-Shafarevich Theory, J. Algebraic Geometry 3 (1994), 39-80. MR 95d:14037

9. Fujimoto, Y., Logarithmic Transformations on Elliptic Fibre Spaces, J. Math. Kyoto Univ. 28 (1988), 91-110. MR 88m:32053

10. Fulton, W., Intersection Theory, (Ergeb. Math. Grenzgeb., 3. Folge, vol. 2), Berlin, Heidelberg, New York, Springer, 1984. MR 85k:14004

11. Grassi, A., Minimal Models of Elliptic Threefolds, Ph.D. Thesis, Duke University, 1990.

12. Gross, M., A Finiteness Theorem for Elliptic Calabi-Yau Threefolds, Duke Math. J. 74 (1994), 271-299. MR 95c: 14047

13. Grothendieck, A., Le Groupe de Brauer, I, II, III, Dix Exposés sur Cohomologie des Schémas, North-Holland, Amsterdam, 1968, pp. 46-188. MR 39:5586a,b,c

14. Kodaira, K., On Compact Complex Analytic Surfaces, I, Ann. Math. 71 (1960), 111-152; II, Ann. Math 77 (1963), 563-626; III, Ann. Math. 78 (1963), 1-40. MR 24:A2396; MR 32: 1730

15. Milne, J., Étale Cohomology, Princeton Univ. Press, 1980. MR 81j:14002

16. Miranda, R., Smooth Models for Elliptic Threefolds, Birational Geometry of Degenerations, Birkhäuser, 1983, pp. 85-133. MR 84f:14024

17. Mumford, D., and Suominen, K., Introduction to the Theory of Moduli, Algebraic Geometry, Oslo 1970, Wolters-Noordhoff Press, 1972, pp. 171-222. MR 55:10455

18. Nakayama, N., On Weierstrass Models, Algebraic Geometry and Commutative Algebra in Honor of Masayoshi Nagata (1987), 405-431. MR 90m:14030 
19. Nakayama, N., Local Structure of an Elliptic Fibration, Preprint, Univ. of Tokyo, 1991.

20. Ogg, A., Cohomology of Abelian Varieties over Function Fields, Ann. Math. 76 (1962), 185212. MR 27:5758

21. Shafarevich, I., Principal Homogeneous Spaces over Function Fields, AMS Translations 37 (1964), 85-113. MR 29:110 (Russian original)

Department of Mathematics, Cornell University, Ithaca, New York 14853

E-mail address: mgross@math.cornell.edu 